\title{
COMPLETE SYMPLECTIC QUADRICS AND KONTSEVICH SPACES OF CONICS IN LAGRANGIAN GRASSMANNIANS
}

\author{
ELSA CORNIANI AND ALEX MASSARENTI
}

\begin{abstract}
A wonderful compactification of an orbit under the action of a semi-simple and simply connected group is a smooth projective variety containing the orbit as a dense open subset, and where the added boundary divisor is simple normal crossing. We construct the wonderful compactification of the space of symmetric and symplectic matrices, and investigate its geometry. As an application, we describe the birational geometry of the Kontsevich spaces parametrizing conics in Lagrangian Grassmannians.
\end{abstract}

\section{INTRODUCTION}

The wonderful compactification of a symmetric space was introduced by C. De Concini and C. Procesi in DCP83]. Later on, D. Luna gave a more general definition of wonderful variety and then he proved that, according to his definition, all wonderful varieties are spherical [Lun96].

Let $\mathscr{G}$ be a reductive group, and $\mathscr{B} \subset \mathscr{G}$ a Borel subgroup. A spherical variety is a variety admitting an action of $\mathscr{G}$ with an open dense $\mathscr{B}$-orbit. For wonderful varieties we require in addition the existence of an open orbit whose complementary set is a simple normal crossing divisor $D_{1} \cup \cdots \cup D_{r}$, where the $D_{i}$ are the $\mathscr{G}$-invariant prime divisors in $X$. The number $r$ is called the rank of $X$. Note that $\mathscr{G}$ has $2^{r}$ orbits in $X$ given by all the possible intersections among the $D_{i}$. The unique closed orbit is $\bigcap_{i=1}^{r} D_{i}$.

Apart from their role in group theory, wonderful varieties proved themselves important in enumerative geometry and recently also in birational geometry. We refer to [BL11, Per14, Pez18] for comprehensive treatments of these topics.

Classical examples of wonderful varieties are the spaces of complete quadrics and of complete collineations. These spaces have been studied both from the geometrical and enumerative point of view [Sem48], [Sem51, [Sem52, [Tyr56, [Vai82], [Vai84], [KT88], [LLT89], [Tha99]. An aspect that will be fundamental in this paper is that spaces of complete quadrics and collineations play a role in the study of other moduli spaces such as Hilbert schemes and Kontsevich spaces of stable maps [Alg56, Pie82, Cav16]. The birational geometry of the spaces of complete quadrics and collineations, mostly from the point of view of Mori theory, has recently been studied in Hue15, Mas20a, Mas20b.

The spaces of complete collineations and quadrics have been constructed, as a sequence of blow-ups, by I. Vainsencher in [Vai84, Vai82], and a similar construction for complete skew-forms has been carried out by M. Thaddeus in [Tha99]. In this paper we construct the wonderful compactification of the space of symmetric and symplectic matrices. More precisely, we summarize our main results in Propositions 3.6] 3.13, and Theorem [3.19 as follows:

Theorem 1.1. Let $\mathbb{P}^{N}$ be the projective space parametrizing $2 r \times 2 r$ symmetric matrices modulo scalar, consider the following $S p(2 r)$-action:

$$
\begin{array}{ccc}
S p(2 r) \times \mathbb{P}^{N} & \longrightarrow & \mathbb{P}^{N} \\
(M, Z) & \longmapsto & M Z M^{t}
\end{array}
$$

and denote by $X_{2 r} \subset \mathbb{P}^{N}$ the closure of the $S p(2 r)$-orbit of the identity. Then $X_{2 r}$ admits a stratification

$$
Y_{1} \subset Y_{2} \subset \ldots Y_{r} \subset X_{2 r}
$$

where the variety $Y_{k}$ parametrizes matrices in $X_{2 r}$ of rank at most $k, \operatorname{dim}\left(Y_{k}\right)=2 r k+k-k^{2}-1$ for $k=1, \ldots, r$, and $\operatorname{dim}\left(X_{2 r}\right)=r(r+1)$.

Furthermore, consider the following sequence of blow-ups

$$
\mathcal{S}_{2 r}:=X_{2 r}^{(r-1)} \rightarrow X_{2 r}^{(r-2)} \rightarrow X_{2 r}^{(r-3)} \rightarrow \cdots \rightarrow X_{2 r}^{(1)} \rightarrow X_{2 r}^{(0)}:=X_{2 r}
$$

Date: November 5, 2021.

2010 Mathematics Subject Classification. Primary 14M27, 14E30; Secondary 14J45, 14N05, 14E07.

Key words and phrases. Wonderful compactifications; Mori dream spaces; Cox rings; Spherical varieties; Stable maps. 
where $X_{2 r}^{(k)} \rightarrow X_{2 r}^{(k-1)}$ is the blow-up of the strict transform of $Y_{k}$ in $X_{2 r}^{(k-1)}$ for $k=1, \ldots, r-1$. Denote by $E_{k} \subset \mathcal{S}_{2 r}$ the exceptional divisor over $Y_{k}$ for $k=1, \ldots, r-1$, and by $S_{r}^{(r-1)}\left(\mathcal{V}_{2}^{2 r-1}\right)$ the strict transform of the divisor $Y_{r} \subset X_{2 r}$. Then $E_{1}, \ldots, E_{r-1}, S_{r}^{(r-1)}\left(\mathcal{V}_{2}^{2 r-1}\right)$ are smooth and intersect transversally. Furthermore, the closures of the orbits of the $S p(2 r)$-action on $\mathcal{S}_{2 r}$ induced by the $S p(2 r)$-action above are given by all the possible intersections among $E_{1}, \ldots, E_{r-1}, S_{r}^{(r-1)}\left(\mathcal{V}_{2}^{2 r-1}\right)$ and $\mathcal{S}_{2 r}$ itself. Therefore $\mathcal{S}_{2 r}$ is wonderful.

We will call $\mathcal{S}_{2 r}$ the space of complete symplectic quadrics of dimension $2 r-2$. By Proposition $3.13 Y_{k}$ is the intersection of $X_{2 r}$ with the secant variety $\operatorname{Sec}_{k}\left(\mathcal{V}_{2}^{2 r-1}\right)$ that is the closure of the union of the $(k-1)$-planes generated by $k$ general points on the Veronese variety $\mathcal{V}_{2}^{2 r-1}$ of degree two and dimension $2 r-1$.

Note that the formula for the dimension of $Y_{k}$ in Theorem 1.1 yields that $\mathcal{V}_{2}^{2 r-1}$ is entirely contained in $X_{2 r}$, while for $r \geqslant 2$ the orbit closure $X_{2 r}$ intersects $\operatorname{Sec}_{k}\left(\mathcal{V}_{2}^{2 r-1}\right)$ in a proper subvariety. Furthermore, by Proposition 3.15 we have that set-theoretically $\operatorname{Sec}_{k}\left(\mathcal{V}_{2}^{2 r-1}\right) \cap X_{2 r}=\operatorname{Sec}_{r}\left(\mathcal{V}_{2}^{2 r-1}\right) \cap X_{2 r}$ for $k \geqslant r$. Interestingly, this means that if $M$ is a symmetric $2 r \times 2 r$ matrix that is a limit of a family of symplectic matrices then either $1 \leqslant \operatorname{rank}(M) \leqslant r$ or $\operatorname{rank}(M)=2 r$.

For instance, by Proposition $3.16 X_{4}$ is the Grassmannian $\mathbb{G}(1,4)$ of lines in $\mathbb{P}^{4}$. In this case by Theorem 1.1 we have that $\mathcal{S}_{4}$ is the blow-up of $\mathbb{G}(1,4)$ along the Veronese 3 -fold $\mathcal{V}_{2}^{3} \subset \mathbb{G}(1,4)$. This is a wonderful variety of rank two. As remarked in Was96 wonderful varieties of rank two are a building block in the theory of spherical varieties. The wonderful compactification $\mathcal{S}_{4}$ is the sixth variety in [Was96. Table C], and will be a central character throughout the whole paper.

Remark 1.2. The use of wonderful compactifications in enumerative geometry dates back to the solution of M. Chasles to a problem posed by J. Steiner asking how many conics in the plane are tangent to five given general conics Kle80]. Steiner's answer, which then turned out to be wrong, was $6^{5}=7776$. Later on Chasles computed the right number which is 3264 .

Although enumerative problems are not within the scope of this paper, we give a simple application of our construction in enumerative geometry. It is well known that there are 92 quadric surfaces in $\mathbb{P}^{3}$ that are tangent to nine general lines BFS20, Remark 4.3]. The points of $\mathcal{S}_{4}$ in a divisor of class $2 H-E_{1}$, where $H$ is the pull-back of the hyperplane class of $X_{4}$, correspond to the symplectic quadrics in $\mathbb{P}^{3}$ that are tangent to a general line. We have that $\left(2 H-E_{1}\right)^{6}=40$. From the enumerative point of view this means that there are exactly 40 symplectic quadrics in $\mathbb{P}^{3}$ that are tangent to six general lines.

The variety $X_{2 r}$ is singular for $r \geqslant 3$. The wonderful variety $\mathcal{S}_{2 r}$ may be seen as an incarnation, in the singular setting, of the process producing a wonderful compactification from a conical one in MP98. Furthermore, by Proposition $3.18 \mathcal{S}_{2 r}$ provides a resolution of a variety with conical singularities as remarked in [MP98, Section 3.3].

In Section 4 and 5 we take advantage of the spherical structure of $\mathcal{S}_{2 r}$ to study its birational geometry from the point of view of Mori theory. Roughly speaking, a Mori dream space is a projective variety $X$ whose cone of effective divisors $\operatorname{Eff}(X)$ admits a well-behaved decomposition into convex sets, called Mori chamber decomposition, and these chambers are the nef cones of birational models of $X$. These varieties, introduced by $\mathrm{Y}$. Hu and S. Keel in [HK00, are named so because they behave in the best possible way from the point of view of the minimal model program. In general, to determine whether or not a variety is a Mori dream space, and in case to study in detail its Mori chamber decomposition is a hard problem. This has been done for instance when $X$ is obtained by blowing-up points in a projective space [Muk01, [CT06], AM16, [AC17, [BM21], [LP17].

Spherical varieties are Mori dream spaces, we refer to Per14 for a comprehensive treatment of these topics. Cox rings were first introduced by D. A. Cox for toric varieties Cox95, and then his construction was generalized to projective varieties in [HK00]. These algebraic objects are basically universal homogeneous coordinate rings of projective varieties, defined as the direct sum of the spaces of sections of all isomorphism classes of line bundles on them. We have that a normal $\mathbb{Q}$-factorial projective variety $X$, over an algebraically closed field, with finitely generated Picard group is a Mori dream space if and only if its Cox ring is finitely generated [HK00, Proposition 2.9]. Summing-up the results in Propositions 4.5, 4.7, 5.10 and Theorem 5.12 we have the following:

Theorem 1.3. Fix homogeneous coordinates $\left[z_{0,0}: \cdots: z_{n, n}\right]$ on $\mathbb{P}^{N}$, and consider the blow-up $f: \mathcal{S}_{2 r} \rightarrow X_{2 r} \subset \mathbb{P}^{N}$ with exceptional divisors $E_{1}, \ldots, E_{r-1}$ in Theorem 1.1. For $i=1, \ldots, r$ we define the divisors $D_{i}$ as the strict transforms in $\mathcal{S}_{2 r}$ of the divisor given by the intersection of

$$
\operatorname{det}\left(\begin{array}{ccc}
z_{0,0} & \cdots & z_{0, i-1} \\
\vdots & \ddots & \vdots \\
z_{0, i-1} & \cdots & z_{i-1, i-1}
\end{array}\right)=0
$$


with $X_{2 r}$, and let $H$ be the pull-back of the hyperplane section of $X_{2 r} \subset \mathbb{P}^{N}$ to $\mathcal{S}_{2 r}$.

The Picard rank of $\mathcal{S}_{2 r}$ is $\rho\left(\mathcal{S}_{2 r}\right)=r$ and $\operatorname{Pic}\left(\mathcal{S}_{2 r}\right)$ is generated by $H, E_{1}, \ldots, E_{r-1}$. Furthermore, the effective cone $\operatorname{Eff}\left(\mathcal{S}_{2 r}\right)$ is generated by $E_{1}, \ldots, E_{r-1}, S_{r}^{(r-1)}\left(\mathcal{V}_{2}^{2 r-1}\right)$, the nef cone $\operatorname{Nef}\left(\mathcal{S}_{2 r}\right)$ is generated by $D_{1}, \ldots, D_{r}$, and the Cox ring of $\mathcal{S}_{2 r}$ is generated by the sections of $E_{1}, \ldots, E_{r-1}, S_{r}^{(r-1)}\left(\mathcal{V}_{2}^{2 r-1}\right), D_{1}, \ldots, D_{r}$.

Finally, the Mori chamber decomposition of the $\operatorname{Eff}\left(\mathcal{S}_{4}\right)$ has three chambers, and the Mori chamber decomposition of the $\operatorname{Eff}\left(\mathcal{S}_{6}\right)$ has nine chambers.

We refer to Proposition 5.10 and Theorem 5.12 for a detailed description of the Mori chamber decompositions.

In Section 6 we investigate the birational geometry of Kontsevich moduli spaces of conics in Lagrangian Grassmannians. These spaces are denoted by $\bar{M}_{g, n}(X, \beta)$ where $X$ is a projective scheme and $\beta \in H_{2}(X, \mathbb{Z})$ is the homology class of a curve in $X$. A point in $\bar{M}_{g, n}(X, \beta)$ corresponds to a holomorphic map $\alpha$ from an $n$-pointed genus $g$ curve $C$ to $X$ such that $\alpha_{*}([C])=\beta$. If $X$ is a homogeneous variety then there exists a smooth, irreducible Deligne-Mumford stack $\overline{\mathcal{M}}_{0, n}(X, \beta)$ whose coarse moduli space is $\bar{M}_{0, n}(X, \beta)$ [FP97]. When $X$ is a Lagrangian Grassmannian the class $\beta$ is then completely determined by its degree and we will write $\beta=d[L]$, where $[L]$ is the class of a line in the Plücker embedding. The Mori theory of the spaces $\bar{M}_{0, n}(X, \beta)$, especially when the target variety is a projective space or a Grassmannian, has been widely investigated in a series of papers [CS06], Che08], CHS08, CHS09, CC10, CC11, CM17.

On the Kontsevich space $\bar{M}_{0,0}(L G(r, 2 r), 2)$ of conics in the Lagrangian Grassmannian, $L G(r, 2 r)$ parametrizing Lagrangian subspaces of a $2 r$-dimensional symplectic vector space, we consider the divisor classes: $\Delta^{r}$ of maps with reducible domain, $T^{r}$ of conics tangent to a fixed hyperplane section of $L G(r, 2 r), H_{\sigma_{2}}^{r}$ of conics intersecting a fixed codimension two Schubert variety $\Sigma_{2}^{r} \subset L G(r, 2 r)$, and $D_{u n b}^{r}$ which we now define. A stable map $\alpha: \mathbb{P}^{1} \rightarrow$ $L G(r, 2 r)$ induces a rank two subbundle $\mathcal{E}_{\alpha} \subset \mathcal{O}_{\mathbb{P}^{1}} \otimes K^{2 r}$. If $r=2$ we define $D_{\text {unb }}$ as the closure of the locus of maps $\left[\mathbb{P}^{1}, \alpha\right] \in \bar{M}_{0,0}(L G(2,4), 2)$ such that $\mathcal{E}_{\alpha} \neq \mathcal{O}_{\mathbb{P}^{1}}(-1)^{\oplus 2}$. If $r \geqslant 3$ there is a trivial subbundle $\mathcal{O}_{\mathbb{P}^{1}}^{\oplus r-2} \subset \mathcal{E}_{\alpha}$ which induces a $(r-2)$-dimensional subspace $H_{\alpha} \subset \mathbb{P}^{2 r-1}$. We define $D_{u n b}^{r}$ as the closure of the locus of maps $\left[\mathbb{P}^{1}, \alpha\right] \in \bar{M}_{0,0}(L G(r, 2 r), 2)$ such that $H_{\alpha}$ intersects a fixed $(r+1)$-dimensional subspace of $\mathbb{P}^{2 r-1}$.

The main results in Lemma 6.6. Proposition 6.11, Theorem 6.14, Remark 6.13 and Corollary 6.17 can be summarized in the following statement:

Theorem 1.4. Let $\bar{M}_{0,0}(L G(r, 2 r), 2)$ be the Kontsevich space of conics in the Lagrangian Grassmannian $L G(r, 2 r)$, parametrizing Lagrangian subspaces of a $2 r$-dimensional symplectic vector space, with $r \geqslant 2$.

The effective cone $\operatorname{Eff}\left(\bar{M}_{0,0}(L G(r, 2 r), 2)\right)$ is generated by $\Delta^{r}$ and $D_{u n b}^{r}$, and the nef cone $\operatorname{Nef}\left(\bar{M}_{0,0}(L G(r, 2 r), 2)\right)$ is generated by $H_{\sigma_{2}}^{r}$ and $T^{r}$.

The Mori chamber decomposition of $\operatorname{Eff}\left(\bar{M}_{0,0}(L G(r, 2 r), 2)\right)$ has three chambers as displayed in the following picture:

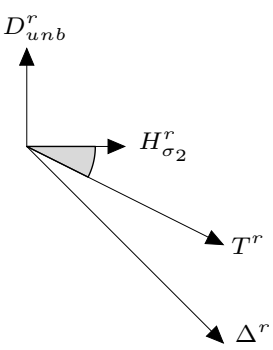

where $H_{\sigma_{2}}^{r} \sim \frac{1}{2}\left(\Delta^{r}+2 D_{u n b}^{r}\right)$ and $T^{r} \sim \Delta^{r}+D_{u n b}^{r}$. Furthermore, if $r \geqslant 2$ then $\operatorname{Mov}\left(\bar{M}_{0,0}(L G(r, 2 r), 2)\right)$ is generated by $T^{r}$ and $D_{u n b}^{r}$ while $\operatorname{Mov}\left(\bar{M}_{0,0}(L G(2,4), 2)\right)$ is generated by $T^{r}$ and $H_{\sigma_{2}}^{r}$.

The divisor $H_{\sigma_{2}}^{r}$ induces a birational morphism

$$
f_{H_{\sigma_{2}}^{r}}: \bar{M}_{0,0}(L G(r, 2 r), 2) \rightarrow \widetilde{\operatorname{Chow}}(L G(r, 2 r), 2)
$$

which is an isomorphism away form the locus $Q^{r}(1)$ of double covers of a line in $L G(r, 2 r)$, and contracts $Q^{r}(1)$ so that the locus of double covers with the same image maps to a point, where $\widetilde{C h o w}(L G(r, 2 r), 2)$ is the normalization of the Chow variety of conics in $L G(r, 2 r)$.

The divisor $T^{r}$ induces a morphism

$$
f_{T^{r}}: \bar{M}_{0,0}(L G(r, 2 r), 2) \rightarrow \bar{M}_{0,0}(L G(r, 2 r), 2,1)
$$

which is an isomorphism away from $\Delta^{r}$ and contracts the locus of maps with reducible domain $\left[C_{1} \cup C_{2}, \alpha\right]$ to $\alpha\left(C_{1} \cap C_{2}\right)$, where $\bar{M}_{0,0}(L G(r, 2 r), 2,1)$ is the moduli space of weighted stable maps to $L G(r, 2 r)$.

The birational model $X_{r}$ corresponding to the chamber delimited by $H_{\sigma_{2}}^{r}$ and $D_{u n b}^{r}$ is a fibration $X_{r} \rightarrow S G(r-$ $2,2 r)$ with fibers isomorphic to the Grassmannian $\mathbb{G}(2,4)$ parametrizing plane in $\mathbb{P}^{4}$, where $S G(r-2,2 r)$ is 
the symplectic Grassmannian parametrizing isotropic subspaces of dimension $r-2$. Moreover, D unb contracts $\bar{M}_{0,0}(L G(r, 2 r), 2)$ onto $S G(r-2,2 r)$.

Finally, $\bar{M}_{0,0}(L G(r, 2 r), 2)$ is Fano for $2 \leqslant r \leqslant 6$, weak Fano, that is $-K_{\bar{M}_{0,0}(L G(r, 2 r), 2)}$ is nef and big, for $r=7$, and $-K_{\bar{M}_{0,0}(L G(r, 2 r), 2)}$ is not ample for $r \geqslant 8$.

Moreover, Proposition 6.8, Remarks 1.2, 6.13 and Corollary 6.18 provide additional information for the case $r=2$.

Theorem 1.5. The following $S p(4)$-action

$$
\begin{aligned}
& S p(4) \times \bar{M}_{0,0}(L G(2,4), 2) \quad \longrightarrow \quad \bar{M}_{0,0}(L G(2,4), 2) \\
& (M,[C, \alpha]) \quad \longmapsto \quad\left[C, \wedge^{2} M \circ \alpha\right]
\end{aligned}
$$

induces on $\bar{M}_{0,0}(L G(2,4), 2)$ a structure of spherical variety. Furthermore, there exists an isomorphism

$$
\varphi: \bar{M}_{0,0}(L G(2,4), 2) \rightarrow \mathcal{S}_{4}
$$

where $\mathcal{S}_{4}$ is the wonderful compactification of the space of symplectic quadrics of $\mathbb{P}^{3}$, mapping a smooth conic $C \subset L G(2,4)$ to the quadric $\bigcup_{[L] \in C} L \subset \mathbb{P}^{3}$. The Cox ring $\operatorname{Cox}\left(\bar{M}_{0,0}(L G(2,4), 2)\right)$ is generated by the sections of $\Delta^{2}, D_{u n b}^{2}, H_{\sigma_{2}}^{2}, T^{2}$.

The moduli space $\bar{M}_{0,0}(L G(2,4), 2)$ identifies with the blow-up of $\mathbb{G}(1,4)$ along the Veronese $\mathcal{V}_{2}^{3}$. With this identification the morphism associated to $\mathrm{H}_{\sigma_{2}}^{2}$ is the blow-down and $\widehat{\operatorname{Chow}}(\operatorname{LG}(2,4), 2) \cong \mathbb{G}(1,4)$, while the morphism associated to $T^{2}$ is induced by the strict transform on $\mathcal{S}_{4}$ of the linear system of quadrics containing $\mathcal{V}_{2}^{3}$, and its image is a 6 -fold of degree 40 in $\mathbb{P}^{14}$ isomorphic to $\bar{M}_{0,0}(L G(2,4), 2,1)$.

Finally, PsAut $\left(\bar{M}_{0,0}(L G(2,4), 2)\right) \cong \operatorname{Aut}\left(\bar{M}_{0,0}(L G(2,4), 2)\right) \cong P S p(4)$ where PSp $(4)$ is the projective symplectic group, and $\operatorname{PsAut}\left(\bar{M}_{0,0}(L G(2,4), 2)\right)$ is the group of birational self-maps of $\bar{M}_{0,0}(L G(2,4), 2)$ inducing automorphisms in codimension one.

Organization of the paper. Throughout the paper we will work over an algebraically closed field $K$ of characteristic zero. In Section 2] as a warm-up we prove some of the main results in [Vai82], Vai84], using the techniques based on tangent cones computations that we will then apply to the more involved case of symplectic quadrics. In Section 3 we construct the wonderful compactification $\mathcal{S}_{2 r}$ of the space of symmetric and symplectic $2 r \times 2 r$ matrices. In Section 4 we study the Picard rank, the effective and the nef cones of $\mathcal{S}_{2 r}$. In Section 5 we compute the Mori chamber decomposition of the effective cone of $\mathcal{S}_{4}$ and $\mathcal{S}_{6}$. Finally, in Section 6 , taking advantage of the theory of complete symplectic quadrics, we investigate the birational geometry of Kontsevich spaces of conics in Lagrangian Grassmannians.

Acknowledgments. We thank very much Alex Casarotti, Massimiliano Mella, Giorgio Ottaviani and Jason Starr for useful discussions, and the referee for many helpful comments that helped us to improve the exposition and correct a mistake about the sphericity of $\bar{M}_{0,0}(L G(r, 2 r), 2)$ for $r>2$ in a first version of the paper.

The second named author is a member of the Gruppo Nazionale per le Strutture Algebriche, Geometriche e le loro Applicazioni of the Istituto Nazionale di Alta Matematica "F. Severi" (GNSAGA-INDAM).

\section{Complete quAdrics}

Let $V$ be a $K$-vector space of dimension $n+1$, and let $\mathbb{P}^{N}$ with $N=\left(\begin{array}{c}n+2 \\ 2\end{array}\right)-1$ be the projective space parametrizing quadratic forms on $\mathbb{P}^{n}=\mathbb{P}(V)$ up to a scalar multiple.

The line bundle $\mathcal{O}_{\mathbb{P}^{n}}(2)$ induces an embedding

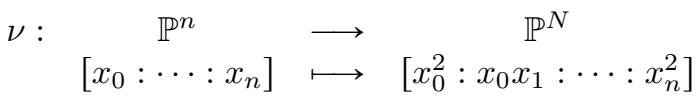

The image $\mathcal{V}_{2}^{n}=\nu\left(\mathbb{P}^{n}\right) \subset \mathbb{P}^{N}$ is the Veronese variety of dimension $n$ and degree $2^{n}$. We will denote by $\left[z_{0,0}: \cdots:\right.$ $\left.z_{n, n}\right]$ the homogeneous coordinates on $\mathbb{P}^{N}$, where $z_{i, j}$ corresponds to the product $x_{i} x_{j}$.

Secant varieties. Given an irreducible and reduced non-degenerate variety $X \subset \mathbb{P}^{N}$, and a positive integer $h \leqslant N$ we denote by $\operatorname{Sec}_{h}(X)$ the $h$-secant variety of $X$. This is the subvariety of $\mathbb{P}^{N}$ obtained as the closure of the union of all $(h-1)$-planes $\left\langle x_{1}, \ldots, x_{h}\right\rangle$ spanned by $h$ general points of $X$.

A point $p \in \mathbb{P}^{N}$ can be represented by an $(n+1) \times(n+1)$ symmetric matrix $Z$. The Veronese variety $\mathcal{V}_{2}^{n}$ is the locus of rank one matrices. More generally, $p \in \operatorname{Sec}_{h}\left(\mathcal{V}_{2}^{n}\right)$ if and only if $Z$ can be written as a linear combination 
of $h$ rank one matrices that is if and only if $\operatorname{rank}(Z) \leqslant h$. If $p=\left[z_{0,0}: \cdots: z_{n, n}\right]$ then we may write

$$
Z=\left(\begin{array}{ccc}
z_{0,0} & \ldots & z_{0, n} \\
\vdots & \ddots & \vdots \\
z_{0, n} & \ldots & z_{n, n}
\end{array}\right)
$$

Then, the ideal of $\operatorname{Sec}_{h}\left(\mathcal{V}_{2}^{n}\right)$ is generated by the $(h+1) \times(h+1)$ minors of $Z$.

By [Mas20a, Lemma 3.3] the $S L(n+1)$-action

$$
\begin{array}{ccc}
S L(n+1) \times \mathbb{P}^{n} & \longrightarrow & \mathbb{P}^{n} \\
(M,[v]) & \longmapsto & {[M v]}
\end{array}
$$

induces the $S L(n+1)$-action on $\mathbb{P}^{N}$ given by

$$
\begin{array}{clc}
S L(n+1) \times \mathbb{P}^{N} & \longrightarrow & \mathbb{P}^{N} \\
(M, Z) & \longmapsto & M Z M^{t}
\end{array}
$$

The orbit closures of the action (2.2) are precisely the secant varieties $\operatorname{Sec}_{h}\left(\mathcal{V}_{2}^{n}\right)$. Now, let us recall the notion of spherical and wonderful variety.

Definition 2.3. A spherical variety is a normal variety $X$ together with an action of a connected reductive affine algebraic group $\mathscr{G}$, a Borel subgroup $\mathscr{B} \subset \mathscr{G}$, and a base point $x_{0} \in X$ such that the $\mathscr{B}$-orbit of $x_{0}$ in $X$ is a dense open subset of $X$.

Let $\left(X, \mathscr{G}, \mathscr{B}, x_{0}\right)$ be a spherical variety. We distinguish two types of $\mathscr{B}$-invariant prime divisors: a boundary divisor of $X$ is a $\mathscr{G}$-invariant prime divisor on $X$, a color of $X$ is a $\mathscr{B}$-invariant prime divisor that is not $\mathscr{G}$-invariant. We will denote by $\mathcal{B}(X)$ and $\mathcal{C}(X)$ respectively the set of boundary divisors and colors of $X$.

For instance, any toric variety is a spherical variety with $\mathscr{B}=\mathscr{G}$ equal to the torus. For a toric variety there are no colors, and the boundary divisors are the usual toric invariant divisors.

Definition 2.4. A wonderful variety is a smooth projective variety $X$ with the action of a semi-simple simply connected group $\mathscr{G}$ such that:

- there is a point $x_{0} \in X$ with open $\mathscr{G}$ orbit and such that the complement $X \backslash \mathscr{G} \cdot x_{0}$ is a union of prime divisors

$E_{1}, \cdots, E_{r}$ having simple normal crossing;

- the closures of the $\mathscr{G}$-orbits in $X$ are the intersections $\bigcap_{i \in I} E_{i}$ where $I$ is a subset of $\{1, \ldots, r\}$.

As proven by D. Luna in Lun96 wonderful varieties are in particular spherical. Note that $\mathbb{P}^{N}$ is not a wonderful compactification of $S L(n+1) / H$, where $H$ is the stabilizer of the identity matrix with respect to the $S L(n+1)$ action in (2.2), since for instance the orbit closure $\operatorname{Sec}_{n}\left(\mathcal{V}_{2}^{n}\right)$ is a non smooth divisor. In order to get a wonderful compactification we must consider the space of complete quadrics that we now describe. The space of complete quadrics is the closure of the graph of the rational map

$$
\begin{array}{ccc}
\mathbb{P}\left(\operatorname{Sym}^{2} V\right) & -\rightarrow & \mathbb{P}\left(\operatorname{Sym}^{2} \wedge^{2} V\right) \times \cdots \times \mathbb{P}\left(\operatorname{Sym}^{2} \wedge^{n} V\right) \\
Z & \longmapsto & \left(\wedge^{2} Z, \ldots, \wedge^{n} Z\right)
\end{array}
$$

By [Vai82, Theorem 6.3] the space of complete quadrics can be constructed as a sequence of blow-ups as follows.

Construction 2.5. Let us consider the following sequence of blow-ups:

- $\mathcal{Q}(n)_{1}$ is the blow-up of $\mathcal{Q}(n)_{0}:=\mathbb{P}^{N}$ along the Veronese variety $\mathcal{V}_{2}^{n}$;

- $\mathcal{Q}(n)_{2}$ is the blow-up of $\mathcal{Q}(n)_{1}$ along the strict transform of $\operatorname{Sec}_{2}\left(\mathcal{V}_{2}^{n}\right)$;

- $\mathcal{Q}(n)_{i}$ is the blow-up of $\mathcal{Q}(n)_{i-1}$ along the strict transform of $\operatorname{Sec}_{i}\left(\mathcal{V}_{2}^{n}\right)$;

- $\mathcal{Q}(n)_{n-1}$ is the blow-up of $\mathcal{Q}(n)_{n-2}$ along the strict transform of $\operatorname{Sec}_{n-1}\left(\mathcal{V}_{2}^{n}\right)$.

Let $f_{i}: \mathcal{Q}(n)_{i} \rightarrow \mathcal{Q}(n)_{i-1}$ be the blow-up morphism. We will denote by $E_{i}^{q}$ both the exceptional divisor of $f_{i}$ and its strict transforms in the subsequent blow-ups. We will denote by $\mathcal{Q}(n)$ the last blow-up $\mathcal{Q}(n)_{n-1}$ and by $f: \mathcal{Q}(n) \rightarrow \mathbb{P}^{N}$ the composition of the $f_{i}$.

Then for any $i=1, \ldots, n-1$ the variety $\mathcal{Q}(n)_{i}$ is smooth, the strict transform of $\operatorname{Sec}_{i+1}\left(\mathcal{V}_{2}^{n}\right)$ in $\mathcal{Q}(n)_{i}$ is smooth, and the divisor $E_{1}^{q} \cup E_{2}^{q} \cup \cdots \cup E_{i}^{q}$ in $\mathcal{Q}(n)_{i}$ is simple normal crossing. Furthermore, the variety $\mathcal{Q}(n)$ is isomorphic to the space of complete $(n-1)$-dimensional quadrics.

In particular, $\mathcal{Q}(n)$ is a wonderful compactification of the homogeneous space $S L(n+1) / S O(n+1)$. We now recall some facts about the varieties $\operatorname{Sec}_{h}\left(\mathcal{V}_{2}^{n}\right)$. 
Remark 2.6. Recall that $\operatorname{Sec}_{h}\left(\mathcal{V}_{2}^{n}\right)$ identifies with the variety parametrizing $(n+1) \times(n+1)$ symmetric matrices modulo scalar of rank at most $h$. An argument similar to the one used to estimate the dimension of the spaces of matrices, not necessarily symmetric, of rank at most $h$ in [Har95, Example 12.1] shows that

$$
\operatorname{dim}\left(\operatorname{Sec}_{h}\left(\mathcal{V}_{2}^{n}\right)\right)=\frac{2 n h-h^{2}+3 h-2}{2}
$$

for $h \leqslant n$. Furthermore, identifying $\operatorname{Sec}_{h}\left(\mathcal{V}_{2}^{n}\right)$ with the variety parametrizing $(n+1) \times(n+1)$ symmetric matrices modulo scalar of corank at least $n+1-h$, by [HT84, Proposition 12(b)] we get that the degree of $\operatorname{Sec}_{h}\left(\mathcal{V}_{2}^{n}\right)$ is given by

$$
\operatorname{deg}\left(\operatorname{Sec}_{h}\left(\mathcal{V}_{2}^{n}\right)\right)=\prod_{i=0}^{n-h} \frac{\left(\begin{array}{c}
n+1+i \\
n+1-h-i
\end{array}\right)}{\left(\begin{array}{c}
2 i+1 \\
i
\end{array}\right)}
$$

In particular, for $h=n$ we get $n+1$, and for $h=1$ we get $2^{n}$.

Proposition 2.7. The tangent cone of $\operatorname{Sec}_{h}\left(\mathcal{V}_{2}^{n}\right)$ at a point $p \in \operatorname{Sec}_{k}\left(\mathcal{V}_{2}^{n}\right) \backslash \operatorname{Sec}_{k-1}\left(\mathcal{V}_{2}^{n}\right)$ for $k \leqslant h$ is a cone with

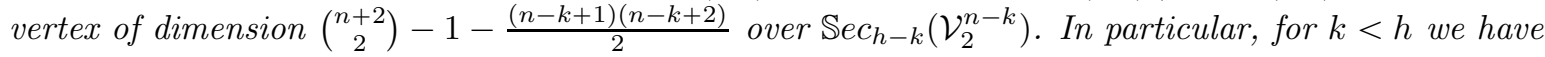

$$
\operatorname{mult}_{\operatorname{Sec}_{k}\left(\mathcal{V}_{2}^{n}\right) \backslash \operatorname{Sec}_{k-1}\left(\mathcal{V}_{2}^{n}\right)} \operatorname{Sec}_{h}\left(\mathcal{V}_{2}^{n}\right)=\prod_{i=0}^{n-h} \frac{\left(\begin{array}{c}
n-k+1+i \\
n+1-h-i
\end{array}\right)}{\left(\begin{array}{c}
2 i+1 \\
i
\end{array}\right)}
$$

and $\operatorname{Sing}\left(\operatorname{Sec}_{h}\left(\mathcal{V}_{2}^{n}\right)\right)=\operatorname{Sec}_{h-1}\left(\mathcal{V}_{2}^{n}\right)$.

Proof. We compute the tangent cone of $\operatorname{Sec}_{h}\left(\mathcal{V}_{2}^{n}\right)$ at

$$
p_{k}=\left(\begin{array}{cc}
I_{k, k} & 0_{k, n+1-k} \\
0_{n+1-k, k} & 0_{n+1-k, n+1-k}
\end{array}\right)
$$

where $I_{k, k}$ is the $k \times k$ identity matrix. Consider the affine chart $z_{0,0} \neq 0$ and the change of coordinates $z_{i, i} \mapsto z_{i, i}-1$ for $i=1, \ldots, k-1, z_{i, j} \mapsto z_{i, j}$ if $i \neq j$. Then the matrix $Z$ in (2.1) takes the following form

$$
\left(\begin{array}{ccccccc}
1 & z_{0,1} & \ldots & z_{0, k-1} & z_{0, k} & \ldots & z_{0, n} \\
z_{0,1} & z_{1,1}-1 & \ldots & z_{1, k-1} & z_{1, k} & \ldots & z_{1, n} \\
\vdots & \vdots & \ddots & \vdots & \vdots & \ddots & \vdots \\
z_{0, k-1} & z_{1, k-1} & \ldots & z_{k-1, k-1}-1 & z_{k-1, k} & \ldots & z_{k-1, n} \\
z_{0, k} & z_{1, k} & \ldots & z_{k-1, k} & z_{k, k} & \ldots & z_{k, n} \\
\vdots & \vdots & \ddots & \vdots & \vdots & \ddots & \vdots \\
z_{0, n} & z_{1, n} & \ldots & z_{k-1, n} & z_{k, n} & \ldots & z_{n, n}
\end{array}\right)
$$

Recall that $\operatorname{Sec}_{h}\left(\mathcal{V}_{2}^{n}\right) \subseteq \mathbb{P}^{N}$ is cut out by the $(h+1) \times(h+1)$ minors of $Z$. Now, the lowest degree terms of these minors are given by the $(h+1-k) \times(h+1-k)$ minors of the following matrix

$$
\left(\begin{array}{ccc}
z_{k, k} & \ldots & z_{k, n} \\
\vdots & \ddots & \vdots \\
z_{k, n} & \cdots & z_{n, n}
\end{array}\right)
$$

Therefore, the tangent cone $T C_{p_{k}} \operatorname{Sec}_{h}\left(\mathcal{V}_{2}^{n}\right)$ is contained in the cone $C$ over $\operatorname{Sec}_{h-k}\left(\mathcal{V}_{2}^{n-k}\right)$ with vertex the linear subspace of $\mathbb{P}^{N}$ given by $\left\{z_{k, k}=\cdots=z_{k, n}=z_{k+1, k+1}=\cdots=z_{k+1, n}=\cdots=z_{n, n}=0\right\}$. Now, Remark 2.6 yields

$$
\operatorname{dim}(C)=\left(\begin{array}{c}
n+2 \\
2
\end{array}\right)-1-\frac{(n-k+1)(n-k+2)}{2}+\operatorname{dim}\left(\operatorname{Sec}_{h-k}\left(\mathcal{V}_{2}^{n-k}\right)\right)+1=\operatorname{dim}\left(\operatorname{Sec}_{h}\left(\mathcal{V}_{2}^{n}\right)\right)
$$

and hence $T C_{p_{k}} \operatorname{Sec}_{h}\left(\mathcal{V}_{2}^{n}\right)=C$. Finally, to get the formula for the multiplicity it is enough to observe that

$$
\operatorname{mult}_{p_{k}} \operatorname{Sec}_{h}\left(\mathcal{V}_{2}^{n}\right)=\operatorname{mult}_{p_{k}} T C_{p_{k}} \operatorname{Sec}_{h}\left(\mathcal{V}_{2}^{n}\right)=\operatorname{deg}\left(\operatorname{Sec}_{h-k}\left(\mathcal{V}_{2}^{n-k}\right)\right)
$$

and to apply the formula for the degree of the secant varieties of $\mathcal{V}_{2}^{n}$ in Remark 2.6.

We will need the following result on fibrations with smooth fibers on a smooth base.

Proposition 2.8. Let $f: X \rightarrow Y$ be a surjective morphism of varieties over an algebraically closed field with equidimensional smooth fibers. If $Y$ is smooth then $X$ is smooth as well. 
Proof. By [Sch10, Theorem 3.3.27] the morphism $f: X \rightarrow Y$ is flat. Finally, since all the fibers of $f: X \rightarrow Y$ are smooth and of the same dimension [Mum99, Theorem 3', Chapter III, Section 10] yields that $X$ is smooth.

However, a direct proof is at hand and we present it in what follows. Since the problem is local on both $X$ and $Y$ we may assume that $X \subset K^{N}$ is an affine variety cut out by polynomials $g_{1}, \ldots, g_{a}, Y=K^{m}$, and $f: X \rightarrow Y$ is given by $f(x)=\left(f_{1}(x), \ldots, f_{m}(x)\right)$.

Consider a point $p \in X$. Without loss of generality we may assume that $f(p)=0$. Then the fiber $X_{0}$ of $f$ through $p$ is given by

$$
f^{-1}(0)=\left\{x \in K^{N} \mid g_{1}(x)=\cdots=g_{a}(x)=f_{1}(x)=\cdots=f_{m}(x)=0\right\} .
$$

Now, since $X_{0}$ is smooth at $p$ there are $b \leqslant a$ polynomials among $g_{1}, \ldots, g_{a}$ and $l \leqslant m$ polynomials among $f_{1}, \ldots, f_{m}$ such that $b+l=m+N-\operatorname{dim}(X)$ and the vectors

$$
\left(\nabla g_{1}\right)(p), \ldots,\left(\nabla g_{b}\right)(p),\left(\nabla f_{1}\right)(p), \ldots,\left(\nabla f_{l}\right)(p)
$$

are linearly independent. Now, $l \leqslant m$ yields $b \geqslant N-\operatorname{dim}(X)$. On the other hand, $X$ is irreducible of codimension $N-\operatorname{dim}(X)$ and hence $b \leqslant N-\operatorname{dim}(X)$. We conclude that $b=N-\operatorname{dim}(X)$ and the vectors

$$
\left(\nabla g_{1}\right)(p), \ldots,\left(\nabla g_{N-\operatorname{dim}(X)}\right)(p)
$$

are linearly independent. So $X$ is smooth at $p$.

Notation 2.9. We will denote by $\operatorname{Sec}_{h}\left(\mathcal{V}_{2}^{n}\right)^{i}$ the strict transform of $\operatorname{Sec}_{h}\left(\mathcal{V}_{2}^{n}\right)$ in $\mathcal{Q}(n)_{i}$ for $h>i$. Furthermore, as already said in Construction 2.5. for simplicity of notation we will denote by $E_{i}^{q}$ both the exceptional divisor of $f_{i}$ and its strict transforms in the subsequent blow-ups.

In the following we will analyze the geometry of the $S L(n+1)$-orbits in the blow-ups $\mathcal{Q}(n)_{i}$ in Construction 2.5 ,

Proposition 2.10. For any $i=0, \ldots, n-1$ the variety $\mathcal{Q}(n)_{i}$ is smooth and the divisors $E_{1}^{q}, \ldots, E_{i}^{q}$ are smooth and intersect transversally in $\mathcal{Q}(n)_{i}$. Furthermore, the strict transform $\mathbb{S e c}_{i+1}\left(\mathcal{V}_{2}^{n}\right)^{i}$ of $\operatorname{Sec}_{i+1}\left(\mathcal{V}_{2}^{n}\right)$ in $\mathcal{Q}(n)_{i}$ is smooth and the intersections among $\operatorname{Sec}_{i+1}\left(\mathcal{V}_{2}^{n}\right)^{i}, E_{1}^{q}, \ldots, E_{i}^{q}$ are transversal. The closures of the orbits of the $S L(n+1)$ action on $\mathcal{Q}(n)_{i}$ induced by (2.2) are given by all the possible intersections of $E_{1}^{q}, \ldots, E_{i}^{q}, \operatorname{Sec}_{i+1}\left(\mathcal{V}_{2}^{n}\right)^{i}, \ldots, \operatorname{Sec}_{n}\left(\mathcal{V}_{2}^{n}\right)^{i}$ and $\mathcal{Q}(n)_{i}$ itself.

In particular, the variety $\mathcal{Q}(n)$ is smooth, the divisors $E_{1}^{q}, \ldots, E_{n-1}^{q}, \operatorname{Sec}_{n}\left(\mathcal{V}_{2}^{n}\right)^{n-1}$ are smooth and the intersections among them are transversal, the closures of the orbits of the $S L(n+1)$-action on $\mathcal{Q}(n)$ induced by (2.2) are given by all the possible intersections of the divisors $E_{1}^{q}, \ldots, E_{n-1}^{q}, \mathbb{S e c}_{n}\left(\mathcal{V}_{2}^{n}\right)^{n-1}$ and $\mathcal{Q}(n)$ itself. Hence, $\mathcal{Q}(n)$ is wonderful.

Proof. We will proceed as follows. For $i=0,1$ we will prove the statement for any $n$. Then we will prove that if for $i<j$ the statement holds for any $n$ then it also holds for $i=j$ and any $n$. This will prove the statement for any $n \geqslant 1$ and $i=0, \ldots, n-1$.

For $i=0$ we have $\mathcal{Q}(n)_{0} \cong \mathbb{P}^{N}$, there are no exceptional divisors, and the closures of the orbits of the action (2.2) are the secant varieties of $\mathcal{V}_{2}^{n}$. Therefore, for $i=0$ the statements holds for any $n$. Even though we could use the case $i=0$ as the first step of the proof, to get acquainted with the arguments we will apply, we develop in full detail the case $i=1$ as well.

The variety $\mathcal{Q}(n)_{1}$ is the blow-up of $\mathbb{P}^{N}$ along the Veronese variety $\mathcal{V}_{2}^{n}$. Hence it is smooth. By Proposition 2.7 $\operatorname{Sec}_{2}\left(\mathcal{V}_{2}^{n}\right)$ is smooth away from $\mathcal{V}_{2}^{n}$ and $\operatorname{Sec}_{2}\left(\mathcal{V}_{2}^{n}\right)^{1} \cap E_{1}^{q} \rightarrow \mathcal{V}_{2}^{n}$ is a fibration whose fibers are isomorphic to $\mathcal{V}_{2}^{n-1}$. Hence, Proposition 2.8 yields that $\operatorname{Sec}_{2}\left(\mathcal{V}_{2}^{n}\right)^{1} \cap E_{1}^{q}$ is smooth and since $\operatorname{dim}\left(\operatorname{Sec}_{2}\left(\mathcal{V}_{2}^{n}\right)^{1} \cap E_{1}^{q}\right)=n+n-1=2 n-1=$ $\operatorname{dim}\left(\operatorname{Sec}_{2}\left(\mathcal{V}_{2}^{n}\right)^{1}\right)-1$ we conclude that $\operatorname{Sec}_{2}\left(\mathcal{V}_{2}^{n}\right)^{1}$ is smooth and the intersection $\mathbb{S e c}_{2}\left(\mathcal{V}_{2}^{n}\right)^{1} \cap E_{1}^{q}$ is transversal.

Now, via the action of $S L(n+1)$ in (2.2) we can translate any fiber of $E_{1}^{q}$ over $\mathcal{V}_{2}^{n}$ to any other fiber. Fix one such fiber $E_{1, p}^{q}$. By Proposition 2.7 we have that $\operatorname{Sec}_{h}\left(\mathcal{V}_{2}^{n}\right)^{1} \cap E_{1, p}^{q}=\operatorname{Sec}_{h-1}\left(\mathcal{V}_{2}^{n-1}\right)$ and the action of $S L(n+1)$ in (2.2) restricts on $E_{1, p}^{q}$ to the corresponding action of $S L(n)$. This proves the statement about the orbits for $\mathcal{Q}(n)_{1}$ for any $n \geqslant 1$.

Assume that for any $i<j$ the statement holds for any $n$. Since $\mathcal{Q}(n)_{j-1}$ and $\operatorname{Sec}_{j}\left(\mathcal{V}_{2}^{n}\right)^{j-1} \subset \mathcal{Q}(n)_{j-1}$ are smooth the blow-up $\mathcal{Q}(n)_{j}$ of $\mathcal{Q}(n)_{j-1}$ along $\operatorname{Sec}_{j}\left(\mathcal{V}_{2}^{n}\right)^{j-1}$ is smooth as well. Furthermore, since all the intersections among $\operatorname{Sec}_{j}\left(\mathcal{V}_{2}^{n}\right)^{j-1}, E_{1}^{q}, \ldots, E_{j-1}^{q}$ in $\mathcal{Q}(n)_{j-1}$ are transversal we have that all the intersections among $E_{1}^{q}, \ldots, E_{j}^{q}$ in $\mathcal{Q}(n)_{j}$ are transversal as well.

Now, consider an intersection of the following form $\operatorname{Sec}_{j+1}\left(\mathcal{V}_{2}^{n}\right)^{j} \cap E_{j_{1}}^{q} \cap \cdots \cap E_{j_{t}}^{q}$. By Proposition 2.7 the restriction of the blow-down morphism

$$
\operatorname{Sec}_{j+1}\left(\mathcal{V}_{2}^{n}\right)^{j} \cap E_{j_{1}}^{q} \cap \cdots \cap E_{j_{t}}^{q} \rightarrow E_{j_{1}}^{q} \cap \cdots \cap E_{j_{t-1}}^{q} \cap \operatorname{Sec}_{j_{t}}\left(\mathcal{V}_{2}^{n}\right)^{j_{t}-1}
$$


has fibers isomorphic to $\operatorname{Sec}_{j-j_{t}+1}\left(\mathcal{V}^{n-j_{t}}\right)^{j-j_{t}}$. Since both $E_{j_{1}}^{q} \cap \cdots \cap E_{j_{t-1}}^{q} \cap \mathbb{S e c}_{j_{t}}\left(\mathcal{V}_{2}^{n}\right)^{j_{t}-1}$ and $\mathbb{S e c}_{j-j_{t}-1}\left(\mathcal{V}^{n-j_{t}}\right)^{j-j_{t}}$ are smooth Proposition 2.8 yields that $\operatorname{Sec}_{j+1}\left(\mathcal{V}_{2}^{n}\right)^{j} \cap E_{j_{1}}^{q} \cap \cdots \cap E_{j_{t}}^{q}$ is smooth as well. Moreover, note that

$$
\operatorname{dim}\left(\operatorname{Sec}_{j+1}\left(\mathcal{V}_{2}^{n}\right)^{j} \cap E_{j_{1}}^{q} \cap \cdots \cap E_{j_{t}}^{q}\right)=\operatorname{dim}\left(E_{j_{1}}^{q} \cap \cdots \cap E_{j_{t-1}}^{q} \cap \operatorname{Sec}_{j_{t}}\left(\mathcal{V}_{2}^{n}\right)^{j_{t}-1}\right)+\operatorname{dim}\left(\mathbb{S e c}_{j-j_{t}+1}\left(\mathcal{V}^{n-j_{t}}\right)^{j-j_{t}}\right)
$$

and

$$
\operatorname{dim}\left(E_{j_{1}}^{q} \cap \cdots \cap E_{j_{t-1}}^{q} \cap \operatorname{Sec}_{j_{t}}\left(\mathcal{V}_{2}^{n}\right)^{j_{t}-1}\right)=\frac{2 n j_{t}-j_{t}^{2}+3 j_{t}-2}{2}-(t-1)
$$

yield that $\operatorname{dim}\left(\operatorname{Sec}_{j+1}\left(\mathcal{V}_{2}^{n}\right)^{j} \cap E_{j_{1}}^{q} \cap \cdots \cap E_{j_{t}}^{q}\right)$ is given by

$$
\begin{aligned}
& \frac{2 n j_{t}-j_{t}^{2}+3 j_{t}-2}{2}-(t-1)+\frac{2\left(n-j_{t}\right)\left(j-j_{t}+1\right)-\left(j-j_{t}+1\right)^{2}+3\left(j-j_{t}+1\right)-2}{2}= \\
& \frac{2 n(j+1)-(j+1)^{2}+3(j+1)-2}{2}-t=\operatorname{dim}\left(\operatorname{Sec}_{j+1}\left(\mathcal{V}_{2}^{n}\right)^{j}\right)-t
\end{aligned}
$$

and hence the intersection $\operatorname{Sec}_{j+1}\left(\mathcal{V}_{2}^{n}\right)^{j} \cap E_{j_{1}}^{q} \cap \cdots \cap E_{j_{t}}^{q}$ is transversal.

In the following we prove the claim about the orbit closures. If an orbit closure in $\mathcal{Q}(n)_{i}$ is not contained in the exceptional divisor $E_{i}^{q}$ then it is the strict transform of an orbit closure in $\mathcal{Q}(n)_{i}$, and hence it is given as an intersection among $E_{1}^{q}, \ldots, E_{i-1}^{q}, \operatorname{Sec}_{i+1}\left(\mathcal{V}_{2}^{n}\right)^{i}, \ldots, \operatorname{Sec}_{n}\left(\mathcal{V}_{2}^{n}\right)^{i}$.

Now, let us analyze the orbit closures in the exceptional divisor $E_{i}^{q}$. The fibers of $E_{i}^{q}$ over $\mathbb{S e c}_{i}\left(\mathcal{V}_{2}^{n}\right)^{i-1}$ are projective spaces of dimension

$$
N_{n-i}=\left(\begin{array}{c}
n-i+2 \\
2
\end{array}\right)-1 .
$$

Moreover, $S L(n+1)$ acts transitively on fibers that lie over the same orbit in $\mathcal{Q}(n)_{i-1}$. Note that by Lemma 2.7 $\operatorname{Sec}_{h}\left(\mathcal{V}_{2}^{n}\right)^{i}$ intersects each of these $N_{n-i}$-dimensional projective spaces along $\operatorname{Sec} c_{h-i}\left(\mathcal{V}_{2}^{n-i}\right)$, and the $S L(n+1)$-action on $\mathcal{Q}(n)_{i}$ in (2.2) induces the corresponding $S L(n-i+1)$-action on the fibers of $E_{i}^{q}$. Finally, the statement on the orbit closures in $\mathcal{Q}(n)_{i-1}$ follows then from the statement on the orbit closures in $\mathcal{Q}(n-i)_{0}$.

\section{Complete Symmetric SymplectiC FORMS}

From now on we will consider the case $n+1=2 r$ even. Let $S p(2 r)$ be the symplectic group of $2 r \times 2 r$ symplectic matrices, that is

$$
S p(2 r)=\left\{M \in \operatorname{Hom}(V, V) \mid M^{t} \Omega M=\Omega\right\}
$$

where

$$
\Omega=\left(\begin{array}{cc}
0 & I_{r, r} \\
-I_{r, r} & 0
\end{array}\right)
$$

is the standard symplectic form. Over an algebraically closed field of characteristic zero the symplectic group is a non-compact, irreducible, simply connected, simple Lie group.

Remark 3.2. Let us write a $2 r \times 2 r$ matrix $M$ as

$$
M=\left(\begin{array}{ll}
A & B \\
C & D
\end{array}\right)
$$

where $A, B, C, D$ are four $r \times r$ matrices. The condition of being symplectic translates then into the following system of equations

Considering the transformation

$$
\left\{\begin{array}{l}
-C^{t} A+A^{t} C=0_{r, r} ; \\
-C^{t} B+A^{t} D=I_{r, r} ; \\
-D^{t} A+B^{t} C=-I_{r, r} \\
-D^{t} B+B^{t} D=0_{r, r}
\end{array}\right.
$$

$$
\left(\begin{array}{cc}
A & B \\
C & D
\end{array}\right) \mapsto\left(\begin{array}{cc}
A-I_{r, r} & B \\
C & D-I_{r, r}
\end{array}\right)
$$

we get the following relations for the tangent space of $S p(2 r)$ at the identity

$$
A=-D^{t}, B=B^{t}, C=C^{t} .
$$

Hence, the tangent space of $S p(2 r)$ at the identity is the Lie algebra $\mathfrak{s p}(2 r, K)$ consisting of $2 r \times 2 r$ matrices of the form

$$
\left(\begin{array}{cc}
A & B \\
C & -A^{t}
\end{array}\right)
$$

with $C$ and $B$ symmetric. In particular, $\operatorname{dim}(S p(2 r))=r^{2}+2 \frac{r(r+1)}{2}=r(2 r+1)$. 
Remark 3.4. By Ou12, Section 1] the Borel subgroup of the symplectic group can be described as follows:

$$
\mathscr{B}=\left\{\left(\begin{array}{ll}
A & 0_{r, r} \\
B & A^{-t}
\end{array}\right) \text { with } A^{t} B=B^{t} A\right\}
$$

where $A \in G L(r)$ is lower triangular and $B$ is a general $r \times r$ matrix. Now, $S p(2 r)$ is a subgroup of $S L(n+1)$ and the $S L(n+1)$-action (2.2) restricts to the following $S p(2 r)$-action:

$$
\begin{array}{ccc}
S p(2 r) \times \mathbb{P}^{N} & \longrightarrow & \mathbb{P}^{N} \\
(M, Z) & \longmapsto & M Z M^{t}
\end{array}
$$

We denote by $O_{2 r}$ the $S p(2 r)$-orbit of the identity in $\mathbb{P}^{N}$ and by $X_{2 r}=\overline{O_{2 r}} \subseteq \mathbb{P}^{N}$ its closure.

Proposition 3.6. Let $Y_{k}=\overline{O_{k}} \subset \mathbb{P}^{N}$ be the closure of the $S p(2 r)$-orbit of the matrix

$$
I_{k}=\left(\begin{array}{cc}
I_{k, k} & 0_{k, 2 r-k} \\
0_{2 r-k, k} & 0_{2 r-k, 2 r-k}
\end{array}\right)
$$

via the action in (2.2). If $k \leqslant r$ then

$$
\operatorname{dim}\left(Y_{k}\right)=r(2 r+1)-\frac{k(k-1)}{2}-r(r-k)-\frac{r(r+1)}{2}-\frac{(r-k)(r-k+1)}{2}-1=2 r k+k-k^{2}-1 .
$$

Finally, $\operatorname{dim}\left(Y_{2 r}\right)=r(r+1)$.

Proof. Our aim is to compute the dimension of the stabilizer $H \subset S p(2 r)$ of $I_{k}$. Consider the incidence correspondence

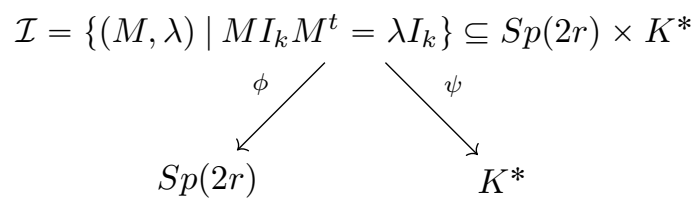

Note that the fibers of $\psi$ are isomorphic subgroups of $S p(2 r)$. We will compute the dimension of $H_{1}=\psi^{-1}(1)$ and then the dimension of $H=\phi(\mathcal{I})$ will be given by

$$
\operatorname{dim}(H)=\operatorname{dim}(\mathcal{I})=\operatorname{dim}\left(H_{1}\right)+1 .
$$

Consider first the case $k \leqslant r$. Subdivide as usual the matrices in $S p(2 r)$ in four $r \times r$ blocks and write the matrix whose orbit we want to study as

$$
\left(\begin{array}{cc}
Z_{k} & 0_{r, r} \\
0_{r, r} & 0_{r, r}
\end{array}\right)
$$

where $Z_{k}$ is the following $r \times r$ matrix

$$
Z_{k}=\left(\begin{array}{cc}
I_{k, k} & 0_{k, r-k} \\
0_{r-k, k} & 0_{r-k, r-k}
\end{array}\right)
$$

Now, we have

$$
\left(\begin{array}{cc}
A & B \\
C & D
\end{array}\right)\left(\begin{array}{cc}
Z_{k} & 0_{r, r} \\
0_{r, r} & 0_{r, r}
\end{array}\right)\left(\begin{array}{cc}
A^{t} & C^{t} \\
B^{t} & D^{t}
\end{array}\right)=\left(\begin{array}{cc}
A Z_{k} A^{t} & A Z_{k} C^{t} \\
C Z_{k} A^{t} & C Z_{k} C^{t}
\end{array}\right)
$$

Subdivide the matrix $A$ in blocks as follows

$$
A=\left(\begin{array}{cc}
A_{k, k} & A_{k, r-k} \\
A_{r-k, k} & A_{r-k, r-k}
\end{array}\right) .
$$

Then

$$
\left(\begin{array}{cc}
A_{k, k} & A_{k, r-k} \\
A_{r-k, k} & A_{r-k, r-k}
\end{array}\right)\left(\begin{array}{cc}
I_{k, k} & 0_{k, r-k} \\
0_{r-k, k} & 0_{r-k, r-k}
\end{array}\right)\left(\begin{array}{cc}
A_{k, k}^{t} & A_{r-k, k}^{t} \\
A_{k, r-k}^{t} & A_{r-k, r-k}^{t}
\end{array}\right)=\left(\begin{array}{cc}
A_{k, k} A_{k, k}^{t} & A_{k, k} A_{r-k, k}^{t} \\
A_{r-k, k} A_{k, k}^{t} & A_{r-k, k} A_{r-k, k}^{t}
\end{array}\right) .
$$

Therefore, considering the transformation (3.3) we get the following relations for the tangent space of $H_{1}$ at the identity

$$
A_{k, k}=-A_{k, k}^{t}, A_{r-k, k}=0_{r-k, k} .
$$

Moreover, subdividing $C$ as we did for $A$, we get that the matrix

$$
\left(\begin{array}{cc}
A_{k, k} & A_{k, r-k} \\
A_{r-k, k} & A_{r-k, r-k}
\end{array}\right)\left(\begin{array}{cc}
I_{k, k} & 0_{k, r-k} \\
0_{r-k, k} & 0_{r-k, r-k}
\end{array}\right)\left(\begin{array}{cc}
C_{k, k}^{t} & C_{r-k, k}^{t} \\
C_{k, r-k}^{t} & C_{r-k, r-k}^{t}
\end{array}\right)=\left(\begin{array}{cc}
A_{k, k} C_{k, k}^{t} & A_{k, k} C_{r-k, k}^{t} \\
A_{r-k, k} C_{k, k}^{t} & A_{r-k, k} C_{r-k, k}^{t}
\end{array}\right)
$$

must be zero. This yields the following further relations for the tangent space of $H_{1}$ at the identity

$$
C_{k, k}=0_{k, k}, C_{r-k, k}=0_{r-k, k} .
$$


Plugging-in the relations for the tangent space at the identity of $S p(2 r)$ in Remark 3.2 we get that the tangent space at the identity of $H_{1}$ is given by matrices of the form

$$
\left(\begin{array}{cc}
A & B \\
C & -A^{t}
\end{array}\right)
$$

where $B=B^{t}$ and $C=C^{t}$. Note that $C=C^{t}, C_{k, k}=0_{k, k}, C_{r-k, k}=0_{r-k, k}$ yield $C_{k, r-k}=0_{k, r-k}$ and $C_{r-k, r-k}=C_{r-k, r-k}^{t}$. Hence $A$ depends on $\frac{k(k-1)}{2}+k(r-k)+(r-k)^{2}$ parameters, $B$ depends on $\frac{r(r+1)}{2}$ parameters and $C$ depends on $\frac{(r-k)(r-k+1)}{2}$ parameters. Then by (3.7) we get

$$
\operatorname{dim}(H)=\frac{k(k-1)}{2}+k(r-k)+(r-k)^{2}+\frac{r(r+1)}{2}+\frac{(r-k)(r-k+1)}{2}+1
$$

and

$$
\operatorname{dim}\left(Y_{k}\right)=\operatorname{dim}(S p(2 r))-\operatorname{dim}(H)=r(2 r+1)-\operatorname{dim}(H)
$$

yields the formula in the statement.

Finally, consider the case $k=2 r$, and let $H \subset S p(2 r)$ be the stabilizer of the identity matrix. The equality

$$
\left(\begin{array}{cc}
A & B \\
C & D
\end{array}\right)\left(\begin{array}{cc}
I_{r, r} & 0_{r, r} \\
0_{r, r} & I_{r, r}
\end{array}\right)\left(\begin{array}{cc}
A^{t} & C^{t} \\
B^{t} & D^{t}
\end{array}\right)=\left(\begin{array}{cc}
A A^{t}+B B^{t} & A C^{t}+B D^{t} \\
C A^{t}+D B^{t} & C C^{t}+D D^{t}
\end{array}\right)=\left(\begin{array}{cc}
\lambda I_{r, r} & 0_{r, r} \\
0_{r, r} & \lambda I_{r, r}
\end{array}\right)
$$

for some $\lambda \in K^{*}$ yields, applying as usual the transformation (3.3), the following system of equations

$$
\begin{cases}-C^{t}\left(A-I_{r, r}\right)+\left(A-I_{r, r}\right)^{t} C & =0_{r, r} \\ -C^{t} B^{t}+\left(A-I_{r, r}\right)^{t}\left(D+I_{r, r}\right) & =\left(D-I_{r, r}\right)^{t}\left(A-I_{r, r}\right)-B^{t} C \\ -\left(D-I_{r, r}\right)^{t} B+B^{t}\left(D-I_{r, r}\right) & =0_{r, r} .\end{cases}
$$

Note that if $M \in H$ taking the determinants on both sides of $M^{T} \Omega M=\lambda \Omega$ we see that $\lambda$ can only take finitely many values. Hence, by Remark 3.2 we have the following relations for the tangent space of $H$ at the identity

$$
A=-D^{t}, B=B^{t}, C=C^{t}, C=-B^{t}, A=-A^{t}
$$

Therefore, the tangent space consists of matrices of the following form

$$
\left(\begin{array}{cc}
A & B \\
-B^{t} & -A^{t}
\end{array}\right)
$$

with $B=B^{t}$ and $A=-A^{t}$. We conclude that

$$
\operatorname{dim}(H)=\frac{r(r+1)}{2}+\frac{r(r-1)}{2}=r^{2}
$$

and hence $\operatorname{dim}\left(Y_{2 r}\right)=r(2 r+1)-r^{2}=r(r+1)$.

Corollary 3.8. The projective variety $X_{2 r}$ is irreducible and its dimension is given by $\operatorname{dim}\left(X_{2 r}\right)=r(r+1)$.

Proof. The variety $X_{2 r}$ is the closure of an $S p(2 r)$-orbit, so it is irreducible. Since $X_{2 r}=Y_{2 r}$ the formula for its dimension follows from Proposition 3.6 .

Example 3.9. Consider the case $r=1$. Then Corollary 3.8 yields $\operatorname{dim}\left(X_{2}\right)=2$ and hence $X_{2}=\mathbb{P}^{2}$. Moreover, $\mathrm{O}_{2}=\mathbb{P}^{2} \backslash C$ where $C \subset \mathbb{P}^{2}$ is the conic parametrizing rank one matrices.

Remark 3.10. We work out equations for $X_{2 r}$. The points of the orbit $O_{2 r}$ represent symmetric matrices having a scalar multiple that is symplectic, that is $Z^{t} \Omega Z=\lambda \Omega$ for some $\lambda \in K^{*}$. The matrix $N=Z^{t} \Omega Z$ is skew-symmetric and so $N_{i, i}=0$ for $i=0, \ldots, 2 r-1$. Furthermore, for any $i=0, \ldots, 2 r-2$ we must have

$$
N_{i, i+1}=\cdots=N_{i, r+i-1}=N_{i, r+i+1}=\cdots=N_{i, 2 r-1}=0 .
$$

This gives $2 r-i-2$ quadratic equations for any $i=0, \ldots, r-1$, and $2 r-i-1$ quadratic equations for any $i=r, \ldots, 2 r-1$. Moreover, we must have

$$
N_{0, r}=N_{1, r+1}=\cdots=N_{r-1,2 r-1}
$$

and hence we get $r-1$ additional quadratic equations. Summing-up we get

$$
\sum_{i=0}^{r-1}(2 r-i-2)+\sum_{i=r}^{2 r-1}(2 r-i-1)+r-1=(2 r+1)(r-1)
$$

quadratic equations for $X_{2 r}$ in $\mathbb{P}^{N}$. 
Now, we explicitly compute these equations. Consider a general symmetric matrix $Z=\left(z_{i, j}\right)_{i, j=0, \ldots, 2 r-1}$ with $z_{i, j}=z_{j, i}$ and the standard symplectic form $\Omega$. Then

$$
c_{i, j}:=(Z \cdot \Omega)_{i, j}=\sum_{k=0}^{2 r-1} z_{i, k} \Omega_{k, j}= \begin{cases}z_{i, j-r} & \text { for } j \geqslant r \\ -z_{i, j+r} & \text { for } j<r\end{cases}
$$

and so

$$
N_{i, j}:=(Z \cdot \Omega \cdot Z)_{i, j}=\sum_{k=0}^{2 r-1} c_{i, k} z_{k, j}=\sum_{k=0}^{r-1} c_{i, k} z_{k, j}+\sum_{k=r}^{2 r-1} c_{i, k} z_{k, j}=\sum_{k=0}^{r-1}-z_{i, k+r} z_{k, j}+z_{i, k} z_{k+r, j}
$$

Summing-up, the equations

$$
\begin{cases}N_{l, r+l}-N_{l+1, r+l+1}=0 & \text { for } l=0, \ldots, r-2 \\ N_{i, j}=0 & \text { for } i=0, \ldots, 2 r-2, j>i, j \neq r+i\end{cases}
$$

can be explicitly written as follow

$$
\begin{cases}\sum_{k=0}^{r-1}-z_{l, k+r} z_{k, r+l}+z_{l, k} z_{k+r, r+l}+z_{l+1, k+r} z_{k, r+l+1}-z_{l+1, k} z_{k+r, r+l+1}=0 & \text { for } l=0, \ldots, r-2 \\ \sum_{k=0}^{r-1}-z_{i, k+r} z_{k, j}+z_{i, k} z_{k+r, j}=0 & \text { for } i=0, \ldots, 2 r-2, j>i, j \neq r+i .\end{cases}
$$

Now, our aim is to construct a wonderful compactification of the space of complete symmetric symplectic forms.

Construction 3.11. Set $S_{h}\left(\mathcal{V}_{2}^{2 r-1}\right):=\operatorname{Sec}_{h}\left(\mathcal{V}_{2}^{2 r-1}\right) \cap X_{2 r}$. Let us consider the following sequence of blow-ups:

- $X_{2 r}^{(1)}$ is the blow-up of $X_{2 r}^{(0)}:=X_{2 r}$ along the Veronese variety $\mathcal{V}_{2}^{2 r-1} \subset X_{2 r}$;

- $X_{2 r}^{(2)}$ is the blow-up of $X_{2 r}^{(1)}$ along the strict transform of $S_{2}\left(\mathcal{V}_{2}^{2 r-1}\right)$;

:

- $X_{2 r}^{(i)}$ is the blow-up of $X_{2 r}^{(i-1)}$ along the strict transform of $S_{i}\left(\mathcal{V}_{2}^{2 r-1}\right)$;

- $X_{2 r}^{(r-1)}$ is the blow-up of $X_{2 r}^{(r-2)}$ along the strict transform of $S_{r-1}\left(\mathcal{V}_{2}^{2 r-1}\right)$.

Let $f_{i}: X_{2 r}^{(i)} \rightarrow X_{2 r}^{(i-1)}$ be the blow-up morphism. We will denote by $E_{i}$ both the exceptional divisor of $f_{i}$ and its strict transforms in the subsequent blow-ups. We set $\mathcal{S}_{2 r}:=X_{2 r}^{(r-1)}$ and we indicate with $f: \mathcal{S}_{2 r} \rightarrow X_{2 r}$ the composition of the $f_{i}$.

Let $M_{2 r, 2 r}(K)$ be the space of $2 r \times 2 r$ matrices. Following dlCb16 we define the operator

$$
\begin{aligned}
& \Phi_{\Omega}: M_{2 r, 2 r}(K) \quad \longrightarrow \quad M_{2 r, 2 r}(K) \\
& A \quad \mapsto \quad \Omega^{-1} A^{T} \Omega
\end{aligned}
$$

Definition 3.12. A matrix $A \in M_{2 r, 2 r}(K)$ is symplectically congruent to a matrix $B \in M_{2 r, 2 r}(K)$ if there exists a symplectic matrix $Q$ such that $Q A Q^{T}=B$.

By [dCb16, Theorem 21] a matrix $A \in M_{2 r, 2 r}(K)$ is symplectically congruent to a diagonal matrix if and only if $A$ is symmetric and $A \Phi_{\Omega}(A)$ is diagonalizable.

Proposition 3.13. The quadratic equations in Remark 3.10 cut out $X_{2 r}$ set-theoretically. Furthermore $Y_{i}=$ $\operatorname{Sec}_{i}\left(\mathcal{V}_{2}^{2 r-1}\right) \cap X_{2 r}$, set-theoretically, and there is a stratification

$$
Y_{1} \subset Y_{2} \subset \cdots \subset Y_{r-1} \subset Y_{r} \subset Y_{2 r}=X_{2 r} .
$$

In particular, $\operatorname{dim}\left(\operatorname{Sec}_{i}\left(\mathcal{V}_{2}^{2 r-1}\right) \cap X_{2 r}\right)=2 r i+i-i^{2}-1$ for $i=1, \ldots, r$ and $Y_{r}$ is a divisor in $X_{2 r}$.

Proof. Let $Z$ be a symmetric matrix satisfying the equations in Remark 3.10. Then we have two cases:

(i) $N_{0, r}=\cdots=N_{r-1,2 r-1}=\lambda \in K^{*}$;

(ii) $N_{0, r}=\cdots=N_{r-1,2 r-1}=0$.

Consider (i). Then $Z^{t} \Omega Z=\lambda \Omega$ and $\operatorname{det}(Z) \neq 0$. Moreover,

$$
Z \Phi_{\Omega}(Z)=Z \Omega^{-1} Z^{t} \Omega=-Z^{t} \Omega Z \Omega=-\lambda \Omega^{2}=\lambda I_{2 r, 2 r}
$$

and by [lCb16, Theorem 21] $Z$ is symplectically congruent to a diagonal matrix.

In case (ii) $Z^{t} \Omega Z$ is the zero matrix. So $\operatorname{det}(Z)=0$, and $Z \Phi_{\Omega}(Z)$ is the zero matrix as well. Again, dlCb16, Theorem 21 ] yields that $Z$ is symplectically congruent to a diagonal matrix.

So if $Z$ is a symmetric matrix satisfying the equations in Remark 3.10 there is a symplectic matrix $Q$ such that $Q Z Q^{t}=D$ with $D$ diagonal. Our aim is to prove that $D$ can be moved to a matrix of the form $I_{k}$, where $k$ is the rank of $D$, with the action of the symplectic group. 
Let $D_{\alpha}=\operatorname{diag}\left(\alpha_{1}, \ldots, \alpha_{2 r}\right)$ be a diagonal matrix satisfying the equations in Remark 3.10. Then either $\alpha_{i} \alpha_{r+i}=0$ for all $i=1, \ldots, r$, or $\alpha_{i} \alpha_{r+i}=\lambda \in K^{*}$ for all $i=1, \ldots, r$. Write

$$
D_{\alpha}=\left(\begin{array}{cc}
D_{\alpha_{1}, \ldots, \alpha_{p}} & 0_{r, r} \\
0_{r, r} & D_{\alpha_{p+1}, \ldots, \alpha_{p+q}}
\end{array}\right)
$$

with $p+q \leqslant r$, where $D_{\alpha_{1}, \ldots, \alpha_{p}}$ is an $r \times r$ diagonal matrix with the $\alpha_{i}$ appearing on the diagonal, and similarly for $D_{\alpha_{p+1}, \ldots, \alpha_{p+q}}$. Note that up to permuting the upper and lower diagonal simultaneously we may assume that $\alpha_{1}, \ldots, \alpha_{p}$ are the first $p$ entries on the diagonal of $D_{\alpha_{1}, \ldots, \alpha_{p}}$, and $\alpha_{p+1}, \ldots, \alpha_{p+q}$ are the last $q$ entries on the diagonal of $D_{\alpha_{p+1}, \ldots, \alpha_{p+q}}$.

Now, set $p+q=r$. Let $A, B, C, D$ be $r \times r$ matrices defined as follows:

- the first $p$ entries on the diagonal of $A$ are $a_{i} \in K^{*}$ for $i=1, \ldots, p$, and the other entries are zero;

- the last $q$ entries on the diagonal of $B$ are $-b_{i}^{-1} \in K^{*}$ for $i=p+1, \ldots, p+q$, and the other entries are zero;

- the last $q$ entries on the diagonal of $C$ are $b_{i} \in K^{*}$ for $i=p+1, \ldots, p+q$, and the other entries are zero;

- the first $p$ entries on the diagonal of $D$ are $a_{i}^{-1} \in K^{*}$ for $i=1, \ldots, p$, and the other entries are zero.

Consider the matrix

$$
P=\left(\begin{array}{cc}
A & B \\
C & D
\end{array}\right)
$$

and note that $P$ is symplectic. Furthermore, by taking $a_{i}, b_{j}$ such that $a_{i}^{2}=\alpha_{i}$ for $i=1, \ldots, p$, and $b_{j}^{-2}=\alpha_{j}$ for $j=p+1, \ldots, p+q$ we have $P^{t} I_{r} P=D_{\alpha}$ when $p+q=r$.

If $p+q<r$ by permuting the upper diagonal of $I_{p+q}$, we transform $I_{p+q}$ into the matrix $I_{p+q}^{*}$ whose entries on the diagonal are $\left(I_{p+q}^{*}\right)_{i, i}=1$ for $i=1, \ldots, p,\left(I_{p+q}^{*}\right)_{i, i}=0$ for $i=p+1, \ldots, p+s,\left(I_{p+q}^{*}\right)_{i, i}=1$ for $i=$ $p+s+1, \ldots, p+s+q$, and $\left(I_{p+q}^{*}\right)_{i, i}=0$ for $i=p+s+q+1, \ldots, 2 r$, where $p+s+q=r$. In this case consider $r \times r$ diagonal matrices $\bar{A}, \bar{B}, \bar{C}, \bar{D}$ such that

- the first $p$ entries on the diagonal of $\bar{A}$ are $a_{i} \in K^{*}$ for $i=1, \ldots, p,(\bar{A})_{i, i}=1$ for $i=p+1, \ldots, p+s,(\bar{A})_{i, i}=0$ for $i=p+s+1, \ldots, p+s+q$;

- the first $p+s$ entries on the diagonal of $\bar{B}$ are zero, followed by $-b_{p+1}^{-1}, \ldots,-b_{p+q}^{-1}$;

- the first $p+s$ entries on the diagonal of $\bar{C}$ are zero, followed by $b_{p+1}, \ldots, b_{p+q}$;

- the first $p$ entries on the diagonal of $\bar{D}$ are $a_{i}^{-1} \in K^{*}$ for $i=1, \ldots, p,(\bar{D})_{i, i}=1$ for $i=p+1, \ldots, p+s$, $(\bar{D})_{i, i}=0$ for $i=p+s+1, \ldots, p+s+q$;

and set

$$
\bar{P}=\left(\begin{array}{cc}
\bar{A} & \bar{B} \\
\bar{C} & \bar{D}
\end{array}\right)
$$

Then $\bar{P}$ is symplectic and by taking again $a_{i}, b_{j}$ such that $a_{i}^{2}=\alpha_{i}$ for $i=1, \ldots, p$, and $b_{j}^{-2}=\alpha_{j}$ for $j=p+1, \ldots, p+q$ it holds $\bar{P}^{t} I_{p+q}^{*} \bar{P}=D_{\alpha}$.

Furthermore, when $D_{\alpha}$ is of maximal rank we consider the diagonal symplectic matrix

$$
P=\operatorname{diag}\left(a_{1}, \ldots, a_{r}, a_{1}^{-1}, \ldots, a_{r}^{-1}\right) .
$$

Note that taking $a_{i} \in K^{*}$ such that $a_{i}^{2}=\alpha_{i} \mu^{-1}$, with $\mu^{2}=\lambda$, for $i=1, \ldots, r$, we get that $P^{t} I_{2 r, 2 r} P$ is a scalar multiple of $D_{\alpha}$. Consider the matrices

$$
\Psi_{t}=\left(\begin{array}{cc}
I_{r, r} & 0_{r, r} \\
0_{r, r} & T_{r, r}
\end{array}\right) ; \quad \Lambda_{t}=\left(\begin{array}{ccc}
I_{k, k} & 0 & 0_{k, 2 r-k-1} \\
0 & t & 0_{1,2 r-k-1} \\
0_{2 r-k-1, k} & 0_{2 r-k-1,1} & 0_{2 r-k-1,2 r-k-1}
\end{array}\right) \quad \text { for } k=1, \ldots, r-1
$$

where $T_{r, r}=\operatorname{diag}(t, \ldots, t)$. By the first part of the proof we have that $\left\{\Psi_{t}\right\}_{t \in K^{*}}$ is a family of matrices in $O_{2 r}$, and $\lim _{t \mapsto 0} \Psi_{t}=I_{r}$. Furthermore, $\left\{\Lambda_{t}\right\}_{t \in K^{*}}$ is a family of matrices in $O_{k+1}$, and $\lim _{t \mapsto 0} \Lambda_{t}=I_{k}$ for $k=1, \ldots, r-1$.

Summing-up we proved that if $Z$ is a symmetric matrix for rank $k$ with $1 \leqslant k \leqslant r$ or $k=2 r$ satisfying the equations in Remark 3.10 then $Z$ can be symplectically transformed into the matrix $I_{k}$, and hence it lies in $O_{k}$.

Remark 3.14. Proposition 3.13 yields that the Veronese variety $\mathcal{V}_{2}^{2 r-1}$ is contained in $X_{2 r}$. On the other hand, for $h \geqslant 2$ the secant variety $\operatorname{Sec}_{h}\left(\mathcal{V}_{2}^{2 r-1}\right)$ is not contained in $X_{2 r}$.

Proposition 3.15. For any $k \geqslant r$ we have that $X_{2 r} \cap \operatorname{Sec}_{r}\left(\mathcal{V}_{2}^{2 r-1}\right)=X_{2 r} \cap \operatorname{Sec}_{k}\left(\mathcal{V}_{2}^{2 r-1}\right)$

Proof. Assume there is a matrix $M \in X_{2 r} \cap \operatorname{Sec}_{k}\left(\mathcal{V}_{2}^{2 r-1}\right)$ of rank $r<k<2 r-1$. Arguing as in the proof of Proposition 3.13 we can move $M$ with the action of $S p(2 r)$ in a diagonal matrix $D_{k}$ of rank $k$, and $D_{k} \in$ $X_{2 r} \cap \operatorname{Sec} k\left(\mathcal{V}_{2}^{2 r-1}\right)$. However, $D_{k}$ does not satisfy the equation $N_{j, r+j}-N_{j+1, r+j+1}=0$ for $X_{2 r}$ in $\operatorname{Remark} 3.10$. A contradiction. 
We analyze in detail the geometry of the objects we introduced in the first non trivial case, namely $r=2$.

Proposition 3.16. The variety $X_{4}$ is isomorphic to the Grassmannian $\mathbb{G}(1,4) \subset \mathbb{P}^{9}$ of lines in $\mathbb{P}^{4}$. Furthermore, $\mathcal{V}_{2}^{3} \subset X_{4}$, and $S_{2}\left(\mathcal{V}_{2}^{3}\right) \subset X_{4}$ is an irreducible and reduced divisor singular along $\mathcal{V}_{2}^{3}$. In particular, the equations in Remark 3.10 cut out $X_{4}$ scheme-theoretically, and $S_{2}\left(\mathcal{V}_{2}^{3}\right)=Y_{2}$ scheme-theoretically.

Proof. Consider homogeneous coordinates $z_{i, j}$ on $\mathbb{P}^{9}$ and identify them with the entries of a general $4 \times 4$ symmetric matrix $Z$. The change of variables $z_{0,0} \mapsto z_{1,2}, z_{0,1} \mapsto z_{0,1}, z_{0,2} \mapsto \frac{z_{1,4}-z_{2,3}}{2}, z_{0,3} \mapsto z_{0,2}, z_{1,1} \mapsto z_{1,3}, z_{1,2} \mapsto$ $z_{0,3}, z_{1,3} \mapsto \frac{z_{1,4}+z_{2,3}}{2}, z_{2,2} \mapsto z_{3,4}, z_{2,3} \mapsto z_{0,4}, z_{3,3} \mapsto z_{2,4}$ transforms the five equations in Remark 3.10 into the standard Plücker equations cutting out $\mathbb{G}(1,4)$ in $\mathbb{P}^{9}$.

By Remark 3.14 we have $\mathcal{V}_{2}^{3} \subset X_{4}$. We can compute the tangent cones of $S_{2}\left(\mathcal{V}_{2}^{3}\right) \subset X_{4}$ at a point representing a rank one matrix, and at a point representing a rank two matrix using the equations for $X_{4}$ in Remark 3.10 together with the equations cutting out $\operatorname{Sec}_{2}\left(\mathcal{V}_{2}^{3}\right)$. In the first case we get a cone with a 3 -dimensional vertex over $\mathcal{V}_{1}^{2}$ which in particular is irreducible and reduced, and in the second case we get a 5-dimensional linear space. Finally, since by Proposition $3.13 Y_{2}$ has dimension five we conclude that the equations in Remark 3.10 together with the equations cutting out $\operatorname{Sec}_{2}\left(\mathcal{V}_{2}^{3}\right)$ define $Y_{2}$ scheme-theoretically.

Remark 3.17. The variety $X_{4} \cong \mathbb{G}(1,4)$ has been studied in relation to moduli spaces of rank two vector bundles over a smooth quadric OS94, Table I].

Proposition 3.18. The tangent cone $T C_{p_{k}}\left(X_{2 r}\right)$ of $X_{2 r}$ at a point $p_{k} \in S_{k}\left(\mathcal{V}_{2}^{2 r-1}\right) \backslash S_{k-1}\left(\mathcal{V}_{2}^{2 r-1}\right)$ for $k=1, \ldots, r-1$ is a cone with vertex of dimension $k(2 r+1-k)-1$ over $X_{2(r-k)}$. Moreover, $X_{2 r}$ is smooth along $S_{r}\left(\mathcal{V}_{2}^{2 r-1}\right) \backslash S_{r-2}\left(\mathcal{V}_{2}^{2 r-1}\right)$, and the equations in Remark 3.10 define $X_{2 r}$ scheme-theoretically.

The tangent cone $T C_{p_{k}}\left(S_{h}\left(\mathcal{V}_{2}^{2 r-1}\right)\right)$ of $S_{h}\left(\mathcal{V}_{2}^{2 r-1}\right)$ at a point $p_{k} \in S_{k}\left(\mathcal{V}_{2}^{2 r-1}\right) \backslash S_{k-1}\left(\mathcal{V}_{2}^{2 r-1}\right)$ for $k=1, \ldots, r-1, k<$ $h$ is a cone with vertex of dimension $k(2 r+1-k)-1$ over $S_{h-k}\left(\mathcal{V}_{2}^{2(r-k)-1}\right)$. Moreover, the equations in Remark 3.10 together with the equations cutting out $\operatorname{Sec}_{h}\left(\mathcal{V}_{2}^{2 r-1}\right)$ define $S_{h}\left(\mathcal{V}_{2}^{2 r-1}\right)$ scheme-theoretically.

In particular, $X_{2 r}$ is smooth along $S_{r}\left(\mathcal{V}_{2}^{2 r-1}\right) \backslash S_{r-2}\left(\mathcal{V}_{2}^{2 r-1}\right)$ and $S_{r}\left(\mathcal{V}_{2}^{2 r-1}\right)$ is a divisor in $X_{2 r}$.

Proof. Let $p_{k}=\left(p_{i, j}\right)_{i, j=0, \ldots, 2 r-1, i \leqslant j}$ be the point representing the standard matrix of rank $k$ with $p_{i, i}=1$ for $i=0, \ldots, k-1$ and $p_{i, j}=0$ otherwise.

We proceed by induction on $r$. The base case $r=2$ is in Proposition 3.16. We will use the equations in Remark 3.10 to compute $T C_{p_{k}} X_{2 r}$. Consider the change of coordinates $z_{i, i} \mapsto z_{i, i}-z_{0,0}$ for $i=1, \ldots, k-1$, and set $z_{0,0}=1$. Note that the lowest degree terms of the equations in Remark 3.10 after this change of coordinates are obtained by removing from $Z^{t} \Omega Z=\lambda \Omega$ the rows and columns indexed by $0, \ldots, k-1, r, \ldots, r+k-1$. Therefore, we get a cone with vertex of dimension $k(2 r+1-k)-1$ over $X_{2(r-k)}$ which by induction hypothesis is irreducible and reduced since the equations in Remark 3.10 define $X_{2(r-k)}$ scheme-theoretically. Now, $k(2 r+1-k)+\operatorname{dim}\left(X_{2(r-k)}\right)=$ $\operatorname{dim}\left(X_{2 r}\right)$ yields that this is the tangent cone $T C_{p_{k}} X_{2 r}$, and hence the equations in Remark 3.10 define $X_{2 r}$ scheme-theoretically. Note that at the points representing $I_{r}$ and $I_{2 r, 2 r}$ the equations in Remark 3.10 yield a linear subspace of the same dimension of $X_{2 r}$.

Now, consider $S_{h}\left(\mathcal{V}_{2}^{2 r-1}\right)$. Note that $T C_{p_{k}} S_{h}\left(\mathcal{V}_{2}^{2 r-1}\right)$ is contained in $T C_{p_{k}} X_{2 r} \cap T C_{p_{k}} \operatorname{Sec}_{h}\left(\mathcal{V}_{2}^{2 r-1}\right)$. By the previous computation of $T C_{p_{k}} X_{2 r}$ and the computation of $T C_{p_{k}} \operatorname{Sec}_{h}\left(\mathcal{V}_{2}^{2 r-1}\right)$ in Proposition [2.7 we conclude that $T C_{p_{k}} X_{2 r} \cap T C_{p_{k}} \operatorname{Sec}_{h}\left(\mathcal{V}_{2}^{2 r-1}\right)$ is a cone with vertex of dimension $k(2 r+1-k)-1$ over $S_{h}\left(\mathcal{V}_{2}^{2(r-k)-1}\right)=$ $\operatorname{Sec}_{h-k}\left(\mathcal{V}_{2}^{2(r-k)-1}\right) \cap X_{2(r-k)}$. Again by induction this is an irreducible and reduced cone which by the computation of the dimension of $S_{h}\left(\mathcal{V}_{2}^{2 r-1}\right)$ in Proposition 3.13 must coincide with $T C_{p_{k}} S_{h}$. Hence the equations in Remark 3.10 together with the equations cutting out $\operatorname{Sec}_{h}\left(\mathcal{V}_{2}^{2 r-1}\right)$ define $S_{h}\left(\mathcal{V}_{2}^{2 r-1}\right)$ scheme-theoretically.

Now, we are ready to prove the main result of this section. We will denote by $S_{h}^{(i)}\left(\mathcal{V}_{2}^{2 r-1}\right)$ the strict transform of $S_{h}\left(\mathcal{V}_{2}^{2 r-1}\right)$ in $X_{2 r}^{(i)}$.

Theorem 3.19. For any $i=1, \ldots, r-1$ the strict transform $S_{i+1}^{(i)}\left(\mathcal{V}_{2}^{2 r-1}\right)$ of $S_{i+1}\left(\mathcal{V}_{2}^{2 r-1}\right)$ in $X_{2 r}^{(i)}$ is smooth. Moreover, the variety $\mathcal{S}_{2 r}$ is smooth and the exceptional divisors $E_{1}, \ldots, E_{r-1} \subset \mathcal{S}_{2 r}$ are smooth as well.

The closures of the orbits of the $S p(2 r)$-action on $\mathcal{S}_{2 r}$ induced by the action in (3.5) are given by all the possible intersections among $E_{1}, \ldots, E_{r-1}, S_{r}^{(r-1)}\left(\mathcal{V}_{2}^{2 r-1}\right)$ and $X_{2 r}^{(i)}$ itself.

In particular, the variety $\mathcal{S}_{2 r}$ with boundary divisors $E_{1}, \ldots, E_{r-1}, S_{r}^{(r-1)}\left(\mathcal{V}_{2}^{2 r-1}\right)$ is wonderful.

Proof. For every $r$ in $X_{2 r}^{(0)}$ we have $S_{1}^{(0)}\left(\mathcal{V}_{2}^{2 r-1}\right)=\mathcal{V}_{2}^{2 r-1}$ which is smooth. We will assume that $S_{j}^{(j-1)}\left(\mathcal{V}_{2}^{2 r-1}\right)$ is smooth for every $r$ and for every $j<i$ and prove that also $S_{i}^{(i-1)}\left(\mathcal{V}_{2}^{2 r-1}\right)$ in $X_{2 r}^{(i-1)}$ is smooth. We have 
$S_{i}^{(i-1)}\left(\mathcal{V}_{2}^{2 r-1}\right)=\operatorname{Sec}_{i}^{(i-1)}\left(\mathcal{V}_{2}^{2 r-1}\right) \cap X_{2 r}^{(i-1)}$, so we consider $S_{i}^{(i-1)}\left(\mathcal{V}_{2}^{2 r-1}\right)$ inside $\mathcal{Q}(2 r-1)_{(i-1)}$. By Proposition 2.10, for every $r$ and for every $i=0, \ldots, 2 r-1$ the varieties $\mathcal{Q}(2 r-1)_{(i-1)}, \operatorname{Sec}_{i}^{(i-1)}\left(\mathcal{V}_{2}^{2 r-1}\right), E_{1}^{q}, \ldots, E_{i-1}^{q}$ are smooth.

Now, $S_{i}^{(i-1)}\left(\mathcal{V}_{2}^{2 r-1}\right)$ is smooth away from $E_{1}^{q}, \ldots, E_{i-1}^{q}$. Moreover, by Proposition 3.18 for every $k=1, \ldots, i-1$, $S_{i}^{(i-1)}\left(\mathcal{V}_{2}^{2 r-1}\right) \cap E_{k}^{q} \rightarrow S_{k}^{(k-1)}\left(\mathcal{V}_{2}^{2 r-1}\right)$ is a fibration with fibers isomorphic to $S_{i-k}^{(i-k-1)}\left(\mathcal{V}_{2}^{2(r-k)-1}\right)$ which is smooth by induction. Proposition 2.8 yields that $S_{i}^{(i-1)}\left(\mathcal{V}_{2}^{2 r-1}\right) \cap E_{k}^{q}$ is smooth for $k=1, \ldots, i-1$. Now, since by Proposition 3.13 we have

$$
\operatorname{dim} S_{k}^{(k-1)}\left(\mathcal{V}_{2}^{2 r-1}\right)+\operatorname{dim} S_{i-k}^{(i-k-1)}\left(\mathcal{V}_{2}^{2(r-k)-1}\right)=2 r i+i-i^{2}-2=\operatorname{dim} S_{i}^{(i-1)}\left(\mathcal{V}_{2}^{2 r-1}\right)-1
$$

we get that $S_{i}^{(i-1)}\left(\mathcal{V}_{2}^{2 r-1}\right)$ is smooth as well.

By Proposition 3.18 for every $r$, in $X_{2 r}^{(1)}$ we have that $E_{1} \cap S_{2}^{(1)}\left(\mathcal{V}_{2}^{2 r-1}\right) \rightarrow \mathcal{V}_{2}^{2 r-1}$ is a fibration with fibers isomorphic to $\mathcal{V}_{2}^{2(r-1)-1}$ and then by Proposition 2.8 $E_{1} \cap S_{2}^{(1)}\left(\mathcal{V}_{2}^{2 r-1}\right)$ is smooth of dimension $4 r-4=\operatorname{dim}\left(S_{2}^{(1)}\left(\mathcal{V}_{2}^{2 r-1}\right)\right)-1$. More generally, consider intersections of the form $S_{i+1}^{(i)}\left(\mathcal{V}_{2}^{2 r-1}\right) \cap E_{j_{1}} \cap \cdots \cap E_{j_{t}}$, for $1 \leqslant j_{1}<\cdots<j_{t} \leqslant i$. By Proposition 3.18, the restriction of the blow-down morphism

$$
S_{i+1}^{(i)}\left(\mathcal{V}_{2}^{2 r-1}\right) \cap E_{j_{1}} \cap \cdots \cap E_{j_{t}} \rightarrow E_{j_{1}} \cap \cdots \cap E_{j_{t-1}} \cap S_{j_{t}}^{\left(j_{t}-1\right)}\left(\mathcal{V}_{2}^{2 r-1}\right)
$$

has fibers isomorphic to $S_{i+1-j_{t}}^{\left(i-j_{t}\right)}\left(\mathcal{V}_{2}^{2\left(r-j_{t}\right)-1}\right)$. Now, by Proposition $2.8 S_{i+1}^{(i)}\left(\mathcal{V}_{2}^{2 r-1}\right) \cap E_{j_{1}} \cap \cdots \cap E_{j_{t}}$ is smooth since we proved before that $S_{i+1-j_{t}}^{\left(i-j_{t}\right)}\left(\mathcal{V}_{2}^{2\left(r-j_{t}\right)-1}\right)$ is smooth and $E_{j_{1}} \cap \cdots \cap E_{j_{t-1}} \cap S_{j_{t}}^{\left(j_{t}-1\right)}\left(\mathcal{V}_{2}^{2 r-1}\right)$ is smooth by induction. Moreover,

$$
\operatorname{dim}\left(S_{i+1}^{(i)}\left(\mathcal{V}_{2}^{2 r-1}\right) \cap E_{j_{1}} \cap \cdots \cap E_{j_{t}}\right)=\operatorname{dim}\left(E_{j_{1}} \cap \cdots \cap E_{j_{t-1}} \cap S_{j_{t}}^{\left(j_{t}-1\right)}\left(\mathcal{V}_{2}^{2 r-1}\right)\right)+\operatorname{dim}\left(S_{i+1-j_{t}}^{\left(i-j_{t}\right)}\left(\mathcal{V}_{2}^{2\left(r-j_{t}\right)-1}\right)\right)
$$

and by induction $\operatorname{dim}\left(E_{j_{1}} \cap \cdots \cap E_{j_{t-1}} \cap S_{j_{t}}^{\left(j_{t}-1\right)}\left(\mathcal{V}_{2}^{2 r-1}\right)\right)=2 r j_{t}+j_{t}-j_{t}^{2}-1-(t-1)$. This yields, using Proposition 3.13, that

$$
\operatorname{dim}\left(S_{i+1}^{(i)}\left(\mathcal{V}_{2}^{2 r-1}\right) \cap E_{j_{1}} \cap \cdots \cap E_{j_{t}}\right)=2 r(i+1)+(i+1)-(i+1)^{2}-1-t=\operatorname{dim}\left(S_{i+1}^{(i)}\left(\mathcal{V}_{2}^{2 r-1}\right)\right)-t .
$$

Now, consider the variety $\mathcal{S}_{2 r}$ as a subvariety of the variety $\mathcal{Q}(2 r-1)_{r-1}$ in Construction 2.5. By Proposition $3.18 \mathcal{S}_{2 r}$ is smooth away from the exceptional divisors. Furthermore, the exceptional divisor $E_{i}^{q}$ in Construction 2.5 intersects $\mathcal{S}_{2 r}$ in the exceptional divisor $E_{i}$ in Construction 3.11 By Proposition $3.18 E_{i} \rightarrow S_{i}^{(i-1)}\left(\mathcal{V}_{2}^{2 r-1}\right)$ is a fibration with $\mathcal{S}_{2(r-i)}$ as fiber. Hence $E_{i}^{q} \cap \mathcal{S}_{2 r}$ is a smooth divisor in $\mathcal{S}_{2 r}$ and therefore $\mathcal{S}_{2 r}$ is smooth.

Now, consider an intersection of the form $E_{j_{1}} \cap \cdots \cap E_{j_{t}}$ and the fibration

$$
E_{j_{1}} \cap \cdots \cap E_{j_{t}} \rightarrow E_{j_{1}} \cap \ldots E_{j_{t-1}} \cap S_{j_{t}}^{\left(j_{t}-1\right)}\left(\mathcal{V}_{2}^{2 r-1}\right) .
$$

By Proposition 3.18 this fibration has fibers isomorphic to $\mathcal{S}_{2\left(r-j_{t}\right)}$. By the previous part of the proof we have that

$$
\operatorname{dim}\left(E_{j_{1}} \cap \ldots E_{j_{t-1}} \cap S_{j_{t}}^{\left(j_{t}-1\right)}\left(\mathcal{V}_{2}^{2 r-1}\right)\right)+\operatorname{dim}\left(\mathcal{S}_{2\left(r-j_{t}\right)}\right)=r^{2}+r-t=\operatorname{dim}\left(\mathcal{S}_{2 r}\right)-t
$$

and hence the intersection $E_{j_{1}} \cap \cdots \cap E_{j_{t}}$ is transversal. Note also that considering the fibration

$$
S_{r}^{(r-1)}\left(\mathcal{V}_{2}^{2 r-1}\right) \cap E_{j_{1}} \cap \cdots \cap E_{j_{t}} \rightarrow E_{j_{1}} \cap \cdots \cap E_{j_{t-1}} \cap S_{j_{t}}^{\left(j_{t}-1\right)}\left(\mathcal{V}_{2}^{2 r-1}\right)
$$

and (3.20) we get that the intersection $S_{r}^{(r-1)}\left(\mathcal{V}_{2}^{2 r-1}\right) \cap E_{j_{1}} \cap \cdots \cap E_{j_{t}}$ is transversal as well.

Finally, for the claim about the orbit closures it is enough to recall that the $S p(2 r)$-action on $\mathcal{S}_{2 r}$ is the restriction of the $S L(2 r)$-action on $\mathcal{Q}(2 r-1)_{r-1}$ in (2.2) and to use the statement about the orbit closures in Proposition 2.10 .

Proposition 3.21. We have that

$$
\text { mult }_{S_{r}\left(\mathcal{V}_{2}^{2 r-1}\right)} S_{2 r-1}\left(\mathcal{V}_{2}^{2 r-1}\right)=r .
$$

Moreover, if $H_{X_{2 r}}$ is the hyperplane section of $X_{2 r}$, we have that $S_{r}\left(\mathcal{V}_{2}^{2 r-1}\right) \sim 2 H_{X_{2 r}}$.

Proof. We will compute the tangent cone of $S_{r}\left(\mathcal{V}_{2}^{2 r-1}\right)$ at the point $p_{r}=\left(p_{i, j}\right)_{i, j=0, \ldots, 2 r-1}$, where $p_{i, i}=1$ for $i=0, \ldots, r-1$ and $p_{i, j}=0$ otherwise.

Consider the change of coordinates $z_{i, i} \mapsto z_{i, i}-z_{0,0}$ ans set $z_{0,0}=1$. By Remark 3.10 the tangent space of $X_{2 r}$ at $p_{r}$ is cut out by a set of linear equations and among these equations we have $\left\{z_{i, j}=0\right\}$ for $i, j=r, \ldots, 2 r-2$, and $z_{i, i}=z_{i+1, i+1}$ for $i=r, \ldots, 2 r-2$.

Now, the tangent cone of $\operatorname{Sec}_{2 r-1}\left(\mathcal{V}_{2}^{2 r-1}\right)$ is cut out by the determinant of the bottom right $r \times r$ submatrix of the matrix $Z$ in (2.1). Note that substituting the relations on the $z_{i, j}$ above in this determinant we get $z_{2 r-1,2 r-1}^{r}$.

By Proposition $3.15 \operatorname{Sec}_{2 r-1}\left(\mathcal{V}_{2}^{2 r-1}\right)$ and $\operatorname{Sec}_{r}\left(\mathcal{V}_{2}^{2 r-1}\right)$ cut out on $X_{2 r}$ the same divisor set-theoretically. The previous computation yields that $\operatorname{Sec}_{2 r-1}\left(\mathcal{V}_{2}^{2 r-1}\right)$ cuts out $\operatorname{Sec}_{r}\left(\mathcal{V}_{2}^{2 r-1}\right) \cap X_{2 r}$ on $X_{2 r}$ with multiplicity $r$. 
Now, recall that by Remark 2.6] $\operatorname{deg}\left(\operatorname{Sec}_{2 r-1}\left(\mathcal{V}_{2}^{2 r-1}\right)\right)=2 r$. Let $D$ be the divisor $\operatorname{Sec}_{r}\left(\mathcal{V}_{2}^{2 r-1}\right) \cap X_{2 r}$. Finally $\operatorname{Sec}_{2 r-1}\left(\mathcal{V}_{2}^{2 r-1}\right) \cap X_{2 r} \sim 2 r H_{X_{2 r}}$ yields $D \sim 2 H_{X_{2 r}}$.

Remark 3.22. In the case $r=2$ we worked out explicitly the quadratic polynomial cutting out $S_{2}\left(\mathcal{V}_{2}^{3}\right)$ in $X_{4}$ and we got that $S_{2}\left(\mathcal{V}_{2}^{3}\right)=X_{4} \cap\left\{z_{0,3} z_{1,2}+z_{1,3}^{2}-z_{0,1} z_{2,3}-z_{1,1} z_{3,3}=0\right\}$.

\section{DivisORS ON $\mathcal{S}_{2 r}$}

Let $X$ be a normal projective $\mathbb{Q}$-factorial variety over an algebraically closed field of characteristic zero. We denote by $N^{1}(X)$ the real vector space of $\mathbb{R}$-Cartier divisors modulo numerical equivalence. The nef cone of $X$ is the closed convex cone $\operatorname{Nef}(X) \subset N^{1}(X)$ generated by classes of nef divisors.

The stable base locus $\mathbf{B}(D)$ of a $\mathbb{Q}$-divisor $D$ is the set-theoretic intersection of the base loci of the complete linear systems $|s D|$ for all positive integers $s$ such that $s D$ is integral

$$
\mathbf{B}(D)=\bigcap_{s>0} B(s D) \text {. }
$$

The movable cone of $X$ is the convex cone $\operatorname{Mov}(X) \subset N^{1}(X)$ generated by classes of movable divisors. These are Cartier divisors whose stable base locus has codimension at least two in $X$. The effective cone of $X$ is the convex cone $\operatorname{Eff}(X) \subset N^{1}(X)$ generated by classes of effective divisors. We have inclusions $\operatorname{Nef}(X) \subset \overline{\operatorname{Mov}(X)} \subset \overline{\operatorname{Eff}(X)}$. We refer to [Deb01, Chapter 1] for a comprehensive treatment of these topics.

In this section we will study the Picard rank and the cones of effective and nef divisors of the wonderful compactification $\mathcal{S}_{2 r}$. We will need the following result.

Lemma 4.2. Let $S O(2 r)$ be the special orthogonal group. Then

$$
S O(2 r) \cap S p(2 r) \cong G L(r) .
$$

In particular, $S O(2) \cong G L(1) \cong K^{*}$.

Proof. Consider the bilinear symmetric form given by the matrix $J=\left(\begin{array}{cc}0_{r, r} & I_{r, r} \\ I_{r, r} & 0_{r, r}\end{array}\right)$. Set $N=\left(\begin{array}{cc}I_{r, r} & \xi I_{r, r} \\ \frac{1}{2} I_{r, r} & -\frac{\xi}{2} I_{r, r}\end{array}\right)$, with $\xi^{2}=-1$. Note that $N^{t} J N=I_{2 r, 2 r}$ and $N^{t} \Omega N=-\xi \Omega$. Therefore, we may prove the statement for the intersection $S O_{J}(2 r) \cap S p(2 r)$, where $S O_{J}(2 r)$ is the group of determinant one matrices which are orthogonal with respect to $J$.

Let $M=\left(\begin{array}{cc}A & B \\ C & D\end{array}\right) \in G L(2 r)$ be a general $2 r \times 2 r$ invertible matrix, where $A, B, C, D$ are $r \times r$ matrices. Now, $M \in S O_{J}(2 r) \cap S p(2 r)$ if and only if

$$
\left\{\begin{array} { l } 
{ A ^ { t } C = 0 _ { r , r } ; } \\
{ A ^ { t } D = I _ { r , r } ; } \\
{ B ^ { t } C = 0 _ { r , r } ; } \\
{ B ^ { t } D = 0 _ { r , r } ; }
\end{array} \quad \text { that is } \quad \left\{\begin{array}{l}
D=A^{-t} \\
C=0_{r, r} \\
B=0_{r, r}
\end{array}\right.\right.
$$

and hence

$$
S O(2 r) \cap S p(2 r) \cong S O_{J}(2 r) \cap S p(2 r)=\left\{\left(\begin{array}{cc}
A & 0_{r, r} \\
0_{r, r} & A^{-t}
\end{array}\right) \text { for } A \in G L(r)\right\} \cong G L(r) .
$$

For the last claim in the case $r=1$ it is enough to note that $S O(2) \cap S p(2)=S O(2)$. In fact every $2 \times 2$ matrix with determinant one is symplectic.

Proposition 4.3. Let $O_{2 r} \subset X_{2 r}$ be the orbit of the identity. Then $\operatorname{Pic}\left(O_{2 r}\right) \cong \mathbb{Z} / 2 \mathbb{Z}$.

Proof. The group $G=S p(2 r)$ is semi-simple and simply connected. If $H \subset G$ is the stabilizer of the identity then ADHL15, Theorem 4.5.1.2] yields that $\operatorname{Pic}(G / H) \cong \mathbb{X}(H)$, where $\mathbb{X}(H)$ is the group of characters of $H$. We have that

$$
H=\left\{M \in S p(2 r), M M^{t}=\lambda_{M} I_{2 r, 2 r}, \text { for some } \lambda_{M} \in K^{*}\right\} .
$$

Then, for a general element $M \in H$ we have

$$
\left\{\begin{array}{l}
M M^{t}=\lambda_{M} I_{2 r, 2 r} ; \quad \Rightarrow \lambda_{M} M^{-1} \Omega M=\Omega \Rightarrow \lambda_{M} \Omega M=M \Omega . \\
M^{t} \Omega M=\Omega ;
\end{array}\right.
$$

Let $v$ be an eigenvector of $\Omega$ with eigenvalue $\mu$. Then

$$
\lambda_{M} \Omega M v=M \Omega v=M \mu v=\mu M v .
$$


Setting $y=M v$ we have $\Omega y=\left(\lambda_{M}^{-1} \mu\right) y$ and so $y$ is an eigenvector of $\Omega$ with eigenvalue $\lambda_{M}^{-1} \mu$. The characteristic polynomial of $\Omega$ is $P_{\Omega}(\lambda)=(\lambda-\xi)^{r}(\lambda+\xi)^{r}$ where $\xi^{2}=-1$. Therefore the only eigenvalues of $\Omega$ are $\xi$ and $-\xi$. So

$$
\left\{\begin{array}{l}
\mu= \pm \xi ; \\
\lambda_{M}^{-1} \mu= \pm \xi ;
\end{array} \quad \Rightarrow \lambda_{M}^{-1}= \pm 1 \Rightarrow \lambda_{M}= \pm 1\right.
$$

and there is a morphism of groups

$$
\begin{aligned}
\varphi: H & \longrightarrow \mathbb{Z} / 2 \mathbb{Z} \\
M & \longmapsto \lambda_{M}
\end{aligned}
$$

The morphism $\varphi$ is surjective. Indeed we have $\varphi\left(I_{2 r, 2 r}\right)=1$, and if $S=\left(\begin{array}{cc}0_{r, r} & \xi I_{r, r} \\ \xi I_{r, r} & 0_{r, r}\end{array}\right)$ then $S^{t} \Omega S=\Omega, S S^{t}=$ $-I_{2 r, 2 r}, S \in H$ and $\varphi(S)=-1$. This yields an exact sequence

$$
1 \rightarrow \bar{H} \rightarrow H \rightarrow \mathbb{Z} / 2 \mathbb{Z} \rightarrow 1
$$

where $\bar{H}=\left\{M \in S p(2 r), M M^{t}=I_{2 r, 2 r}\right\}$, and we can write $H=\bar{H} \cup S \bar{H}$.

As in Lemma 4.2, we consider the bilinear form $J=\left(\begin{array}{ll}0_{r, r} & I_{r, r} \\ I_{r, r} & 0_{r, r}\end{array}\right)$, which is congruent to the bilinear form $I_{2 r, 2 r}$ via the matrix $N=\left(\begin{array}{cc}I_{r, r} & \xi I_{r, r} \\ \frac{1}{2} I_{r, r} & -\frac{\xi}{2} I_{r, r}\end{array}\right)$, where $\xi^{2}=-1$. Set $\bar{H}_{J}=\left\{M \in S p(2 r), M J M^{t}=J\right\}$ and $H_{J}=\{M \in$ $S p(2 r), M J M^{t}=\lambda_{M} J$, for some $\left.\lambda_{M} \in K^{*}\right\}$. There is an isomorphism

$$
\begin{aligned}
\alpha: H & \longrightarrow H_{J} \\
M & \longmapsto N M N^{-1}
\end{aligned}
$$

such that $\alpha(\bar{H})=\bar{H}_{J}, \tilde{S}:=\alpha(S)=\left(\begin{array}{cc}0 & -2 I_{r, r} \\ \frac{1}{2} I_{r, r} & 0\end{array}\right)$ and $H_{J}=\bar{H}_{J} \cup \tilde{S} \bar{H}_{J}$. Take $B \in H_{J}$ and consider $\alpha^{-1}(B) \in H$. By the first part of the proof there is a morphism of groups $H_{J} \rightarrow \mathbb{Z} / 2 \mathbb{Z}$ mapping $B$ to $\lambda_{\alpha^{-1}(B)}$, and fitting in the following exact sequence

$$
1 \rightarrow \bar{H}_{J} \rightarrow H_{J} \rightarrow \mathbb{Z} / 2 \mathbb{Z} \rightarrow 1
$$

Since $H_{J} / \bar{H}_{J}$ is abelian the commutator $\left[H_{J}, H_{J}\right]$ of $H_{J}$ is contained in $\bar{H}_{J}$. By the proof of Lemma 4.2 we have that an element $h \in \bar{H}_{J}$ is of the form $h=\left(\begin{array}{cc}A & 0_{r, r} \\ 0_{r, r} & A^{-t}\end{array}\right)$ for $A \in G L(r)$. Then $h^{-1}=\left(\begin{array}{cc}A^{-1} & 0_{r, r} \\ 0_{r, r} & A^{t}\end{array}\right)$ for $A \in G L(r)$. Furthermore $\tilde{S}^{-1}=\left(\begin{array}{cc}0_{r, r} & 2 I_{r, r} \\ -\frac{1}{2} I_{r, r} & 0_{r, r}\end{array}\right)$. Therefore

$$
[\tilde{S}, h]=\tilde{S} h \tilde{S}^{-1} h^{-1}=\left(\begin{array}{cc}
0_{r, r} & -2 I_{r, r} \\
\frac{1}{2} I_{r, r} & 0_{r, r}
\end{array}\right)\left(\begin{array}{cc}
A & 0_{r, r} \\
0_{r, r} & A^{-t}
\end{array}\right)\left(\begin{array}{cc}
0_{r, r} & 2 I_{r, r} \\
-\frac{1}{2} I_{r, r} & 0_{r, r}
\end{array}\right)\left(\begin{array}{cc}
A^{-1} & 0_{r, r} \\
0_{r, r} & A^{t}
\end{array}\right)=\left(\begin{array}{cc}
A^{-t} A^{-1} & 0_{r, r} \\
0_{r, r} & A A^{t}
\end{array}\right) .
$$

Setting $B=A^{-t} A^{-1}$, we have $B^{-t}=\left(A^{-t} A^{-1}\right)^{-t}=A A^{t}$ with $B \in G L(r)$ symmetric. So $\left[H_{J}, H_{J}\right]$ is the subgroup of $\bar{H}_{J} \cong G L(r)$ generated by symmetric matrices and since by [Bos86, Theorem 1] all $r \times r$ matrices can be written as product of symmetric matrices we get $\left[H_{J}, H_{J}\right]=\bar{H}_{J}$.

Then, $H /[H, H] \cong H_{J} /\left[H_{J}, H_{J}\right] \cong H_{J} / \bar{H}_{J} \cong H / \bar{H}$ and by the exact sequence (4.4) we have $H / \bar{H} \cong \mathbb{Z} / 2 \mathbb{Z}$. Finally, by [Bur65, Lemma 22.2] $\mathbb{X}(H) \cong \mathbb{X}(H /[H, H])$, and hence $\operatorname{Pic}(G / H) \cong \mathbb{X}(H) \cong \mathbb{Z} / 2 \mathbb{Z}$.

Now, we are ready to compute the Picard rank and the colors of the wonderful variety $\mathcal{S}_{2 r}$.

Proposition 4.5. The Picard rank of $\mathcal{S}_{2 r}$ is $\rho\left(\mathcal{S}_{2 r}\right)=r$.

Proof. As before set $G=S p(2 r)$ and let $H$ be the stabilizer of the identity. By Theorem 3.19 the variety $\mathcal{S}_{2 r}$ is wonderful with boundary divisors $E_{1}, \ldots, E_{r-1}, S_{r}^{(r-1)}\left(\mathcal{V}_{2}^{2 r-1}\right)$. By [Bri07, Proposition 2.2.1] there is an exact sequence

$$
0 \rightarrow \mathbb{Z}^{r} \rightarrow \operatorname{Pic}\left(\mathcal{S}_{2 r}\right) \rightarrow \operatorname{Pic}(G / H) \rightarrow 0
$$

Hence, Proposition 4.3 yields that the Picard rank of $\mathcal{S}_{2 r}$ is $r$.

For $i=1, \ldots, r$ we define the divisors $D_{i}$ as the strict transforms in $\mathcal{S}_{2 r}$ of the divisor given by the intersection of

$$
\operatorname{det}\left(\begin{array}{ccc}
z_{0,0} & \cdots & z_{0, i-1} \\
\vdots & \ddots & \vdots \\
z_{0, i-1} & \cdots & z_{i-1, i-1}
\end{array}\right)=0
$$


with $X_{2 r}$.

Proposition 4.6. The set of boundary divisors of $\mathcal{S}_{2 r}$ is $\left\{E_{1}, \ldots, E_{r-1}, S_{r}^{r-1}\left(\mathcal{V}_{2}^{2 r-1}\right)\right\}$ while the set of colors of $\mathcal{S}_{2 r}$ is $\left\{D_{1}, \ldots, D_{r}\right\}$.

Proof. The claim on the set of boundary divisors follows from Theorem 3.19. We compute the colors. We first prove that $D_{r} \subseteq \mathcal{S}_{2 r}$ is stabilized by the Borel subgroup. Consider a matrix $Z=\left(\begin{array}{cc}Z_{0,0} & Z_{0,1} \\ Z_{0,1} & Z_{1,1}\end{array}\right)$ where the $Z_{i, j}$ are $r \times r$ matrices. Let $M=\left(\begin{array}{cc}A & 0_{r, r} \\ B & A^{-t}\end{array}\right) \in \mathscr{B}$, then

$$
\bar{Z}=M \cdot Z \cdot M^{t}=\left(\begin{array}{cc}
A Z_{0,0} A^{t} & A Z_{0,0} B^{t}+A Z_{0,1} A^{-1} \\
B Z_{0,0} A^{t}+A^{-t} Z_{0,1} A^{t} & B Z_{0,0} B^{t}+A^{-t} Z_{0,1} B^{t}+B Z_{0,1} A^{-1}+A^{-t} Z_{1,1} A^{-1}
\end{array}\right)
$$

and $\operatorname{det}\left(\bar{Z}_{0,0}\right)=\operatorname{det}\left(A Z_{0,0} A^{t}\right)=\operatorname{det}(A)^{2} \operatorname{det}\left(Z_{0,0}\right)$ where $\operatorname{det}(A) \neq 0$ since $A \in G L(r)$. Therefore, $D_{r}$ is stabilized by the Borel subgroup.

We focus now on the block $\bar{Z}_{0,0}$ of the matrix $\bar{Z}$. We divide the matrices $A$ and $Z_{0,0}$ respectively in blocks $A_{j, k}$, $W_{j, k}$ of matrices $j \times k$ as follows $A=\left(\begin{array}{cc}A_{i, i} & A_{i, r-i} \\ A_{r-i, i} & A_{r-i, r-i}\end{array}\right)$ and $Z_{0,0}=\left(\begin{array}{cc}W_{i, i} & W_{i, r-i} \\ W_{r-i, i} & W_{r-i, r-i}\end{array}\right)$. Recall that by Remark 3.4 the matrix $A$ is lower triangular. We have $\bar{Z}_{0,0}=\left(\begin{array}{cc}\bar{W}_{i, i} & \bar{W}_{i, r-i} \\ \bar{W}_{r-i, i} & \bar{W}_{r-i, r-i}\end{array}\right)$ with $\bar{W}_{i, i}=A_{i, i} W_{i, i} A_{i, i}^{t}$. The divisor $D_{i}$ is defined by $\operatorname{det}\left(W_{i, i}\right)=0$ and $\operatorname{since} \operatorname{det}(A)=\operatorname{det}\left(A_{i, i}\right) \operatorname{det}\left(A_{r-i, r-i}\right) \neq 0$ we get that $D_{i}$ is stabilized by $\mathscr{B}$ for $i=1, \ldots r$.

As noticed in [ADHL15, Remark 4.5.5.3], if $\left(X, \mathscr{G}, \mathscr{B}, x_{0}\right)$ is a spherical wonderful variety with colors $D_{1}, \ldots, D_{s}$ the big cell $X \backslash\left(D_{1} \cup \cdots \cup D_{s}\right)$ is an affine space. Therefore, it admits only constant invertible global functions and $\operatorname{Pic}(X)$ is generated by $D_{1}, \ldots, D_{s}$.

Therefore, in order to conclude that we found all the colors of $\mathcal{S}_{2 r}$ it is enough to recall that by Proposition 4.5 $\mathcal{S}_{2 r}$ has Picard rank $r$.

In the following we will denote by $H$ the pull-back in $\mathcal{S}_{2 r}$ of the hyperplane section of $X_{2 r}$. By Proposition 4.5 $H, E_{1} \ldots, E_{r-1}$ generate $\operatorname{Pic}\left(\mathcal{S}_{2 r}\right)$.

Proposition 4.7. The extremal rays of $\operatorname{Eff}\left(\mathcal{S}_{2 r}\right)$ are generated by $E_{1}, \ldots, E_{r-1}, S_{r}^{r-1}\left(\mathcal{V}_{2}^{2 r-1}\right)$ and the extremal rays of $\operatorname{Nef}\left(\mathcal{S}_{2 r}\right)$ are generated by $D_{1}, \ldots, D_{r}$.

Proof. By ADHL15, Proposition 4.5.4.4] and Proposition 4.6 Eff $\left(\mathcal{S}_{2 r}\right)$ is generated by $E_{1}, \ldots, E_{r-1}, S_{r}^{r-1}\left(\mathcal{V}_{2}^{2 r-1}\right)$ and $D_{1}, \ldots, D_{r}$.

Note that by Constructions 2.5 and 3.11 there as an inclusion $i: \mathcal{S}_{2 r} \rightarrow \mathcal{Q}(2 r-1)_{r-1}$ inducing an isomorphism of the Picard groups. By [Hue15, Section 2] the linear system on $\mathcal{Q}(2 r-1)_{r-1}$ that restricts to the linear system of $D_{i}$ on $\mathcal{S}_{2 r}$ induces a birational morphism $\mathcal{Q}(2 r-1)_{r-1} \rightarrow W_{i}$ whose exceptional locus is contained in the union of the exceptional divisors in Construction 2.5. Therefore, $D_{i}$ induces a birational morphism $\mathcal{S}_{2 r} \rightarrow Z_{i}$ and hence $D_{i}$ lies in the interior of the effective cone of $\mathcal{S}_{2 r}$ for any $i=1, \ldots, r$. This proves that the effective cone of $\mathcal{S}_{2 r}$ is generated by $E_{1}, \ldots, E_{r-1}, S_{r}^{r-1}\left(\mathcal{V}_{2}^{2 r-1}\right)$. Finally, by [Bri89, Section 2.6] $D_{1}, \ldots, D_{r}$ generate the extremal rays of the nef cone.

In order to study the birational geometry of $\mathcal{S}_{2 r}$ we will need the following result.

Proposition 4.8. Let $H_{i}^{r}$ be the divisor in $X_{2 r} \subset \mathbb{P}^{N}$ cut out by the determinant of the $i \times i$ top left submatrix of the matrix $Z$ in (2.1). The tangent cone of $H_{i}^{r}$ at a point of $S_{k}\left(\mathcal{V}_{2}^{2 r-1}\right) \backslash S_{k-1}\left(\mathcal{V}_{2}^{2 r-1}\right)$ for $i=2, \ldots, r$ and $k<i$ is a cone with vertex of dimension $k(2 r+1-k)$ over $H_{i-k}^{r-k}$.

Proof. It is enough to note that the tangent cone of $H_{i}^{r}$ at the point $p_{k}=\left(p_{i, j}\right)_{i, j=0, \ldots, 2 r-1}$, where $p_{i, i}=1$ for $i=0, \ldots, k-1$ and $p_{i, j}=0$ otherwise, is cut out by

$$
\operatorname{det}\left(\begin{array}{cccc}
z_{k, k} & z_{k, k+1} & \ldots & z_{k, i-1} \\
z_{k, k+1} & z_{k+1, k+1} & \ldots & z_{k+1, i-1} \\
\vdots & \vdots & \ddots & \vdots \\
z_{k, i-1} & z_{k+1, i-1} & \ldots & z_{i-1, i-1}
\end{array}\right)=0
$$

and by the equations for the tangent cone of $X_{2 r}$ in the proof of Proposition 3.18 


\section{Birational geometry of $\mathcal{S}_{2 r}$}

The stable base locus of an effective $\mathbb{Q}$-divisor on a normal $\mathbb{Q}$-factorial projective variety $X$ has been defined in (4.1). Since stable base loci do not behave well with respect to numerical equivalence [Laz04, Example 10.3.3], we will assume that $h^{1}\left(X, \mathcal{O}_{X}\right)=0$ so that linear and numerical equivalence of $\mathbb{Q}$-divisors coincide.

Then numerically equivalent $\mathbb{Q}$-divisors on $X$ have the same stable base locus, and the pseudo-effective cone $\overline{\mathrm{Eff}}(X)$ of $X$ can be decomposed into chambers depending on the stable base locus of the corresponding linear series. The resulting decomposition is called stable base locus decomposition.

Remark 5.1. Recall that two divisors $D_{1}, D_{2}$ are said to be Mori equivalent if $\mathbf{B}\left(D_{1}\right)=\mathbf{B}\left(D_{2}\right)$ and the following diagram of rational maps is commutative

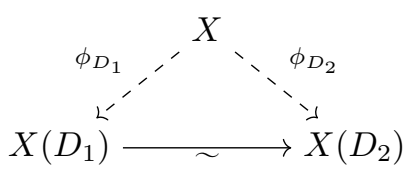

where the horizontal arrow is an isomorphism. Therefore, the Mori chamber decomposition is a, possibly trivial, refinement of the stable base locus decomposition.

Let $X$ be a normal $\mathbb{Q}$-factorial variety with free and finitely generated divisor class group $\mathrm{Cl}(X)$. Fix a subgroup $G$ of the group of Weil divisors on $X$ such that the canonical map $G \rightarrow \mathrm{Cl}(X)$, mapping a divisor $D \in G$ to its class $[D]$, is an isomorphism. The Cox ring of $X$ is defined as

$$
\operatorname{Cox}(X)=\bigoplus_{[D] \in \operatorname{Cl}(X)} H^{0}\left(X, \mathcal{O}_{X}(D)\right)
$$

where $D \in G$ represents $[D] \in \mathrm{Cl}(X)$, and the multiplication in $\operatorname{Cox}(X)$ is defined by the standard multiplication of homogeneous sections in the field of rational functions on $X$. If $\operatorname{Cox}(X)$ is finitely generated as an algebra over the base field, then $X$ is said to be a Mori dream space. A perhaps more enlightening definition, especially for the relation with the minimal model program, is the following.

Definition 5.2. A normal projective $\mathbb{Q}$-factorial variety $X$ is called a Mori dream space if the following conditions hold:

- $\operatorname{Pic}(X)$ is finitely generated, or equivalently $h^{1}\left(X, \mathcal{O}_{X}\right)=0$,

- Nef $(X)$ is generated by the classes of finitely many semi-ample divisors,

- there is a finite collection of small $\mathbb{Q}$-factorial modifications $f_{i}: X \rightarrow X_{i}$, such that each $X_{i}$ satisfies the second condition above, and $\operatorname{Mov}(X)=\bigcup_{i} f_{i}^{*}\left(\operatorname{Nef}\left(X_{i}\right)\right)$.

The collection of all faces of all cones $f_{i}^{*}\left(\operatorname{Nef}\left(X_{i}\right)\right)$ above forms a fan which is supported on $\operatorname{Mov}(X)$. If two maximal cones of this fan, say $f_{i}^{*}\left(\operatorname{Nef}\left(X_{i}\right)\right)$ and $f_{j}^{*}\left(\operatorname{Nef}\left(X_{j}\right)\right)$, meet along a facet, then there exist a normal projective variety $Y$, a small modification $\varphi: X_{i} \rightarrow X_{j}$, and $h_{i}: X_{i} \rightarrow Y, h_{j}: X_{j} \rightarrow Y$ small birational morphisms of relative Picard number one such that $h_{j} \circ \varphi=h_{i}$. The fan structure on $\operatorname{Mov}(X)$ can be extended to a fan supported on $\operatorname{Eff}(X)$ as follows.

Definition 5.3. Let $X$ be a Mori dream space. We describe a fan structure on the effective cone Eff $(X)$, called the Mori chamber decomposition. We refer to [HK00, Proposition 1.11] and [Oka16, Section 2.2] for details. There are finitely many birational contractions from $X$ to Mori dream spaces, denoted by $g_{i}: X \rightarrow Y_{i}$. The set $\operatorname{Exc}\left(g_{i}\right)$ of exceptional prime divisors of $g_{i}$ has cardinality $\rho\left(X / Y_{i}\right)=\rho(X)-\rho\left(Y_{i}\right)$. The maximal cones $\mathcal{C}$ of the Mori chamber decomposition of $\operatorname{Eff}(X)$ are of the form: $\mathcal{C}_{i}=\left\langle g_{i}^{*}\left(\operatorname{Nef}\left(Y_{i}\right)\right), \operatorname{Exc}\left(g_{i}\right)\right\rangle$. We call $\mathcal{C}_{i}$ or its interior $\mathcal{C}_{i}^{\circ}$ a maximal chamber of $\operatorname{Eff}(X)$.

If $X$ is a Mori dream space, satisfying then the condition $h^{1}\left(X, \mathcal{O}_{X}\right)=0$, determining the stable base locus decomposition of $\operatorname{Eff}(X)$ is a first step in order to compute its Mori chamber decomposition.

Remark 5.4. By the work of $M$. Brion [Bri93] we have that $\mathbb{Q}$-factorial spherical varieties are Mori dream spaces. An alternative proof of this result can be found in [Per14, Section 4]. In particular, by Theorem 3.19 the wonderful compactification $\mathcal{S}_{2 r}$ is a Mori dream space.

Remark 5.5. Recall that by [HK00, Proposition 2.11] given a Mori Dream Space $X$ there is an embedding $i: X \rightarrow \mathcal{T}_{X}$ into a simplicial projective toric variety $\mathcal{T}_{X}$ such that $i^{*}: \operatorname{Pic}\left(\mathcal{T}_{X}\right) \rightarrow \operatorname{Pic}(X)$ is an isomorphism inducing an isomorphism $\operatorname{Eff}\left(\mathcal{T}_{X}\right) \rightarrow \operatorname{Eff}(X)$. Furthermore, the Mori chamber decomposition of $\operatorname{Eff}\left(\mathcal{T}_{X}\right)$ is a refinement of the Mori chamber decomposition of $\operatorname{Eff}(X)$. Indeed, if $\operatorname{Cox}(X) \cong \frac{K\left[T_{1}, \ldots, T_{s}\right]}{I}$ where the $T_{i}$ are homogeneous generators with non-trivial effective $\operatorname{Pic}(X)$-degrees then $\operatorname{Cox}\left(\mathcal{T}_{X}\right) \cong K\left[T_{1}, \ldots, T_{s}\right]$. 
Since the variety $\mathcal{T}_{X}$ is toric, the Mori chamber decomposition of $\operatorname{Eff}\left(\mathcal{T}_{X}\right)$ can be computed by means of the Gelfand-Kapranov-Zelevinsky, GKZ for short, decomposition [ADHL15, Section 2.2.2]. Let us consider the family $\mathcal{W}$ of vectors in $\operatorname{Pic}\left(\mathcal{T}_{X}\right)$ given by the generators of $\operatorname{Cox}\left(\mathcal{T}_{X}\right)$, and let $\Omega(\mathcal{W})$ be the set of all convex polyhedral cones generated by some of the vectors in $\mathcal{W}$. By [ADHL15, Construction 2.2.2.1] the GKZ chambers of $\operatorname{Eff}\left(\mathcal{T}_{X}\right)$ are given by the intersections of all the cones in $\Omega(\mathcal{W})$ containing a fixed divisor in $\operatorname{Eff}\left(\mathcal{T}_{X}\right)$.

Remark 5.6. Let $\left(X, \mathscr{G}, \mathscr{B}, x_{0}\right)$ be a projective spherical variety. Consider a divisor $D$ on $X$, and let $f_{D}$ be the, unique up to constants, section of $\mathcal{O}_{X}(D)$ associated to $D$. We will denote by $\operatorname{lin}_{K}(\mathscr{G} \cdot D) \subseteq \operatorname{Cox}(X)$ the finite-dimensional vector subspace of $\operatorname{Cox}(X)$ spanned by the orbit of $f_{D}$ under the action of $\mathscr{G}$ that is the smallest linear subspace of $\operatorname{Cox}(X)$ containing the $\mathscr{G}$-orbit of $f_{D}$.

By [ADHL15, Theorem 4.5.4.6] if $\mathscr{G}$ is a semi-simple and simply connected algebraic group and $\left(X, \mathscr{G}, \mathscr{B}, x_{0}\right)$ is a spherical variety with boundary divisors $E_{1}, \ldots, E_{r}$ and colors $D_{1}, \ldots, D_{s}$ then $\operatorname{Cox}(X)$ is generated as a $K$-algebra by the canonical sections of the $E_{i}$ 's and the finite dimensional vector $\operatorname{subspaces} \operatorname{lin}_{K}\left(\mathscr{G} \cdot D_{i}\right) \subseteq \operatorname{Cox}(X)$ for $1 \leqslant i \leqslant s$.

Definition 5.7. Let $X$ be a normal projective $\mathbb{Q}$-factorial variety. We say that $X$ is weak Fano if $-K_{X}$ is nef and big.

By [BCHM10, Corollary 1.3.2] a weak Fano variety is a Mori dream space.

Remark 5.8. Let $Y$ be a smooth and irreducible subvariety of a smooth variety $X$, and let $f: B l_{Y} X \rightarrow X$ be the blow-up of $X$ along $Y$ with exceptional divisor $E$. Then for any divisor $D \in \operatorname{Pic}(X)$ in $\operatorname{Pic}\left(B l_{Y} X\right)$ we have

$$
\widetilde{D} \sim f^{*} D-\operatorname{mult}_{Y}(D) E
$$

where $\widetilde{D} \subset B l_{Y} X$ is the strict transform of $D$, and mult $\operatorname{li}_{Y}(D)$ is the multiplicity of $D$ at a general point of $Y$.

Corollary 5.9. The Cox ring of $\mathcal{S}_{2 r}$ is generated by the sections of $D_{1}, \ldots D_{r}, E_{1}, \ldots, E_{r-1}, S_{r}^{(r-1)}\left(\mathcal{V}_{r}^{2 r-1}\right)$.

Proof. This follows from Proposition 4.6 and Remark 5.6 .

Our aim is to study the Mori chamber decomposition of the wonderful compactification $\mathcal{S}_{2 r}$. Since $\mathcal{S}_{2} \cong \mathbb{P}^{2}$ the first interesting case is for $r=2$.

Proposition 5.10. For the variety $\mathcal{S}_{4}$ we have that $\operatorname{Pic}\left(\mathcal{S}_{4}\right)$ is generated by $D_{1}, E_{1}$. Furthermore, $D_{1} \sim H$, $D_{2} \sim 2 H-E_{1}, S_{2}^{(1)}\left(\mathcal{V}_{2}^{3}\right) \sim 2 H-2 E_{1}$, and $\operatorname{Cox}\left(\mathcal{S}_{4}\right)$ is generated by the sections of $D_{1}, D_{2}, E_{1}, S_{2}^{(1)}\left(\mathcal{V}_{2}^{3}\right)$. The Mori chamber decomposition of $\mathrm{Eff}\left(\mathcal{S}_{4}\right)$ has three chambers as displayed in the following picture:

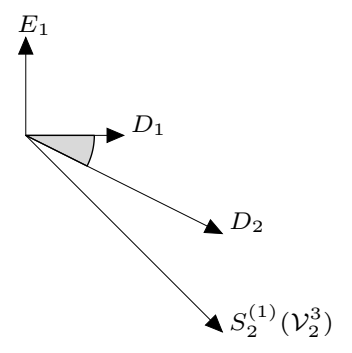

and the movable cone coincides with the nef cone generated by $D_{1}$ and $D_{2}$.

Proof. Since $\mathcal{S}_{4}$ is the blow-up of a smooth variety along a smooth subvariety the relations $D_{2} \sim 2 H-E_{1}$, $S_{2}^{(1)}\left(\mathcal{V}_{2}^{3}\right) \sim 2 H-2 E_{1}$ follow from Propositions 3.18, 3.21, 4.8 and Remark 5.8,

The statement on the generators of the Cox ring follows from Corollary 5.9. Furthermore, by Remarks 5.5 and [5.6] the Mori chamber decomposition of $\operatorname{Eff}\left(\mathcal{S}_{4}\right)$ is a, possibly trivial, coarsening of the decomposition in the statement. On the other hand, by Proposition 4.7 we know that $H$ and $2 H-E_{1}$ generate $\operatorname{Nef}\left(\mathcal{S}_{4}\right)$ while $E_{1}$ and $2 H-2 E_{1}$ generate $\operatorname{Eff}\left(\mathcal{S}_{4}\right)$. So no ray can be removed and the above decomposition coincides with the Mori chamber decomposition of $\operatorname{Eff}\left(\mathcal{S}_{4}\right)$.

Next, we consider the case $r=3$.

Lemma 5.11. For the variety $\mathcal{S}_{6}$ the Picard group $\operatorname{Pic}\left(\mathcal{S}_{6}\right)$ is generated by $H, E_{1}, E_{2}$, and we have the following relations: $D_{1} \sim H, D_{2} \sim 2 H-E_{1}, D_{3} \sim 3 H-2 E_{1}-E_{2}$ and $S_{3}^{2}\left(\mathcal{V}_{2}^{5}\right) \sim 2 H-2 E_{1}-2 E_{2}$. 
Proof. Recall, that the first blow-up $f_{1}: X_{6}^{(1)} \rightarrow X_{6}$ in Construction 3.11 is the blow-up of $X_{6}$ along the Veronese variety $\mathcal{V}_{2}^{5}$ which by Proposition 3.18 is the singular locus of $X_{6}$. Hence, in this case we can not use Remark 5.8 to compute the discrepancies of the relevant divisors with respect to $E_{1}$. In order to do this we consider the line $L=\left\{z_{1,1}-z_{0,1}=z_{1,1}-z_{2,2}=z_{0,2}=z_{0,3}=z_{0,4}=z_{0,5}=z_{1,2}=z_{1,3}=z_{1,4}=z_{1,5}=z_{2,3}=z_{2,4}=z_{2,5}=z_{3,3}=\right.$ $\left.z_{3,4}=z_{3,5}=z_{4,4}=z_{4,5}=z_{5,5}=0\right\}$ and let $\tilde{L}$ be its strict transform in $X_{6}^{(1)}$. Slightly abusing the notation we will denote by $D_{i}$ also the strict transform in $X_{6}^{(1)}$ of the divisor $H_{i}^{3}$ in Proposition 4.8 for $i=1,2,3$ and by $H$ the pull-back of the hyperplane section to $X_{6}^{(1)}$. Clearly, $D_{1} \sim H$.

Now, let us write $D_{2} \sim 2 H-a E_{1}$. Note that the line $L$ intersects $\mathcal{V}_{2}^{5}$ just at the point $p=[1: 0 \cdots: 0]$, and by Remark 3.10 and Proposition $3.18 L \subset X_{6}$. By Proposition 3.18 the tangent cone of $X_{6}$ at $p$ is a cone over $X_{4} \cong \mathbb{G}(1,4)$ with 5 -dimensional vertex and $\widetilde{L}$ intersects $E_{1}$ just at the point $q=[1: 0: 0: 0: 1: 0: \cdots: 0]$ of $X_{4}$. Hence $\widetilde{L} \cdot E_{1}=1$. The divisor $H_{2}^{3}$ intersects $L$ in $p$ and in another point not lying on $\mathcal{V}_{2}^{5}$. Moreover, by Proposition 4.8 the tangent cone of $H_{2}^{3}$ at $p$ is a hyperplane section of $X_{4}$ not passing through $q$. Then $\widetilde{L} \cdot D_{2}=1$. By the projection formula we have

$$
1=\widetilde{L} \cdot D_{2}=2 \widetilde{L} \cdot H-a \widetilde{L} \cdot E_{1}=2 L \cdot H_{1}^{3}-a=2-a
$$

and hence $a=1$. So we may write $D_{2} \sim 2 H-E_{1}$.

Now, write $D_{3} \sim 3 H-b E_{1}$. The divisor $H_{3}^{3}$ intersects $L$ in $p$ with multiplicity two and in another point not lying on $\mathcal{V}_{2}^{5}$. By Proposition 4.8 the tangent cone of $H_{3}^{3}$ at $p$ is a quadratic section of $X_{4}$ not passing through $q$. Hence

$$
1=\widetilde{L} \cdot D_{3}=3 \widetilde{L} \cdot H-a \widetilde{L} \cdot E_{1}=3 L \cdot H_{1}^{3}-a=3-a
$$

and $a=2$. Then $D_{3} \sim 3 H-2 E_{1}$.

We will denote by $S_{3}$ the strict transform of $S_{3}\left(\mathcal{V}_{2}^{5}\right)$ in $X_{6}^{(1)}$. Let $R \subset X_{4} \cong \mathbb{G}(1,4)$ be a general line. Note that $R$ is contracted by the blow-down morphism and hence

$$
1=R \cdot D_{2}=2 R \cdot H-R \cdot E_{1}=-R \cdot E_{1}
$$

yields $R \cdot E_{1}=-1$. By Proposition 3.21 we may write $S_{3} \sim 2 H-c E_{1}$ and since by Proposition 3.18 the tangent cone of $S_{3}\left(\mathcal{V}_{2}^{5}\right)$ at a point of $\mathcal{V}_{2}^{5}$ is a quadratic section of $X_{4}$ we have $R \cdot S_{3}=2$. This yields

$$
2=R \cdot S_{3}=2 R \cdot H-c R \cdot E_{1}=-c R \cdot E_{1}=c
$$

and $S_{3} \sim 2 H-2 E_{1}$.

Now, by Proposition 3.18 the morphism $f_{2}: \mathcal{S}_{6} \rightarrow X_{6}^{(1)}$ in Construction 3.11 is the blow-up of a smooth variety along a smooth subvariety. So we can apply Remark 5.8 in order to compute the discrepancies of the divisors with respect to $E_{2}$. Finally, again by Proposition 3.18 we get the claim.

Theorem 5.12. The Cox ring of $\mathcal{S}_{6}$ is generated by the sections of $D_{1}, D_{2}, D_{3}, E_{1}, E_{2}, S_{3}^{(2)}\left(\mathcal{V}_{2}^{5}\right)$. The Mori chamber decomposition of the effective cone of $\mathcal{S}_{6}$ has nine chambers as displayed in the following 2-dimensional section of $\operatorname{Eff}\left(\mathcal{S}_{6}\right)$ :

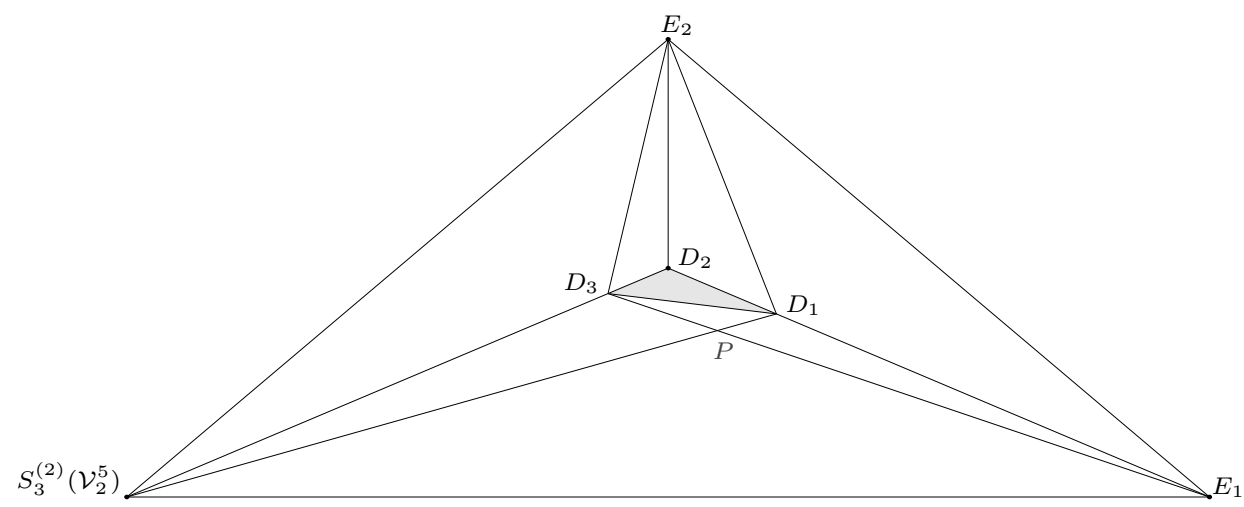

where $P \sim 3 H-E_{1}-E_{2}$ and $\operatorname{Mov}\left(\mathcal{S}_{6}\right)$ is generated by $D_{1}, D_{2}, D_{2}$ and $P$.

Proof. The computation of the movable cone follows from ADHL15, Proposition 3.3.2.3], Proposition 4.6 and Remark 5.6, and the statement on the generators of $\operatorname{Cox}\left(\mathcal{S}_{6}\right)$ follows from Corollary 5.9.

Furthermore, by Lemma 5.11, Proposition 4.6 and Remarks 5.5, 5.6 the Mori chamber decomposition of Eff $\left(\mathcal{S}_{6}\right)$ is a, possibly trivial, coarsening of the decomposition in the statement. 
Note that the stable base loci of a divisor in the interior of chamber delimited by $S_{3}^{(2)}\left(\mathcal{V}_{2}^{5}\right), P, E_{1} ; S_{3}^{2}\left(\mathcal{V}_{2}^{5}\right), P, D_{3}$; $S_{3}^{(2)}\left(\mathcal{V}_{2}^{5}\right), D_{3}, E_{2} ; D_{2}, D_{3}, D_{1}, E_{2} ; E_{1}, D_{1}, E_{2} ; P, D_{1}, E_{1}$ are respectively given by $S_{3}^{(2)}\left(\mathcal{V}_{2}^{5}\right) \cup E_{1} ; S_{3}^{2}\left(\mathcal{V}_{2}^{5}\right) ; E_{2} \cup$ $S_{3}^{(2)}\left(\mathcal{V}_{2}^{5}\right) ; E_{2} ; E_{1} \cup E_{2} ; E_{1}$. Furthermore, since Mori chambers are convex the stable base locus chamber delimited by $D_{2}, D_{3}, D_{1}, E_{2}$ must be divided in two Mori chambers by the wall joining $D_{2}$ and $E_{2}$. Hence the decomposition in the statement gives the Mori chamber decomposition of $\operatorname{Eff}\left(\mathcal{S}_{6}\right)$ outside of the movable cone.

Finally, note that the only modifications we could perform inside the movable cone are removing the wall joining $D_{1}$ and $D_{3}$ and adding a wall joining $D_{2}$ and $P$. However, both these modifications are not allowed since by Proposition 4.7 the chamber delimited by $D_{1}, D_{2}, D_{3}$ is the nef cone of $\mathcal{S}_{6}$.

\section{Moduli spaces of COnics in Lagrangian Grassmannians}

An $n$-pointed rational pre-stable curve $\left(C,\left(x_{1}, \ldots, x_{n}\right)\right)$ is a projective, connected, reduced rational curve with at most nodal singularities of arithmetic genus zero, with $n$ distinct and smooth marked points $x_{1}, \ldots, x_{n} \in C$. We will refer to the marked and the singular points of $C$ as special points.

Let $X$ be a homogeneous variety. A map $\left(C,\left(x_{1}, \ldots, x_{n}\right), \alpha\right)$, where $\alpha: C \rightarrow X$ is a morphism from an $n$-pointed rational pre-stable curve to $X$, is stable if any component $E \cong \mathbb{P}^{1}$ of $C$ contracted by $\alpha$ contains at least three special points.

Now, let us fix a class $\beta \in H_{2}(X, \mathbb{Z})$. By [FP97, Theorem 2] there exists a smooth, proper, and separated Deligne-Mumford stack $\overline{\mathcal{M}}_{0, n}(X, \beta)$ parametrizing isomorphism classes of stable maps $\left[C,\left(x_{1}, \ldots, x_{n}\right), \alpha\right]$ such that $\alpha_{*}[C]=\beta$. Furthermore, by [KP01, Corollary 1] the coarse moduli space $\bar{M}_{0, n}(X, \beta)$ associated to the stack $\overline{\mathcal{M}}_{0, n}(X, \beta)$ is a normal, irreducible, projective variety with at most finite quotient singularities of dimension

$$
\operatorname{dim}\left(\bar{M}_{0, n}(X, \beta)\right)=\operatorname{dim}(X)+\beta \cdot c_{1}\left(T_{X}\right)+n-3 .
$$

The variety $\bar{M}_{0, n}(X, \beta)$ is called the moduli space of stable maps, or the Kontsevich moduli space of stable maps of class $\beta$ from a rational pre-stable $n$-pointed curve to $X$.

Kontsevich spaces of conics in Grassmannians. We will denote by $\bar{M}_{0,0}(\mathbb{G}(k, n), 2)$ the moduli space of degree two stable maps to the Grassmannian $\mathbb{G}(k, n)$ parametrizing $k$-planes in $\mathbb{P}^{n}$ embedded via the Plücker embedding. Now, following [CC10, Section 2] we are going to describe divisor classes on $\bar{M}_{0,0}(\mathbb{G}(k, n), 2)$. Fix projective subspaces $\Pi^{n-k}, \Pi^{n-k-2} \subset \mathbb{P}^{n}$ of dimension $n-k$ and $n-k-2$, and consider the Schubert cycles

$$
\begin{aligned}
\sigma_{1,1}^{k, n} & =\left\{W \in \mathbb{G}(k, n) \mid \operatorname{dim}\left(W \cap \Pi^{n-k}\right) \geqslant 1\right\} ; \\
\sigma_{2}^{k, n} & =\left\{W \in \mathbb{G}(k, n) \mid \operatorname{dim}\left(W \cap \Pi^{n-k-2}\right) \geqslant 0\right\} .
\end{aligned}
$$

Let $\pi: \bar{M}_{0,1}(\mathbb{G}(k, n), 2) \rightarrow \bar{M}_{0,0}(\mathbb{G}(k, n), 2)$ be the forgetful morphism and $e v: \bar{M}_{0,1}(\mathbb{G}(k, n), 2) \rightarrow \mathbb{G}(k, n)$ the evaluation morphism. We define

$$
H_{\sigma_{1,1}}^{k, n}=\pi_{*} e v^{*} \sigma_{1,1}, H_{\sigma_{2}}^{k, n}=\pi_{*} e v^{*} \sigma_{2} .
$$

Furthermore, we will denote by $T^{k, n}$ the class of the divisor of conics that are tangent to a fixed hyperplane section of $\mathbb{G}(k, n)$.

Let $D_{d e g}^{k, n}$ be the class of the divisor of maps $[C, \alpha] \in \bar{M}_{0,0}(\mathbb{G}(k, n), 2)$ such that the projection of the span of the linear spaces parametrized by $\alpha(C)$ from a fixed subspace of dimension $n-k-2$ has dimension less than $k+2$.

Next we define the divisor class $D_{u n b}^{k, n}$. A stable map $\alpha: \mathbb{P}^{1} \rightarrow \mathbb{G}(k, n)$ induces a rank $k+1$ subbundle $\mathcal{E}_{\alpha} \subset \mathcal{O}_{\mathbb{P}^{1}} \otimes K^{n+1}$. If $k=1$ we define $D_{u n b}^{k, n}$ as the closure of the locus of maps $\left[\mathbb{P}^{1}, \alpha\right] \in \bar{M}_{0,0}(\mathbb{G}(k, n), 2)$ such that $\mathcal{E}_{\alpha} \neq \mathcal{O}_{\mathbb{P}^{1}}(-1)^{\oplus 2}$. If $k \geqslant 2$ there is a trivial subbundle $\mathcal{O}_{\mathbb{P}^{1}}^{\oplus k-1} \subset \mathcal{E}_{\alpha}$ which induces a $(k-2)$-dimensional subspace $H_{\alpha} \subset \mathbb{P}^{n}$. In this way we get a map

$$
\begin{array}{ccc}
\xi: \bar{M}_{0,0}(\mathbb{G}(k, n), 2) & \cdots & \mathbb{G}(k-2, n) \\
{\left[\mathbb{P}^{1}, \alpha\right]} & \mapsto & H_{\alpha}
\end{array}
$$

We define $D_{u n b}^{k, n}=\xi^{*} \mathcal{O}_{\mathbb{G}(k-2, n)}(1)$ that is $D_{u n b}^{k, n}$ is the closure of the locus of maps $\left[\mathbb{P}^{1}, \alpha\right] \in \bar{M}_{0,0}(\mathbb{G}(k, n), 2)$ such that $H_{\alpha}$ intersects a fixed $(n-k+1)$-dimensional subspace of $\mathbb{P}^{n}$.

Finally, we denote by $\Delta^{k, n}$ the boundary divisor parametrizing stable maps with reducible domain.

The connection between $\bar{M}_{0,0}(\mathbb{G}(1,3), 2)$ and the space of complete quadrics $\mathcal{Q}(3)$ is due to [Hue15, Lemma 21] which states that there is a finite morphism of degree two

$$
\phi: \bar{M}_{0,0}(\mathbb{G}(1,3), 2) \rightarrow \mathcal{Q}(3)
$$

which maps a smooth conic $C \subset \mathbb{G}(1,3)$ to the quadric surface $\bigcup_{[L] \in C} L \subset \mathbb{P}^{3}$. 
Kontsevich spaces of conics in Lagrangian Grassmannians. The Lagrangian Grassmannian $L G(r, 2 r) \subset \mathbb{G}(r-$ 1, $2 r-1)$ parametrizes $r$-dimensional subspaces of $K^{2 r}$ which are isotropic with respect to the standard symplectic form $\Omega$ in (3.1). By [Tev05, Section 2.1] $L G(r, 2 r)$ is an irreducible variety of dimension $\frac{r(r+1)}{2}$ and of Picard rank one. Moreover, the restriction of the Plücker embedding of $\mathbb{G}(r-1,2 r-1)$ yields the minimal homogeneous embedding of $L G(r, 2 r)$.

In this section we will study the moduli space $\bar{M}_{0,0}(L G(r, 2 r), 2)$ parametrizing conics in $L G(r, 2 r)$. Let $\mathcal{E}$ be the universal quotient bundle on $\mathbb{G}(r-1,2 r-1)$. The Lagrangian Grassmannian $L G(r, 2 r) \subset \mathbb{G}(r-1,2 r-1)$ is the zero locus of a section of $\bigwedge^{2} \mathcal{E}$ which has first Chern class $(r-1) c_{1}\left(\mathcal{O}_{\mathbb{G}(r-1,2 r-1)}(1)\right)$. Hence the canonical bundle of $L G(r, 2 r)$ is given by $\omega_{L G(r, 2 r)} \cong \mathcal{O}_{L G(r, 2 r)}(-r-1)$, and $\operatorname{dim}\left(\bar{M}_{0,0}(L G(r, 2 r), 2)\right)=\frac{r^{2}+5 r-2}{2}$.

Remark 6.2. We recall some facts about the cohomology of $L G(r, 2 r)$. For details we refer to BKT03, Section 3]. Consider a flag $F^{1} \subset F^{2} \subset \cdots \subset F^{r} \subset K^{2 r}$, where $F^{j}$ are isotropic subspaces of $K^{2 r}$ of dimension $j$. Let $\mathcal{D}_{r}$ be the set of strict partitions $\lambda=\left(\lambda_{1}, \ldots, \lambda_{l}\right)$ with $0<\lambda_{l}<\cdots<\lambda_{1} \leqslant r$ and denote by $|\lambda|=\lambda_{1}+\cdots+\lambda_{l}$ the weight of $\lambda$. For each $\lambda \in \mathcal{D}_{r}$ there is a codimension $|\lambda|$ Schubert variety $\Sigma_{\lambda}^{r} \subseteq L G(r, 2 r)$ defined by

$$
\Sigma_{\lambda}^{r}:=\left\{W \in L G(r, 2 r), \operatorname{dim}\left(W \cap F^{r+1-\lambda_{i}}\right) \geqslant i, i=1, \ldots, l\right\} .
$$

The class of the Schubert variety $\Sigma_{\lambda}^{r}$ in the cohomology ring $H^{*}(L G(r, 2 r), \mathbb{Z})$ will be denoted by $\sigma_{\lambda}^{r}$. We have that

$$
H^{*}(L G(r, 2 r), \mathbb{Z})=\bigoplus_{\lambda \in \mathcal{D}_{r}} \mathbb{Z} \cdot \sigma_{\lambda}^{r}
$$

with the following relations:

$$
\left(\sigma_{i}^{r}\right)^{2}+2 \sum_{k=1}^{r-i}(-1)^{k} \sigma_{i+k}^{r} \sigma_{i-k}^{r}=0
$$

where by convention $\sigma_{0}^{r}=1$ and $\sigma_{i}^{r}=0$ for $i<0$.

Now, we define divisor classes on $\bar{M}_{0,0}(L G(r, 2 r), 2)$. We denote by $\Delta^{r}$, the boundary divisor parametrizing stable maps with reducible domain, this is the restriction to $\bar{M}_{0,0}(L G(r, 2 r), 2)$ of the divisor $\Delta^{r-1,2 r-1}$ on $\bar{M}_{0,0}(\mathbb{G}(r-$ $1,2 r-1), 2)$.

Fix an isotropic subspace $F^{r-1}$ of dimension $r-1$, and consider the divisor $H_{\sigma_{2}}^{r}=\pi_{*} e v^{*} \sigma_{2}^{r}$, where $\pi$ : $\bar{M}_{0,1}(L G(r, 2 r), 2) \rightarrow \bar{M}_{0,0}(L G(r, 2 r), 2)$ is the forgetful morphism, ev : $\bar{M}_{0,1}(L G(r, 2 r), 2) \rightarrow L G(r, 2 r)$ is the evaluation morphism, and $\sigma_{2}^{r}$ is the Schubert cycle corresponding to the Schubert variety

$$
\Sigma_{2}^{r}:=\left\{W \in L G(r, 2 r), \operatorname{dim}\left(W \cap F^{r-1}\right) \geqslant 1\right\} .
$$

By Remark 6.2, in $L G(r, 2 r)$ the only Schubert cycle of codimension two is $\sigma_{2}^{r}$, so by Opr05, Theorem 1] we get that $\Delta^{r}$ and $H_{\sigma_{2}}^{r}$ generate the Picard group of $\bar{M}_{0,0}(L G(r, 2 r), 2)$. Furthermore, we have that both the divisors $H_{\sigma_{1,1}}^{r-1,2 r-1}$ and $H_{\sigma_{2}}^{r-1,2 r-1}$ of $\bar{M}_{0,0}(\mathbb{G}(r-1,2 r-1), 2)$ restrict to $H_{\sigma_{2}}^{r}$ on $\bar{M}_{0,0}(L G(r, 2 r), 2)$. Then, also $D_{d e g}^{r-1,2 r-1}$ and $D_{u n b}^{r-1,2 r-1}$ restrict to the same divisor $D_{u n b}^{r}$ on $\bar{M}_{0,0}(L G(r, 2 r), 2)$.

Finally, we will denote by $T^{r}$ the restriction of the divisor $T^{r-1,2 r-1}$ to $\bar{M}_{0,0}(L G(r, 2 r), 2)$, this is the class of the divisor of conics that are tangent to a fixed hyperplane section of $L G(r, 2 r)$.

Proposition 6.4. Consider the subspaces $H=\left\{x_{2}=\cdots=x_{r-1}=x_{r+2}=\cdots=x_{2 r-1}=0\right\}$ and $\Pi^{r-3}=\left\{x_{0}=\right.$ $\left.\cdots=x_{r+1}=0\right\}$ in $\mathbb{P}^{2 r-1}$. There is an embedding

$$
\begin{array}{ccc}
i: \quad L G(2, H) & \hookrightarrow & L G(r, 2 r) \\
L & \mapsto & \left\langle L, \Pi^{r-3}\right\rangle
\end{array}
$$

which induces an embedding $j: \bar{M}_{0,0}(L G(2,4), 2) \rightarrow \bar{M}_{0,0}(L G(r, 2 r), 2)$. Moreover, the pull-back map $j^{*}$ : $\operatorname{Pic}\left(\bar{M}_{0,0}(L G(r, 2 r), 2)\right) \rightarrow \operatorname{Pic}\left(\bar{M}_{0,0}(L G(2,4), 2)\right)$ is an isomorphism.

Proof. Since $\Pi^{r-3}$ is the projectivization of an isotropic subspace of $K^{2 r}$, and disjoint from $H$, the map is well-defined. By Opr05, Theorem 1] the Picard group of $\bar{M}_{0,0}(L G(r, 2 r), 2)$ is generated by $\Delta^{r}$ and $H_{\sigma_{2}}^{r}$.

Furthermore, we have that $i^{*}\left(\sigma_{2}^{r}\right)=\sigma_{2}^{2}$ and then $j^{*}\left(H_{\sigma_{2}^{r}}^{r}\right)=H_{\sigma_{2}^{2}}^{2}$. Finally, since $j^{*}\left(\Delta^{r}\right)=\Delta^{2}$ we conclude that the pull-back map is an isomorphism.

Lemma 6.5. Let $C_{1}, C_{2} \subset \mathbb{G}(1,3)$ be two smooth conics corresponding to the rulings $\bigcup_{[L] \in C_{1}} L$ and $\bigcup_{[L] \in C_{2}} L$ of a smooth quadric $Q \subset \mathbb{P}^{3}$. The following are equivalent:

(a) $C_{1}$ is contained in $L G(2,4)$ but $C_{2}$ is not;

(b) the lines in the ruling $\bigcup_{[L] \in C_{1}} L$ are Lagrangian while the general line in the ruling $\bigcup_{[L] \in C_{2}} L$ is not;

(c) the matrix of $Q$ has a scalar multiple that is symplectic. 
Proof. The actions of $S p(4)$ on $\bar{M}_{0,0}(L G(2,4), 2)$ in (6.7) and on $\mathcal{S}_{4}$ in (3.5) are compatible. Therefore, it is enough to prove that the equivalence of the conditions in statement holds for a particular smooth quadric.

Consider the quadric $Q=\left\{x_{0}^{2}+x_{1}^{2}-x_{2}^{2}-x_{3}^{2}=0\right\} \subset \mathbb{P}^{3}$. If $M_{Q}$ is the matrix of $Q$ we have $M_{Q}^{t} \Omega M_{Q}=-\Omega$, and hence $i M_{Q}$ is symplectic.

Now, one of the rulings of $Q$ is given by the following lines

$$
L_{s, t}=\langle(t,-s,-t, s),(s, t, s, t)\rangle
$$

with $[s: t] \in \mathbb{P}^{1}$. Note that $L_{s, t}$ is Lagrangian with respect to $\Omega$ for all $[s: t] \in \mathbb{P}^{1}$.

Fix homogeneous coordinates $\left[Z_{0}: \cdots: Z_{5}\right]$ on $\mathbb{P}^{5}$. The Lagrangian Grassmannian $L G(2,4)$ is cut out on the Grassmannian $\mathbb{G}(1,3)$ by the hyperplane $H=\left\{Z_{1}+Z_{4}=0\right\}$. Via the Plücker embedding the ruling $L_{s, t}$ corresponds to the conic given by the image of the following morphism

$$
\begin{aligned}
& \mathbb{P}^{1} \longrightarrow \mathbb{G}(1,3) \\
& (s, t) \longmapsto\left[t^{2}+s^{2}: 2 s t: t^{2}-s^{2}:-s^{2}+t^{2}:-2 s t:-t^{2}-s^{2}\right]
\end{aligned}
$$

which therefore is contained in $H \cap \mathbb{G}(1,3)=L G(2,4)$. The other ruling of $Q$ is given by

$$
R_{u, v}=\langle(u,-v, u, v),(v, u,-v, u)\rangle
$$

with $[u: v] \in \mathbb{P}^{1}$. The corresponding conic is given by the image of

$$
\begin{aligned}
& \mathbb{P}^{1} \longrightarrow \mathbb{G}(1,3) \\
& (u, v) \longmapsto\left[u^{2}+v^{2}:-2 u v: u^{2}-v^{2}: v^{2}-u^{2}:-2 u v, u^{2}+v^{2}\right]
\end{aligned}
$$

which is not contained in $H \cap \mathbb{G}(1,3)=L G(2,4)$. Hence, the general line in the ruling $R_{u, v}$ is not Lagrangian.

Lemma 6.6. The following $S p(4)$-action on $\bar{M}_{0,0}(L G(2,4), 2)$

$$
\begin{aligned}
& S p(4) \times \bar{M}_{0,0}(L G(2,4), 2) \quad \longrightarrow \quad \bar{M}_{0,0}(L G(2,4), 2) \\
& (M,[C, \alpha]) \quad \longmapsto\left[C, \wedge^{r} M \circ \alpha\right]
\end{aligned}
$$

gives to $\bar{M}_{0,0}(L G(2,4), 2)$ a structure of spherical variety.

Proof. By Lemma 6.5 a ruling of the quadric $Q=\left\{x_{0}^{2}+x_{1}^{2}-x_{2}^{2}-x_{3}^{2}=0\right\}$ yields a conic in $L G(2,4)$. Let $\mathscr{B} \subset S p(4)$ be the Borel subgroup of the symplectic group in $\operatorname{Remark}$ 3.4. Note that $\operatorname{dim}(\mathscr{B})=6$. The stabilizer of $Q$ in $\mathscr{B}$ is given by

$$
\left(\begin{array}{ll}
A_{2,2} & 0_{2,2} \\
B_{2,2} & A_{2,2}^{-t}
\end{array}\right)\left(\begin{array}{cc}
I_{2,2} & 0_{2,2} \\
0_{2,2} & -I_{2,2}
\end{array}\right)\left(\begin{array}{cc}
A_{2,2}^{t} & B_{2,2}^{t} \\
0_{2,2} & A_{2,2}^{-1}
\end{array}\right)=\left(\begin{array}{cc}
A_{2,2}^{t} A_{2,2} & A_{2,2} B_{2,2}^{t} \\
B_{2,2} A_{2,2}^{t} & B_{2,2} B_{2,2}^{t}-A_{2,2}^{-t} A_{2,2}^{-1}
\end{array}\right)
$$

So, we get $B_{2,2}=0_{2,2}$ and $A_{2,2}^{t} A_{2,2}=I_{2,2}$. Then

$$
\operatorname{Stab}_{\mathscr{B}}(Q)=\left\{M=\left(\begin{array}{cccc}
a & 0 & 0 & 0 \\
0 & b & 0 & 0 \\
0 & 0 & \frac{1}{a} & 0 \\
0 & 0 & 0 & \frac{1}{b}
\end{array}\right) ; \text { with } a^{2}=b^{2}=1\right\}
$$

and $\operatorname{dim}\left(\operatorname{Stab}_{\mathscr{B}}(Q)\right)=0$.

Proposition 6.8. The restriction of the map in (6.1) to $\bar{M}_{0,0}(L G(2,4), 2)$ yields an isomorphism

$$
\varphi: \bar{M}_{0,0}(L G(2,4), 2) \rightarrow \mathcal{S}_{4}
$$

where $\mathcal{S}_{4}$ is the wonderful compactification of the space of symplectic quadrics of $\mathbb{P}^{3}$.

Proof. By Lemma 6.5 the restriction of the map in (6.1) to $\bar{M}_{0,0}(L G(2,4), 2)$ yields a 1-to-1 morphism which is surjective since both $\bar{M}_{0,0}(L G(2,4), 2)$ and $\mathcal{S}_{4}$ are 6-dimensional.

Finally, since $\mathcal{S}_{4}$ is smooth and $\bar{M}_{0,0}(L G(2,4), 2)$ is normal Zariski's main theorem Mum99, Chapter 3, Section 9] yields that the morphism in (6.9) is an isomorphism.

Lemma 6.10. The divisor classes $\Delta^{2}, D_{u n b}^{2}$ and the divisor classes $H_{\sigma_{2}}^{2}, T^{2}$ are respectively the classes of the boundary divisors and the colors of the spherical variety $\bar{M}_{0,0}(L G(2,4), 2)$.

Proof. The actions (6.7) and (3.5) are equivariant with respect to the map $\varphi$ in (6.9). So boundary divisors and colors of $\bar{M}_{0,0}(L G(2,4), 2)$ are mapped by $\varphi$ to boundary divisors and colors of $\mathcal{S}_{4}$ respectively. By Proposition 4.6. in $\mathcal{S}_{4}$ the colors are $D_{1}, D_{2}$ and the boundary divisors are $E_{1}, S_{2}^{(1)}\left(\mathcal{V}_{2}^{3}\right)$. Moreover, $\Delta^{2}, D_{u n b}^{2}$ are stabilized by the $S p(4)$-action in (6.7) and choosing the flag of isotropic linear subspaces $\left\{x_{0}=x_{1}=0\right\} \subset\left\{x_{0}=0\right\}$ we see that $H_{\sigma_{2}}^{2}, T^{2}$ are stabilized by the action of the Borel subgroup of $S p(4)$ in Remark 3.4 Moreover, it is 
straightforward to see that the inverse image via the morphism $\varphi$ in (6.9) of $S_{2}^{(1)}\left(\mathcal{V}_{2}^{3}\right), E_{1}, D_{1}, D_{2}$ are divisors of classes $\Delta^{2}, D_{u n b}^{2}, H_{\sigma_{2}}^{2}, T^{2}$. Now, assume to have another boundary divisor in $\bar{M}_{0,0}(L G(2,4), 2)$. Then, $\varphi$ maps this divisor to a boundary divisor of $\mathcal{S}_{4}$, but the only boundary divisors of $\mathcal{S}_{4}$ are $S_{2}^{(1)}\left(\mathcal{V}_{2}^{3}\right), E_{1}$. Then, the only boundary divisors of $\bar{M}_{0,0}(L G(2,4), 2)$ are $\Delta^{2}, D_{u n b}^{2}$, and similarly the only colors of $\bar{M}_{0,0}(L G(2,4), 2)$ are $H_{\sigma_{2}}^{2}, T^{2}$.

We denote by $\bar{M}_{0,0}(L G(r, 2 r), 2,1)$ the moduli space of weighted stable maps to $L G(r, 2 r)$. In this space degree one tails of a stable map are replaced by their attaching point. We refer to [MM07] for the construction of moduli of weighted stable maps.

Proposition 6.11. The divisors $\Delta^{r}, D_{u n b}^{r}$ generate the effective cone of $\bar{M}_{0,0}(L G(r, 2 r), 2)$, and the divisors $H_{\sigma_{2}}^{r}, T^{r}$ generate the nef cone of $\bar{M}_{0,0}(L G(r, 2 r), 2)$.

The divisor $H_{\sigma_{2}}^{r}$ induces a birational morphism

$$
f_{H_{\sigma_{2}}^{r}}: \bar{M}_{0,0}(L G(r, 2 r), 2) \rightarrow \widetilde{\operatorname{Chow}}(L G(r, 2 r), 2)
$$

which is an isomorphism away form the locus $Q^{r}(1)$ of double covers of a line in $L G(r, 2 r)$, and contracts $Q^{r}(1)$ so that the locus of double covers with the same image maps to a point, where $\widetilde{C h o w}(L G(r, 2 r), 2)$ is the normalization of the Chow variety of conics in $L G(r, 2 r)$.

The divisor $T^{r}$ induces a morphism

$$
f_{T^{r}}: \bar{M}_{0,0}(L G(r, 2 r), 2) \rightarrow \bar{M}_{0,0}(L G(r, 2 r), 2,1)
$$

which is an isomorphism away from $\Delta^{r}$ and contracts the locus of maps with reducible domain $\left[C_{1} \cup C_{2}, \alpha\right]$ to $\alpha\left(C_{1} \cap C_{2}\right)$. Hence, $f_{T^{r}}$ contracts the divisor $\Delta^{r}$ onto $L G(r, 2 r) \subset \bar{M}_{0,0}(L G(r, 2 r), 2,1)$.

Proof. By [ADHL15, Proposition 4.5.4.4] and Lemma 6.10 the effective cone of $\bar{M}_{0,0}(L G(2,4), 2)$ is generated by $\Delta^{2}, D_{u n b}^{2}, H_{\sigma_{2}}^{2}, T^{2}$. Consider the isomorphism $\varphi$ in (6.9). We have

$$
\varphi^{*} E_{1}=D_{u n b}^{2}, \varphi^{*} S_{2}^{(1)}\left(\mathcal{V}_{2}^{3}\right)=\Delta^{2}, \varphi^{*} D_{1}=H_{\sigma_{2}}^{2}, \varphi^{*} D_{2}=T^{2} .
$$

Now, the relations among the boundary divisors and the colors of $\mathcal{S}_{4}$ in Proposition 5.10 yield the following relations in the Picard group of $\bar{M}_{0,0}(L G(2,4), 2)$ :

$$
H_{\sigma_{2}}^{2} \sim \frac{\Delta^{2}+2 D_{u n b}^{2}}{2}, T^{2} \sim \Delta^{2}+D_{u n b}^{2}
$$

and the statement in the case $r=2$ follows from Propositions 4.7 and 6.8 .

Now, consider the case $r>2$. Since $T^{r}$ is the pull-back of $T^{r-1,2 r-1}$ via the embedding $\bar{M}_{0,0}(L G(r, 2 r), 2) \hookrightarrow$ $\bar{M}_{0,0}(\mathbb{G}(r-1,2 r-1), 2)$ [CC10, Theorem 3.8] yields that $T^{r}$ induces a morphism

$$
f_{T^{r}}: \bar{M}_{0,0}(L G(r, 2 r), 2) \rightarrow \bar{M}_{0,0}(L G(r, 2 r), 2,1)
$$

which is an isomorphism away from $\Delta^{r}$ and contracts the locus of maps with reducible domain $\left[C_{1} \cup C_{2}, \alpha\right]$ to $\alpha\left(C_{1} \cap C_{2}\right)$. Hence, $f_{T^{r}}$ contracts the divisor $\Delta^{r}$ onto $L G(r, 2 r) \subset \bar{M}_{0,0}(L G(r, 2 r), 2,1)$. So $\Delta^{r}$ generates an extremal ray of the effective cone, and $T^{r}$ generates an extremal ray of the nef cone.

Similarly, [CC10, Proposition 3.7] yields the morphism $f_{H_{\sigma_{2}}^{r}}: \bar{M}_{0,0}(L G(r, 2 r), 2) \rightarrow \widetilde{\operatorname{Chow}}(L G(r, 2 r), 2)$, and hence $H_{\sigma_{2}}^{r}$ generates the other extremal ray of the nef cone.

Now, following the proof of [CC10, Lemma 3.4] we define the class of a curve $\Gamma$ in $\bar{M}_{0,0}(L G(r, 2 r), 2)$ whose deformations cover the whole of $\bar{M}_{0,0}(L G(r, 2 r), 2)$. Consider a general hyperplane section $Z$ of $L G(2,4) \subset \mathbb{P}^{4}$, and a general line in this hyperplane section. The planes containing the line cut out a pencil of conics on $Z \subset L G(2,4)$. Hence we get a rational curve $C \subset \bar{M}_{0,0}(L G(2,4), 2)$ parametrizing these conics. Let $\Gamma$ be the image of $C$ via the embedding in Proposition 6.4. Then $H_{\sigma_{2}}^{r} \cdot \Gamma=1$, and $\Delta^{r} \cdot \Gamma=2$ since there are two reducible conics in a general pencil of conics in the quadric surface $Z$. Now, by (6.12) we get that $D_{u n b}^{r} \cdot \Gamma=0$, and by [BDPP13, Theorem 2.2] we conclude that $D_{u n b}^{r}$ generates the other extremal ray of the effective cone.

Remark 6.13. Note that $Q^{r}(1)$ is a divisor in $\bar{M}_{0,0}(L G(r, 2 r), 2)$ if and only if $r=2$. By Proposition 6.8 we have $\bar{M}_{0,0}(L G(2,4), 2) \cong \mathcal{S}_{4}$ which by Proposition 3.16 is the blow-up of $\mathbb{G}(1,4)$ along the Veronese $\mathcal{V}_{2}^{3}$. In this case

$$
f_{H_{\sigma_{2}}^{2}}: \bar{M}_{0,0}(L G(2,4), 2) \rightarrow \widetilde{\operatorname{Chow}}(L G(2,4), 2)
$$

is nothing but the blow-down morphism $\mathcal{S}_{4} \rightarrow \mathbb{G}(1,4)$. Indeed, since $L G(2,4) \subset \mathbb{P}^{4}$ is a quadric hypersurface and hence does not contain any plane we have that all planes in $\mathbb{P}^{4}$ cut out a conic on $L G(2,4)$. Hence, we may identify the Chow variety of conics in $L G(2,4)$ with $\mathbb{G}(2,4) \cong \mathbb{G}(1,4)$. 
Furthermore, by Proposition 5.10 the morphism

$$
f_{T^{2}}: \bar{M}_{0,0}(L G(2,4), 2) \rightarrow \bar{M}_{0,0}(L G(2,4), 2,1)
$$

is induced by the strict transform of the restriction to $\mathbb{G}(1,4)$ of the linear system of quadrics in $\mathbb{P}^{9}$ containing $\mathcal{V}_{2}^{3}$. In this way we realize $\bar{M}_{0,0}(L G(2,4), 2,1)$ as a 6 -fold of degree 40 in $\mathbb{P}^{14}$ which is singular along a 3 -fold isomorphic to $L G(2,4)$.

Theorem 6.14. The Mori chamber decomposition of $\operatorname{Eff}\left(\bar{M}_{0,0}(L G(r, 2 r), 2)\right)$ has three chambers as displayed in the following picture:

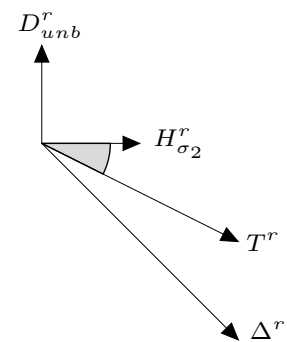

where $H_{\sigma_{2}}^{r} \sim \frac{1}{2}\left(\Delta^{r}+2 D_{\text {unb }}^{r}\right)$ and $T^{r} \sim \Delta^{r}+D_{u n b}^{r}$. Furthermore, $\operatorname{Mov}\left(\bar{M}_{0,0}(L G(r, 2 r), 2)\right)$ is generated by $T^{r}$ and $D_{u n b}^{r}$ if $r>2$, while $\operatorname{Mov}\left(\bar{M}_{0,0}(L G(2,4), 2)\right)$ is generated by $T^{2}$ and $H_{\sigma_{2}}^{2}$. The Cox ring $\operatorname{Cox}\left(\bar{M}_{0,0}(L G(2,4), 2)\right)$ is generated by the sections of $\Delta^{2}, D_{u n b}^{2}, H_{\sigma_{2}}^{2}, T^{2}$.

The birational model $X_{r}$ corresponding to the chamber delimited by $H_{\sigma_{2}}^{r}$ and $D_{u n b}^{r}$ is a fibration $X_{r} \rightarrow S G(r-$ $2,2 r)$ with fibers isomorphic to $\mathbb{G}(2,4)$, where $S G(r-2,2 r)$ is the symplectic Grassmannian parametrizing isotropic subspaces of dimension $r-2$. Finally, $D_{u n b}^{r}$ contracts $\bar{M}_{0,0}(L G(r, 2 r), 2)$ onto $S G(r-2,2 r)$.

Proof. First consider the case $r=2$. The statement on the generators of the Cox ring follows from Proposition 6.11 and Remark [5.6. Furthermore, by Remarks [5.5] and [5.6 the Mori chamber decomposition of $\operatorname{Eff}\left(\bar{M}_{0,0}(L G(2,4), 2)\right)$ is a, possibly trivial, coarsening of the decomposition in the statement. Since by Proposition 6.11 the effective cone $\operatorname{Eff}\left(\bar{M}_{0,0}(L G(2,4), 2)\right)$ is generated by $\Delta^{2}$ and $D_{u n b}^{2}$, and $H_{\sigma_{2}}^{2}, T^{2}$ generate $\operatorname{Nef}\left(\bar{M}_{0,0}(L G(2,4), 2)\right)$ no ray can be removed, and the Mori chamber decomposition is as in the statement. The relations $H_{\sigma_{2}}^{r} \sim \frac{1}{2}\left(\Delta^{r}+2 D_{u n b}^{r}\right)$ and $T^{r} \sim \Delta^{r}+D_{u n b}^{r}$ follow from the proof of Proposition 6.4 and (6.12).

Now, consider the case $r>2$. By Proposition 6.11 the wall-crossing of $T^{r}$ induces a divisorial contraction, and a divisor inside the chamber delimited by $T^{r}$ and $H_{\sigma_{2}}^{r}$ is ample. By Proposition 6.11 the wall-crossing of $H_{\sigma_{2}}^{r}$ yields a birational contraction whose exceptional locus is the variety $Q^{r}(1)$ of double covers of a line in $L G(r, 2 r)$.

Next, we will construct the birational model of $\bar{M}_{0,0}(L G(r, 2 r), 2)$ corresponding to the chamber delimited by $H_{\sigma_{2}}^{r}$ and $D_{u n b}^{r}$. Let $H \subset \mathbb{P}^{2 r-1}$ be an $(r+1)$-plane containing an isotropic $(r-1)$-plane $\Pi \subset \mathbb{P}^{2 r-1}$. Then $\Pi=\Pi^{\perp} \supset H^{\perp}$. So $H^{\perp} \subset H$. Now, the $(r+1)$-planes containing their orthogonal are in bijection with the $(r-3)$-planes of $\mathbb{P}^{2 r-1}$ that are isotropic. The variety parametrizing such $(r-3)$-planes is the symplectic Grassmannian $S G(r-2,2 r)$. Let $\mathcal{U}_{r}$ be the universal bundle on $S G(r-2,2 r), \mathcal{U}_{r}^{\perp} \subset \mathcal{U}_{r}$ its orthogonal, and $\mathcal{Q}_{r}=\mathcal{U}_{r} / \mathcal{U}_{r}^{\perp}$ the quotient bundle. Then $\mathcal{Q}_{r}$ has rank four, and we may consider the relative Lagrangian Grassmannian $L G\left(2, \mathcal{Q}_{r}\right) \rightarrow S G(r-2,2 r)$, and the relative Hilbert scheme $\operatorname{Hilb}_{2}\left(L G\left(2, \mathcal{Q}_{r}\right)\right) \rightarrow S G(r-2,2 r)$. Note that since $L G(2,4)$ does not contain planes the fibers of $\operatorname{Hilb}_{2}\left(L G\left(2, \mathcal{Q}_{r}\right)\right) \rightarrow S G(r-2,2 r)$ are isomorphic to $\mathbb{G}(2,4)$. Indeed, we can associate to a plane in $\mathbb{P}^{4}$ the conic it cuts out on $L G(2,4)$. Set $X_{r}:=\operatorname{Hilb}_{2}\left(L G\left(2, \mathcal{Q}_{r}\right)\right) \rightarrow S G(r-2,2 r)$. Note that

$$
\operatorname{dim}\left(X_{r}\right)=\operatorname{dim}(S G(r-2,2 r))+6=2 r^{2}-4 r-\frac{3(r-2)^{2}-r+2}{2}+6=\frac{r^{2}+5 r-2}{2}=\operatorname{dim}\left(\bar{M}_{0,0}(L G(r, 2 r), 2)\right)
$$

and there is a birational transformation $\bar{M}_{0,0}(L G(r, 2 r), 2) \rightarrow X_{r}$ inducing an isomorphism between the complement of $Q^{r}(1)$ in $\bar{M}_{0,0}(L G(r, 2 r), 2)$ and the complement of the locus of double lines in $X_{r}$. Since $r>2$ both these loci are in codimension greater that one. Furthermore, $H_{\sigma_{2}}^{r}$ induces a morphism on $X_{r}$ associating to a conic the reduced curve on which it is supported. Hence, this morphism is birational and contracts the locus of double lines. Finally $D_{u n b}^{r}$ induces on $X_{r}$ the fibration $X_{r} \rightarrow S G(r-2,2 r)$. Indeed, this fibration yields the rational fibration $\bar{M}_{0,0}(L G(r, 2 r), 2) \rightarrow S G(r-2,2 r)$ associating to a stable map that is not 2-to-1 onto a line the orthogonal of the $(r+1)$-plane in $\mathbb{P}^{2 r-1}$ generated by the $(r-1)$-planes parametrized by the image of the map. Hence, the cone generated by $H_{\sigma_{2}}^{r}$ and $D_{u n b}^{r}$ is the nef cone of $X_{r}$.

Finally, the claim about the movable cones follows from Remark 6.13 since $H_{\sigma_{2}}^{2}$ induces a divisorial contraction, while for $r>2$ the divisor $H_{\sigma_{2}}^{2}$ yields a small contraction and $D_{u n b}^{r}$ induces a non trivial fibration.

We now study the positivity of the anti-canonical divisor of $\bar{M}_{0,0}(L G(r, 2 r), 2)$. 
Proposition 6.15. Let $\overline{\mathcal{M}}_{0,0}(L G(r, 2 r), 2)$ be the smooth Deligne-Mumford stack of degree two stable maps to $L G(r, 2 r), \bar{H}_{\sigma_{2}}^{r}, \bar{T}^{r}, \bar{\Delta}^{r}, \bar{D}_{\text {unb }}^{r}$ the divisors on $\overline{\mathcal{M}}_{0,0}(L G(r, 2 r), 2)$ corresponding to $H_{\sigma_{2}}^{r}, T^{r}, \Delta^{r}, D_{\text {unb }}^{r}$ respectively.

The anti-canonical divisor of the stack $\overline{\mathcal{M}}_{0,0}(L G(r, 2 r), 2)$ is given by

$$
-K_{\overline{\mathcal{M}}_{0,0}(L G(r, 2 r), 2)}=5 \bar{H}_{\sigma_{2}}^{r}+\frac{r-7}{2} \bar{D}_{u n b}^{r}
$$

for $r>2$, while $-K_{\overline{\mathcal{M}}_{0,0}(L G(2,4), 2)}=5 \bar{H}_{\sigma_{2}}^{2}-5 \bar{D}_{\text {unb }}^{2}$. Furthermore, the anti-canonical divisor of $\bar{M}_{0,0}(L G(2,4), 2)$ is given by

$$
-K_{\bar{M}_{0,0}(L G(r, 2 r), 2)}=5 H_{\sigma_{2}}^{r}+\frac{r-7}{2} D_{u n b}^{r}
$$

for $r>2$, while for $r=2$ we have that

$$
-K_{\bar{M}_{0,0}(L G(2,4), 2)}=5 H_{\sigma_{2}}^{2}-2 D_{u n b}^{2} .
$$

Proof. We will compute the canonical divisor of $\overline{\mathcal{M}}_{0,0}(L G(r, 2 r), 2)$ using the formula in dJS17, Theorem 1.1]. Hence, we need the Chern classes $c_{1}\left(T_{L G(r, 2 r)}\right), c_{2}\left(T_{L G(r, 2 r)}\right)$, where $T_{L G(r, 2 r)}$ is the tangent bundle of $L G(r, 2 r)$. Recall that $T_{L G(r, 2 r)} \cong \operatorname{Sym}^{2}\left(S^{\vee}\right)$, where $S$ is the universal bundle.

Let us pretend that $S^{\vee}=L_{1} \oplus \cdots \oplus L_{r}$ splits as direct sum of line bundles. We will then use Whitney's formula along with the splitting principle to compute the Chern classes of $\operatorname{Sym}^{2}\left(S^{\vee}\right)$. Set $c_{1}\left(L_{i}\right)=\alpha_{i}$ for $i=1, \ldots, r$. Then

$$
c\left(S^{\vee}\right)=\prod_{i=1}^{r}\left(1+\alpha_{i}\right)
$$

and hence

$$
c_{1}\left(S^{\vee}\right)=\alpha_{1}+\cdots+\alpha_{r}, \quad c_{2}\left(S^{\vee}\right)=\alpha_{1} \alpha_{2}+\cdots+\alpha_{1} \alpha_{r}+\alpha_{2} \alpha_{3}+\cdots+\alpha_{r-1} \alpha_{r} .
$$

Furthermore

$$
\operatorname{Sym}^{2}\left(S^{\vee}\right)=L_{1}^{\otimes 2} \oplus\left(L_{1} \otimes L_{2}\right) \oplus \cdots \oplus\left(L_{1} \otimes L_{r}\right) \oplus L_{2}^{\otimes 2} \oplus \cdots \oplus L_{r}^{\otimes 2}
$$

yields

$$
\begin{aligned}
c\left(\operatorname{Sym}^{2}\left(S^{\vee}\right)\right)= & \left(1+2 \alpha_{1}\right)\left(1+\alpha_{1}+\alpha_{2}\right) \ldots\left(1+\alpha_{1}+\alpha_{r}\right)\left(1+2 \alpha_{2}\right) \ldots\left(1+2 \alpha_{r}\right)= \\
& 1+(r+1) \sum_{i=1}^{r} \alpha_{i}+\frac{r^{2}+r-2}{2} \sum_{i=1}^{r} \alpha_{i}^{2}+\left(r^{2}+2 r\right)\left(\alpha_{1} \alpha_{2}+\cdots+\alpha_{r-1} \alpha_{r}\right)+\cdots= \\
& 1+(r+1) \sum_{i=1}^{r} \alpha_{i}+\frac{r^{2}+r-2}{2}\left(\sum_{i=1}^{r} \alpha_{i}\right)^{2}+(r+2)\left(\alpha_{1} \alpha_{2}+\cdots+\alpha_{r-1} \alpha_{r}\right)+\cdots= \\
& 1+(r+1) c_{1}\left(S^{\vee}\right)+\frac{r^{2}+r-2}{2} c_{1}\left(S^{\vee}\right)^{2}+(r+2) c_{2}\left(S^{\vee}\right)+\ldots
\end{aligned}
$$

where in the last equality we plugged-in the formulas in (6.15). Recall that $c_{1}\left(S^{\vee}\right)=\sigma_{1}^{r}, c_{2}\left(S^{\vee}\right)=\sigma_{2}^{r}$ and that by (6.3) we have $\left(\sigma_{1}^{r}\right)^{2}=2 \sigma_{2}^{r}$. Hence

$$
c_{1}\left(T_{L G(r, 2 r)}\right)=(r+1) \sigma_{1}^{r}, \quad c_{2}\left(T_{L G(r, 2 r)}\right)=\left(r^{2}+2 r\right) \sigma_{2}^{r} .
$$

Now, plugging-in these formulas in dJS17, Theorem 1.1] we get

$$
K_{\overline{\mathcal{M}}_{0,0}(L G(r, 2 r), 2)}=-\frac{2 r+6}{4} \bar{H}_{\sigma_{2}}^{r}+\frac{r-7}{4} \bar{\Delta}^{r} .
$$

Let $\pi: \overline{\mathcal{M}}_{0,0}(L G(r, 2 r), 2) \rightarrow \bar{M}_{0,0}(L G(r, 2 r), 2)$ be the canonical morphism from $\overline{\mathcal{M}}_{0,0}(L G(r, 2 r), 2)$ to its coarse moduli space. Note that $\pi: \overline{\mathcal{M}}_{0,0}(L G(r, 2 r), 2) \rightarrow \bar{M}_{0,0}(L G(r, 2 r), 2)$ is an isomorphism in codimension one for all $r>2$, while for $r=2$ it is ramified on the divisor $D_{u n b}^{2}$. When $r=2$ the stack has non trivial inertia along the divisor $\bar{D}_{\text {unb }}^{2}$ since a general stable map in $\bar{D}_{\text {unb }}^{2}$ has automorphism group $\mathbb{Z} / 2 \mathbb{Z}$. Taking this into account we get that $\pi^{*} D_{u n b}^{2}=2 \bar{D}_{u n b}^{2}$, and hence Theorem 6.14 yields $\bar{\Delta}^{r}=2 \bar{H}_{\sigma_{2}}^{r}-2 \bar{D}_{u n b}^{r}$ if $r>2$, and $\bar{\Delta}^{2}=2 \bar{H}_{\sigma_{2}}^{2}-4 \bar{D}_{u n b}^{2}$. So, in terms of $\bar{H}_{\sigma_{2}}^{r}$ and $\bar{D}_{u n b}^{r}$ the canonical divisor of the stack is given by

$$
K_{\overline{\mathcal{M}}_{0,0}(L G(r, 2 r), 2)}=-5 \bar{H}_{\sigma_{2}}^{r}-\frac{r-7}{2} \bar{D}_{u n b}^{r}
$$

if $r>2$, and $K_{\overline{\mathcal{M}}_{0,0}(L G(2,4), 2)}=-5 \bar{H}_{\sigma_{2}}^{2}+5 \bar{D}_{u n b}^{2}$. Furthermore, when $r>2$ the formula above gives the expression of the canonical divisor of $\bar{M}_{0,0}(L G(r, 2 r), 2)$ in the statement since $\bar{M}_{0,0}(L G(r, 2 r), 2)$ and $\overline{\mathcal{M}}_{0,0}(L G(r, 2 r), 2)$ are isomorphic in codimension one for $r>2$.

However, when $r=2$ we have that

$$
K_{\overline{\mathcal{M}}_{0,0}(L G(2,4), 2)}=\pi^{*} K_{\bar{M}_{0,0}(L G(2,4), 2)}+\bar{D}_{u n b}^{2} .
$$


Let us write $K_{\bar{M}_{0,0}(L G(2,4), 2)}=-5 H_{\sigma_{2}}^{2}+a D_{u n b}^{2}$. Recalling that $\pi^{*} D_{u n b}^{2}=2 \bar{D}_{u n b}^{2}$ we get

$$
-5 \bar{H}_{\sigma_{2}}^{2}+5 \bar{D}_{u n b}^{2}=K_{\bar{M}_{0,0}(L G(2,4), 2)}=\pi^{*}\left(-5 H_{\sigma_{2}}^{2}+a D_{u n b}^{2}\right)+\bar{D}_{u n b}^{2}=-5 \bar{H}_{\sigma_{2}}^{2}+(2 a+1) \bar{D}_{u n b}^{2} .
$$

Hence, $a=2$ and $K_{\bar{M}_{0,0}(L G(2,4), 2)}=-5 H_{\sigma_{2}}^{2}+2 D_{u n b}^{2}$.

Remark 6.16. Since $\omega_{\mathbb{G}(1,4)}=\mathcal{O}_{\mathbb{G}(1,4)}(-5)$ and $\operatorname{codim}_{\mathbb{G}(1,4)}\left(\mathcal{V}_{2}^{3}\right)=3$ the formula $K_{\bar{M}_{0,0}(L G(2,4), 2)}=-5 H_{\sigma_{2}}^{2}+2 D_{u n b}^{2}$ can also be deduced from the description of $\bar{M}_{0,0}(L G(2,4), 2)$ as the blow-up of $\mathbb{G}(1,4)$ along $\mathcal{V}_{2}^{3}$ in Proposition 6.8 .

Corollary 6.17. The moduli space $\bar{M}_{0,0}(L G(r, 2 r), 2)$ is Fano for $2 \leqslant r \leqslant 6$, weak Fano for $r=7$, and $-K_{\bar{M}_{0,0}(L G(r, 2 r), 2)}$ is not ample for $r \geqslant 8$.

Proof. By Propositions 6.14 and 6.15 we have that $-K_{\bar{M}_{0,0}(L G(r, 2 r), 2)}$ is a multiple of $H_{\sigma_{2}}^{r}$ if $r=7$. Furthermore, $-K_{\bar{M}_{0,0}(L G(r, 2 r), 2)}$ lies in the interior of $\operatorname{Nef}\left(\bar{M}_{0,0}(L G(r, 2 r), 2)\right)$ for $2 \leqslant r \leqslant 6$, while for $r \geqslant 8$ we have that $-K_{\bar{M}_{0,0}(L G(r, 2 r), 2)}$ lies in the interior of the cone generated by $H_{\sigma_{2}}^{r}$ and $D_{u n b}^{r}$.

Finally, the following result on automorphisms of $\bar{M}_{0,0}(L G(2,4), 2)$ is at hand.

Corollary 6.18. The automorphism group of $\bar{M}_{0,0}(L G(2,4), 2)$ is given by

$$
\operatorname{PsAut}\left(\bar{M}_{0,0}(L G(2,4), 2)\right) \cong \operatorname{Aut}\left(\bar{M}_{0,0}(L G(2,4), 2)\right) \cong \operatorname{PSp}(4)
$$

where PSp(4) is the projective symplectic group, and $\operatorname{PsAut}\left(\bar{M}_{0,0}(L G(2,4), 2)\right)$ is the group of birational self-maps of $\bar{M}_{0,0}(L G(2,4), 2)$ inducing automorphisms in codimension one.

Proof. By Propositions $3.16,6.8$ we have that $\bar{M}_{0,0}(L G(2,4), 2)$ is isomorphic to the blow-up of $\mathbb{G}(1,4)$ along the Veronese $\mathcal{V}_{2}^{3}$. Let $\varphi \in \operatorname{Aut}\left(\bar{M}_{0,0}(L G(2,4), 2)\right)$ be an automorphism. Then either $\phi$ preserves the two extremal rays of Eff $\left(\bar{M}_{0,0}(L G(2,4), 2)\right)$ in Theorem 6.14] or it swaps them. In the second case $\phi$ must swap also the extremal rays of $\operatorname{Nef}\left(\bar{M}_{0,0}(L G(2,4), 2)\right)$ but this is not possible since for instance $T^{2}$ has more sections than $H_{\sigma_{2}}^{2}$. Therefore, $\phi$ stabilizes the exceptional divisor $D_{u n b}^{2}$ of the blow-up and then it induces an automorphism $\bar{\phi}$ of $\mathbb{G}(1,4)$ that stabilizes $\mathcal{V}_{2}^{3}$.

Now, the automorphism group of $\mathbb{G}(1,4)$ is isomorphic to $P G L(5)$ and all these automorphisms are induced by automorphisms of the ambient projective space $\mathbb{P}^{9}$ [Cow89, Theorem 1.1]. The restriction of $\bar{\phi}$ to $\mathcal{V}_{2}^{3}$ yields an automorphism $\bar{\phi}_{\mid \mathcal{V}_{2}^{3}}$ of $\mathbb{P}^{3}$. Since $\bar{\phi}$ is an automorphism of $\mathbb{G}(1,4)$, which we interpret as the closure of the space of symplectic and symmetric matrices modulo scalar, the restriction $\bar{\phi}_{\mid \mathcal{V}_{2}^{3}} \in P G L(4)$ must map symplectic matrices to symplectic matrices. Hence, $\bar{\phi}_{\mid \mathcal{V}_{2}^{3}} \in P S p(4)$. So, we get a morphism of groups

$$
\begin{aligned}
\chi: \operatorname{Aut}\left(\bar{M}_{0,0}(L G(2,4), 2)\right) & \rightarrow P S p(4) \\
\phi & \mapsto \bar{\phi}_{\mid \mathcal{V}_{2}^{3}}
\end{aligned}
$$

which is surjective. Now, if $\bar{\phi}_{\mid \mathcal{V}_{2}^{3}}$ is the identity it must be the restriction of the identity automorphism of the ambient projective space $\mathbb{P}^{9}$ in which both $\mathcal{V}_{2}^{3}$ and $\mathbb{G}(1,4)$ are embedded. Since $\mathbb{G}(1,4)$ and $\bar{M}_{0,0}(L G(2,4), 2)$ are birational we get that $\bar{\phi}_{\mid \mathcal{V}_{2}^{3}}$ must come from the identity of $\operatorname{Aut}\left(\bar{M}_{0,0}(L G(2,4), 2)\right)$, and hence $\chi$ is an isomorphism. Finally, since by Proposition 6.8 and Corollary $6.17 \bar{M}_{0,0}(L G(2,4), 2)$ is a smooth Fano variety the result on $\operatorname{PsAut}\left(\bar{M}_{0,0}(L G(2,4), 2)\right)$ follows from [Mas20a, Proposition 7.2].

\section{REFERENCES}

[AC17] C. Araujo and C .Casagrande, On the Fano variety of linear spaces contained in two odd-dimensional quadrics, Geom. Topol. 21 (2017), no. 5, 3009-3045. MR 3687113

[ADHL15] I. Arzhantsev, U. Derenthal, J. Hausen, and A. Laface, Cox rings, Cambridge Studies in Advanced Mathematics, vol. 144, Cambridge University Press, Cambridge, 2015. MR 3307753

[Alg56] A. R. Alguneid, Analytical degeneration of complete twisted cubics, Proc. Cambridge Philos. Soc. 52 (1956), 202-208. MR 0077998

[AM16] C. Araujo and A. Massarenti, Explicit log Fano structures on blow-ups of projective spaces, Proc. Lond. Math. Soc. (3) 113 (2016), no. 4, 445-473. MR 3556488

[BCHM10] C. Birkar, P. Cascini, C. D. Hacon, and J. McKernan, Existence of minimal models for varieties of log general type, J. Amer. Math. Soc. 23 (2010), no. 2, 405-468. MR 2601039

[BDPP13] S. Boucksom, J-P. Demailly, M. Păun, and T. Peternell, The pseudo-effective cone of a compact Kähler manifold and varieties of negative Kodaira dimension, J. Algebraic Geom. 22 (2013), no. 2, 201-248. MR 3019449

[BFS20] T. Brysiewicz, C. Fevola, and B. Sturmfels, Tangent Quadrics in Real 3-Space, https://arxiv.org/abs/2010.10879, 2020. 
[BKT03] A.S. Buch, A. Kresch, and H. Tamvakis, Gromov-Witten invariants on Grassmannians, J. Amer. Math. Soc. 16 (2003), no. 4, 901-915. MR 1992829

[BL11] P. Bravi and D. Luna, An introduction to wonderful varieties with many examples of type $\mathrm{F}_{4}$, J. Algebra 329 (2011), 4-51. MR 2769314

[BM21] M. Bolognesi and A. Massarenti, Birational geometry of moduli spaces of configurations of points on the line, Algebra \& Number Theory 15 (2021), no. 2, 513-544. MR 4243655

[Bos86] A. J. Bosch, The factorization of a square matrix into two symmetric matrices, Amer. Math. Monthly 93 (1986), no. 6, 462-464. MR 843191

[Bri89] M. Brion, Groupe de Picard et nombres caractéristiques des variétés sphériques, Duke Math. J. 58 (1989), no. 2, $397-424$. MR 1016427

[Bri93] _ Variétés sphériques et théorie de Mori, Duke Math. J. 72 (1993), no. 2, 369-404. MR 1248677

[Bri07] The total coordinate ring of a wonderful variety, J. Algebra 313 (2007), no. 1, 61-99. MR 2326138

[Bur65] M. Burrow, Representation theory of finite groups, Academic Press, New York-London, 1965. MR 0231924

[Cav16] F. Cavazzani, Complete homogeneous varieties via representation theory, Ph. D. Thesis, Harvard University, https://arxiv.org/abs/1603.09705 2016.

[CC10] D. Chen and I. Coskun, Stable base locus decompositions of Kontsevich moduli spaces, Michigan Math. J. 59 (2010), no. 2, 435-466. MR 2677631

[CC11] - Towards Mori's program for the moduli space of stable maps, Amer. J. Math. 133 (2011), no. 5, 1389-1419, With an appendix by Charley Crissman. MR 2843103

[Che08] D. Chen, Mori's program for the Kontsevich moduli space $\overline{\mathcal{M}}_{0,0}\left(\mathbb{P}^{3}, 3\right)$, Int. Math. Res. Not. IMRN (2008), Art. ID rnn 067, 17. MR 2439572

[CHS08] I. Coskun, J. Harris, and J. Starr, The effective cone of the Kontsevich moduli space, Canad. Math. Bull. 51 (2008), no. 4, 519-534. MR 2462457

[CHS09] _ The ample cone of the Kontsevich moduli space, Canad. J. Math. 61 (2009), no. 1, 109-123. MR 2488451

[CM17] K. Chung and H. B. Moon, Mori's program for the moduli space of conics in Grassmannian, Taiwanese J. Math. 21 (2017), no. 3, 621-652. MR 3661384

[Cow89] M. J. Cowen, Automorphisms of Grassmannians, Proc. Amer. Math. Soc. 106 (1989), no. 1, 99-106. MR 938909

[Cox95] D. A. Cox, The homogeneous coordinate ring of a toric variety, J. Algebraic Geom. 4 (1995), no. 1, 17-50. MR 1299003

[CS06] I. Coskun and J. Starr, Divisors on the space of maps to Grassmannians, Int. Math. Res. Not. (2006), Art. ID $35273,25$. MR 2264713

[CT06] A-M. Castravet and J. Tevelev, Hilbert's 14th problem and Cox rings, Compos. Math. 142 (2006), no. 6, $1479-1498$. MR 2278756

[DCP83] C. De Concini and C. Procesi, Complete symmetric varieties, Invariant theory (Montecatini, 1982), Lecture Notes in Math., vol. 996, Springer, Berlin, 1983, pp. 1-44. MR 718125

[Deb01] O. Debarre, Higher-dimensional algebraic geometry, Universitext, Springer New York, 2001.

[dJS17] A. J. de Jong and J. Starr, Divisor classes and the virtual canonical bundle for genus 0 maps, Geometry over nonclosed fields, Simons Symp., Springer, Cham, 2017, pp. 97-126. MR 3644251

[dlCb16] R. J. de la Cruz and H. Faßbender, On the diagonalizability of a matrix by a symplectic equivalence, similarity or congruence transformation, Linear Algebra Appl. 496 (2016), 288-306. MR 3464073

[FP97] W. Fulton and R. Pandharipande, Notes on stable maps and quantum cohomology, Algebraic geometry-Santa Cruz 1995, Proc. Sympos. Pure Math., vol. 62, Amer. Math. Soc., Providence, RI, 1997, pp. 45-96. MR 1492534

[Har95] J. Harris, Algebraic geometry, Graduate Texts in Mathematics, vol. 133, Springer-Verlag, New York, 1995, A first course, Corrected reprint of the 1992 original. MR 1416564

[HK00] Y. Hu and S. Keel, Mori dream spaces and GIT, Michigan Math. J. 48 (2000), 331-348, Dedicated to William Fulton on the occasion of his 60th birthday. MR 1786494

[HT84] J. Harris and L. W. Tu, On symmetric and skew-symmetric determinantal varieties, Topology 23 (1984), no. 1, 71-84. MR 721453

[Hue15] C. Lozano Huerta, Birational geometry of the space of complete quadrics, Int. Math. Res. Not. IMRN (2015), no. 23, 12563-12589. MR 3431630

[Kle80] S. L. Kleiman, Chasles's enumerative theory of conics: a historical introduction, Studies in algebraic geometry, MAA Stud. Math., vol. 20, Math. Assoc. America, Washington, D.C., 1980, pp. 117-138. MR 589410

[KP01] B. Kim and R. Pandharipande, The connectedness of the moduli space of maps to homogeneous spaces, Symplectic geometry and mirror symmetry (Seoul, 2000), World Sci. Publ., River Edge, NJ, 2001, pp. 187-201. MR 1882330

[KT88] S. Kleiman and A. Thorup, Complete bilinear forms, Algebraic geometry (Sundance, UT, 1986), Lecture Notes in Math., vol. 1311, Springer, Berlin, 1988, pp. 253-320. MR 951650

[Laz04] R. Lazarsfeld, Positivity in algebraic geometry. II, Ergebnisse der Mathematik und ihrer Grenzgebiete. 3. Folge. A Series of Modern Surveys in Mathematics [Results in Mathematics and Related Areas. 3rd Series. A Series of Modern Surveys in Mathematics], vol. 49, Springer-Verlag, Berlin, 2004, Positivity for vector bundles, and multiplier ideals. MR 2095472

[LLT89] D. Laksov, A. Lascoux, and A. Thorup, On Giambelli's theorem on complete correlations, Acta Math. 162 (1989), no. 3-4, 143-199. MR 989395

[LP17] J. Lesieutre and J. Park, Log Fano structures and Cox rings of blow-ups of products of projective spaces, Proc. Amer. Math. Soc. 145 (2017), no. 10, 4201-4209. MR 3690606

[Lun96] D. Luna, Toute variété magnifique est sphérique, Transform. Groups 1 (1996), no. 3, 249-258. MR 1417712

[Mas20a] A. Massarenti, On the birational geometry of spaces of complete forms I: collineations and quadrics, Proc. Lond. Math. Soc. (3) 121 (2020), no. 6, 1579-1618. MR 4144371

[Mas20b] _ On the birational geometry of spaces of complete forms II: Skew-forms, J. Algebra 546 (2020), 178-200. MR 4032731 
[MM07] Andrei Mustaţă and Magdalena Anca Mustaţă, Intermediate moduli spaces of stable maps, Invent. Math. 167 (2007), no. 1, 47-90. MR 2264804

[MP98] R. MacPherson and C. Procesi, Making conical compactifications wonderful, Selecta Math. (N.S.) 4 (1998), no. 1, 125-139. MR 1623714

[Muk01] S. Mukai, Counterexample to hilbert's fourteenth problem for the 3-dimensional additive group, Technical report, Kyoto University, Research Institute for Mathematical Sciences, 2001.

[Mum99] D. Mumford, The red book of varieties and schemes, expanded ed., Lecture Notes in Mathematics, vol. 1358, SpringerVerlag, Berlin, 1999, Includes the Michigan lectures (1974) on curves and their Jacobians, With contributions by Enrico Arbarello. MR 1748380

[Oka16] S. Okawa, On images of Mori dream spaces, Math. Ann. 364 (2016), no. 3-4, 1315-1342. MR 3466868

[Opr05] D. Oprea, Divisors on the moduli spaces of stable maps to flag varieties and reconstruction, J. Reine Angew. Math. 586 (2005), 169-205. MR 2180604

[OS94] G. Ottaviani and M. Szurek, On moduli of stable 2-bundles with small Chern classes on Q3, Ann. Mat. Pura Appl. (4) 167 (1994), 191-241, With an appendix by Nicolae Manolache. MR 1313556

[Ou12] S. Ou, Bijective maps on standard Borel subgroup of symplectic group preserving commutators, Front. Math. China 7 (2012), no. 3, 497-512. MR 2915792

[Per14] N. Perrin, On the geometry of spherical varieties, Transform. Groups 19 (2014), no. 1, 171-223. MR 3177371

[Pez18] G. Pezzini, Lectures on wonderful varieties, Acta Math. Sin. (Engl. Ser.) 34 (2018), no. 3, 417-438. MR 3763971

[Pie82] R. Piene, Degenerations of complete twisted cubics, Enumerative geometry and classical algebraic geometry (Nice, 1981), Progr. Math., vol. 24, Birkhäuser, Boston, Mass., 1982, pp. 37-50. MR 685763

[Sch10] H. Schoutens, The use of ultraproducts in commutative algebra, Lecture Notes in Mathematics, vol. 1999, Springer-Verlag, Berlin, 2010. MR 2676525

[Sem48] J. G. Semple, On complete quadrics, J. London Math. Soc. 23 (1948), 258-267. MR 0028605

[Sem51] _ The variety whose points represent complete collineations of $S_{r}$ on $S_{r}^{\prime}$, Univ. Roma. Ist. Naz. Alta Mat. Rend. Mat. e Appl. (5) 10 (1951), 201-208. MR 0048847

[Sem52] - On complete quadrics. II, J. London Math. Soc. 27 (1952), 280-287. MR 0048846

[Tev05] E. A. Tevelev, Projective duality and homogeneous spaces, Encyclopaedia of Mathematical Sciences, vol. 133, SpringerVerlag, Berlin, 2005, Invariant Theory and Algebraic Transformation Groups, IV. MR 2113135

[Tha99] M. Thaddeus, Complete collineations revisited, Math. Ann. 315 (1999), no. 3, 469-495. MR 1725990

[Tyr56] J. A. Tyrrell, Complete quadrics and collineations in $S_{n}$, Mathematika 3 (1956), 69-79. MR 0080352

[Vai82] I. Vainsencher, Schubert calculus for complete quadrics, Enumerative geometry and classical algebraic geometry (Nice, 1981), Progr. Math., vol. 24, Birkhäuser, Boston, Mass., 1982, pp. 199-235. MR 685770

[Vai84] Complete collineations and blowing up determinantal ideals, Math. Ann. 267 (1984), no. 3, 417-432. MR 738261

[Was96] B. Wasserman, Wonderful varieties of rank two, Transform. Groups 1 (1996), no. 4, 375-403. MR 1424449

Elsa Corniani, Dipartimento di Matematica e Informatica, Università di Ferrara, Via Machiavelli 30,44121 FerRARA, ITALY

Email address: elsa.corniani@unife.it

Alex Massarenti, Dipartimento di Matematica e Informatica, Università di Ferrara, Via Machiavelli 30,44121 Ferrara, Italy

Email address: alex.massarenti@unife.it 